\title{
Integrabilidade do Fluxo Geodésico na Solução de Kerr
}

\author{
Mário Otávio Salles
}

DISSERTAÇÃO APRESENTADA

$\mathrm{AO}$

INSTITUTO DE MATEMÁTICA E ESTATÍSTICA

DA

UNIVERSIDADE DE SÃO PAULO

PARA

OBTENÇÃO DO GRAU DE MESTRE

EM

MATEMÁTICA APLICADA

\begin{abstract}
Área de Concentração: Matemática Aplicada Orientador : Prof. Dr. Frank Michael Forger
\end{abstract}

Durante a elaboração deste trabalho o autor recebeu apoio financeiro do $C N P q$

- São Paulo, 25 de março de 1999 - 


\section{Integrabilidade \\ do Fluxo Geodésico na \\ Solução de Kerr}

Este exemplar corresponde à redação final da dissertação devidamente corrigida e defendida por Mário Otávio Salles e aprovada pela comissão julgadora.

São Paulo, 24 de março de 1999.

Banca examinadora:

- Prof. Dr. Frank Michael Forger (Orientador) - IME - USP

- Prof. Dr. Paolo Piccione - IME - USP

- Prof. Dr. Élcio Abdalla-IF- USP 
à Sabrina,

por todos estes anos, onde trilhamos o mesmo caminho,

ao meu pai Mário,

por tudo que ele significou, um pai. 
Omnia Mecum Porto

"Levo comigo tudo que tenho." Bias - 590 A.C.

Um dos Sete Sábios Gregos. 


\section{Agradecimentos}

Gostaria de deixar aqui os nomes daquelas pessoas que estiveram presentes nestes anos na USP ou que então torceram pela realização deste trabalho.

À Sabrina. pela leitura e sugestões que tornaram este trabalho mais interessante e também pelo estímulo e entusiasmo, que foram essenciais para vencer mais esta etapa.

Ao meu pai .Yário, que não está mais aqui, por todos os momentos felizes.

À minha màe Anne, que me deu a difícil liberdade de buscar o meu mundo e pela compreensão e apoio, mesmo que velado, na escolha das minhas opções.

À toda a minha família, que lá do Sul sempre estiveram torcendo por mim, em especial minha tia Altanisa e meus irmãos Diogo e Cristiano. À minha família adotiva Sérgio. Marion, Eduardo e André por terem me acolhido e tornado assim estes anos em São Paulo muito especiais.

Aos Mestres Alcides de Lima e Dorival dos Santos (CEACA) por terem me ensinado esta arte chamada Capoeira, e que neste mundo, sempre é o Rio que corre pro Mar.

Ao grupo do CEOP - Centro de Educação e Organização Popular - pela força e pela coragem de dizer sim mesmo quando todos diziam nào.

A todos meus professores da pós-graduação, que são os reponsáveis pela minha formaçào em Matemática. em especial à Profa. Elza.

Agradeço aos colegas Fernando e Sandro, pela atenção e pela paciência em me auxiliar a reconhecer os diferentes aspectos envolvidos nesta tese e principalmente nos meus primeiros passos nesta ciência, a Matemática.

Aos amigos Albetã, Rogério, Claudio e Beto, pois fizemos os nossos anos de CRLSP inesquecíveis e aos colegas do IME, Alice, Carlos, Gaspar, Germán, Glaúcio, Jocirei, Jorge, Leandro, Sandra e Santos, pelas discussões que sempre foram agradáveis e interessantes.

Finalmente. um agradecimento especial, merece o Prof. Michael Forger, por todos estes anos de convívio. tanto na graduação quanto no mestrado, pela importante experiência adquirida e pela excelente orientação. Além disso gostaria de agradecer a amizade e a paciência com que aceitou minhas dificuldades. 


\section{Resumo}

Neste trabalho, provamos a integrabilidade do fluxo geodésico na solução de Kerr, que inclui como caso especial a soluçào de Schwarzschild e descreve a geometria do espaço-tempo ao redor de um corpo isolado em rotação, em particular uma estrela de neutrons (pulsar) ou um buraco negro em rotação. Para verificar a integrabilidade do fluxo geodésico em uma variedade de Einstein, são necessárias quatro constantes do movimento que comutam. Porém, o fluxo geodésico para a solução de Kerr apresenta apenas três constantes de movimento que comutam e são mais ou menos óbvias: a Hamiltoniana $H$, a energia $E$ e o momento angular $L_{3}$ em torno do eixo de rotaçào. Uma quarta quantidade conservada foi descoberta por Carter e hoje é conhecida como constante de Carter: como a Hamiltoniana $H$, ela é quadrática nos momentos. Sua existência decorre do fato de que a métrica de Kerr admite um segundo tensor de Killing de posto 2, além do tensor métrico, que foi encontrado pela primeira vez por Walker e Penrose. Na presente dissertação, mostramos que estes dois tensores de Killing e os dois vetores de Killing tradicionais comutam sob o colchete de Schouten e que. portanto, as quatro constantes de movimento do fluxo geodésico no espaço-tempo de lierr estão em involução. Ademais, mostramos que estas constantes também são funcionalmente independentes, o que permite concluir que o fluxo geodésico no espaço-tempo de Kerr é um sistema Hamiltoniano completamente integrável, no sentido de Liouville.

\section{Abstract}

In this work, we prove integrability of the geodesic flow in the Kerr solution, which includes as a special case the Schwarzschild solution and describes the geometry of space-time outside an isolated rotating body, in particular a rotating neutron star (pulsar) or black hole. To verify integrability of the geodesic flow in an Einstein manifold, one must exhibit four commuting constants of motion. However, the geodesic flow for the Kerr solution only presents three commuting constants of motion which are more or less obvious: the Hamiltonian $H$, the energy $E$ and the angular momentum $L_{3}$ along the rotation axis. A fourth conserved quantity was discovered by Carter and is nowadays known as Carter's constant: just like the Hamiltonian $H$, it is quadratic in momenta. Its existence is due to the fact that the Kerr metric admits a second rank 2 Killing tensor, besides the metric tensor, which was first found by Walker and Penrose. In the present dissertation, we show that these two Killing tensors and the standard two Killing vectors commute under the Schouten bracket and that, therefore, the four constants of motion of the geodesic flux in Kerr space-time are in involution. Moreover, we show that these constants are also functionally independent, which allows to conclude that the geodesic flow in Kerr space-time is a completely integrable Hamiltonian system, in the sense of Liouville. 


\section{Conteúdo}

Introdução 2

1 Mecânica Geométrica $\quad 9$

1.1 Formalismo Hamiltoniano . . . . . . . . . . . . . . . . . . . . . . 10

1.2 Simetrias e Leis de Conservação: Aplicação Momento . . . . . . . . . . . . . 15

1.3 Formalismo Lagrangiano . . . . . . . . . . . . . . . . . . . . . 20

2 Fluxo Geodésico $\quad 24$

2.1 Geometria pseudo-Riemanniana . . . . . . . . . . . . . . . . . 24

2.2 Fluxo Geodésico . . . . . . . . . . . . . . . . . . . 26

2.3 Isometrias e Campos Vetoriais de Killing . . . . . . . . . . . . . . . . . 28

2.4 Campos Tensoriais de Killing . . . . . . . . . . . . . . . . . . 31

3 Relatividade Geral $\quad 34$

3.1 Soluções Exatas . . . . . . . . . . . . . . . . . . . . . . . 35

3.2 Solução de Schwarzschild . . . . . . . . . . . . . . . . . . . 37

3.3 Solução de Kierr . . . . . . . . . . . . . . . . . . . . . . . . . . . . 42

$\begin{array}{ll}\text { A Colchete de Schouten } & 56\end{array}$

$\begin{array}{ll}\text { B Formas de Conexão } & 60\end{array}$

$\begin{array}{ll}\text { C O Tensor de Killing } & 67\end{array}$ 


\section{Introdução}

A noção de um sistema integrável é de importância central na mecânica, na teoria dos campos e na teoria dos sistemas dinâmicos, inclusive pelo fato de que as equações de evolução de um sistema integrável são exatamente solúveis e, reciprocamente, todas as equações de evolução que admitem uma solução exata geral (i.e., para condições iniciais gerais) podem ser associadas com algum sistema integrável. Exemplos bem conhecidos na mecânica sào: a partícula livre, o oscilador harmônico, o problema de Kiepler, o corpo rígido, entre outros.

No contexto de sistemas Hamiltonianos com um número finito de graus de liberdade, uma definição geral de que seria um sistema integrável foi dada por Liouville. ${ }^{1}$ Ela se baseia na observação que, dado um sistema Hamiltoniano com Hamiltoniana $H$ e espaço de fase $M$, de dimensão $2 n$, que admite uma constante do movimento $F$, considerada como função $F: M \rightarrow \mathbb{R}$, então o teorema do fluxo tubular garante que para qualquer ponto $m_{0}$ de $M$ onde a diferencial de $F$ não se anula $\left(d F\left(m_{0}\right) \neq 0\right)$, existe um sistema de coordenadas canônicas $\left(x_{1}, \ldots, x_{n}, y_{1}, \ldots, y_{n}\right)$ em torno deste ponto tal que $F=y_{1}$. Nestas coordenadas, a Hamiltoniana $H$ nào depende de $x_{1}$ e portanto, as equações de Hamilton

$$
\dot{x}_{k}=\frac{\partial H}{\partial y_{k}} \quad, \quad \dot{y}_{k}=-\frac{\partial H}{\partial x_{k}} \quad(k=1, \ldots, n)
$$

do sistema original, $\operatorname{com} n$ graus de liberdade, se reduz a uma família de equações de Hamilton

$$
\dot{x}_{k}=\frac{\partial H}{\partial y_{k}} \quad, \quad \dot{y}_{k}=-\frac{\partial H}{\partial x_{k}} \quad(k=2, \ldots, n)
$$

com $n-1$ graus de liberdade e $y_{1}$ como parâmetro. Assim, a existência de uma constante do movimento, i.e., uma função $F$ que é constante ao longo das trajetórias do campo Hamiltoniano $X_{H}$, nos permite reduzir em dois graus a dimensão do espaço de fase (e não em um, como no caso de sistemas não Hamiltonianos): um decréscimo é obtido fixando o valor de $F=y_{1}$, pelo princípio acima, e o segundo, eliminando a variável cíclica conjugada $x_{1}$.

Este procedimento pode ser repetido, levando à conclusào que se um sistema Hamiltoniano tiver $k$ constantes do movimento $F_{1}, \ldots, F_{k}$ que estào em involuçào, i.e., cujos colchetes

\footnotetext{
${ }^{1}$ Neste trabalho, não consideraremos sistemas Hamiltonianos com um número infinito de graus de liberdade, para os quais existem várias possibilidades de definir conceitos de integrabilidade, que não são equivalentes.
} 
de Poisson se anulam, entào em torno de qualquer ponto de $M$ onde as diferenciais de $F_{1}, \ldots, F_{k}$ são linearmente independentes, o sistema pode ser reduzido a uma família de sistemas com $n-k$ graus de liberdade, parametrizada por $F_{1}, \ldots, F_{k}$. Finalmente, se este processo de redução pode ser repetido $k=n$ vezes, então segundo a definição de Liouville, o sistema é chamado completamente integrável.

A noção de um sistema integrável, apesar de originalmente ter sido desenvolvido e aplicado no âmbito da mecânica não-relativística de Newton, também se mostra útil para a mecânica relativística de Einstein, particularmente no caso da Relatividade Geral. Nesta, o movimento retilíneo uniforme de uma "partícula livre" da mecânica não-relativística é substituído pelo movimento de uma "partícula em queda livre" (o que inclui um raio de luz, visto como partícula com massa de repouso $m$ igual a zero - o fóton), e esse movimento é matematicamente descrito pelo fluxo geodésico na variedade Lorentziana usada como modelo do espaço-tempo.

Por outro lado, o fluxo geodésico em variedades Riemannianas ocupa uma posição de destaque na mecânica não-relativística. Do ponto de vista teórico, é interessante pois caracteriza a maneira mais geral de definir um sistema Hamiltoniano com energia puramente cinética, i.e., que seja uma forma quadrática nos momentos: esta forma quadrática é exatamente o (inverso do) tensor métrico, não havendo contribuições lineares nos momentos, como aconteceria na presença de forças eletromagnéticas (potenciais de calibre), ou contribuições independentes dos momentos. como aconteceria na presença de forças conservativas (energia potencial). Do ponto de vista prático é interessante, pois existem exemplos muito importantes, tais como as equações de Euler para o corpo rígido, que são exatamente as equações de movimento do fluxo geodésico sobre o grupo de rotações $S O(3)$ [1, pp. 311-337].

Portanto, uma questão natural é sob quais condições o fluxo geodésico numa dada variedade pseudo-Riemanniana constitui um sistema completamente integrável. Obviamente, isso ocorre quando o tensor de curvatura se anula, pois neste caso, existem sistemas de coordenadas locais $q^{i}$ nos quais os componentes do tensor métrico são constantes, e basta escolher $F_{i}=p_{i}(i=1, \ldots, n)$. Desta forma, recuperamos o movimento retilíneo uniforme da mecânica nào-relativística - o mais simples de todos os sistemas integráveis.

Nesta dissertação, demonstraremos a integrabilidade do fluxo geodésico em uma variedade Lorentziana específica conhecida na Relatividade Geral como a solução de Kerr - uma generalização da célebre solução de Schwarzschild - que descreve o campo gravitacional gerado por um corpo isolado em repouso e sem movimentos internos, mas girando em torno de um eixo fixo com velocidade angular constante. (A palavra "solução" refere-se ao fato de que a métrica de Kerr, assim como a métrica de Schwarzschild, satisfaz as equações de Einstein no vácuo,

$$
R_{\mu \nu}-\frac{1}{2} R g_{\mu \nu}=0,
$$

onde $R_{\mu \nu}$ é o tensor de Ricci associado ao tensor métrico $g_{\mu \nu}$; métricas/variedades com esta propriedade sào chamadas de métricas/variedades de Einstein.) O resultado correspondente (i.e., a integrabilidade do fluxo geodésico) para a soluçào de Schwarzschild - apesar de 
raramente ser formulado nestes termos - é bem conhecido, sendo que o comportamento de geodésicas na solução de S'chwarzschild é estudado em todos os livros texto sobre Relatividade Geral e é utilizado para deduzir as previsões mais tradicionais e espetaculares desta teoria: a rotação do periélio de Mercúrio e a defleçào de raios de luz no campo gravitacional do sol. Cabe salientar, porém, que - ao contrário da teoria Newtoniana de gravitaçào - a Relatividade Geral prevê que o campo gravitacional de um corpo com movimento rotacional é diferente do campo gravitacional de um corpo sem movimento rotacional. Esta diferença se manifesta no efeito do "arrastamento dos sistemas de referência", devido as forças giroscópicas [10], teoricamente predito por Lense e Thirring em 1918 e experimentalmente confirmado na observação dos buracos negros existentes nos centros das galáxias GRO J1655 - 40 e GRS 1915 + 105, que além de sugar a matéria de estrelas próximas, "arrastam" o próprio espaço-tempo ao seu redor [21, 22]. Portanto, de forma geral, a importância da solução de Kierr para a astronomia é indiscutível. uma vez que praticamente todos os corpos estelares apresentam algum movimento rotacional, inclusive os que geram campos gravitacionais fortes, como os pulsares e os buracos negros. Em particular, o estudo do comportamento de geodésicas na solução de Kerr constitui um tópico de grande importância para a astronomia, que infelizmente é quase completamente negligenciado nos livros texto sobre Relatividade Geral.

A principal contribuição à demonstração da integrabilidade do fluxo geodésico na solução de Kerr é devida a Carter [2, 3]. Para provar a integrabilidade do fluxo geodésico em uma variedade de Einstein, são necessárias quatro constantes do movimento, enquanto que no caso da métrica de Kerr, há apenas três constantes do movimento óbvias: a Hamiltoniana $H$, a energia $E$ e o momento angular $L_{3}$ em torno do eixo de rotação. No entanto, estudando as equações de Hamilton-Jacobi para o movimento geodésico [2] na métrica de Kerr, que devido à conservação da energia e do momento angular pode ser reduzida a uma equação diferencial parcial em duas variáveis. Carter descobriu uma quarta constante do movimento, hoje chamada a constante de Carter, que permite a solução desta equação por separação de variáveis, ou seja, sua redução a um sistema de duas equações diferenciais ordinárias. Posteriormente, Carter obteve o mesmo resultado para a equação de Klein-Gordon, que descreve a propagação de um campo escalar [3].

O próximo passo importante foi a introdução da noção de um tensor de Killing (ou mais exatamente, campo tensorial de Killing) que generaliza a noção clássica de um vetor de Killing (ou mais exatamente, campo vetorial de Killing), no sentido de providenciar constantes de movimento para o fluxo geodésico, porém sem a mesma interpretação geométrica de um gerador de um grupo a um parâmetro de isometrias. Em qualquer variedade pseudoRiemanniana, sempre existe pelo menos um tensor de Killing de ordem 2, que é o próprio tensor métrico, sendo que a constante de movimento associada é simplesmente a Hamiltoniana $H$ do fluxo geodésico. O primeiro exemplo não-trivial deste novo conceito, no entanto, parece ter originado com a observação de Walker e Penrose [20] de que a existência da constante de Carter decorre do fato de que a métrica de Fierr admite um segundo tensor de Killing $\kappa$. também de ordem 2 mas diferente do tensor métrico, que chamaremos aqui de tensor de 
Walker-Penrose. Este tensor é construído usando os dois campos vetoriais nulos principais da métrica de Kerr, cujas curvas integrais representam raios de luz que propagam radialmente com respeito ao centro de atração: portanto, estes campos vetoriais $N_{+}$e $N_{-}$devem ser nulos (tipo luz) e autoparalelos, pois isso garante que suas curvas integrais são geodésicas tipo luz. Explicitamente, em termos de coordenadas de Boyer-Lindquist $(t, r, \vartheta, \varphi)$, onde o tensor métrico é dado por

$$
\begin{aligned}
d s^{2}= & -\left(1-\frac{2 m r}{\rho^{2}}\right) d t^{2}+\frac{\rho^{2}}{\Delta} d r^{2}+\rho^{2} d \vartheta^{2} \\
& +\left(r^{2}+a^{2}+\frac{2 m a^{2} r \sin ^{2} \vartheta}{\rho^{2}}\right) \sin ^{2} \vartheta d \varphi^{2}-\frac{2 m a r \sin ^{2} \vartheta}{\rho^{2}} 2 d t d \varphi
\end{aligned}
$$

com

$$
\begin{aligned}
& \rho^{2}=r^{2}+a^{2} \cos ^{2} \vartheta, \\
& \Delta=r^{2}-2 m r+a^{2},
\end{aligned}
$$

e onde $m$ e $a$ são parâmetros reais $(m>0)$ representando, respectivamente, a massa e o momento angular do corpo central que gera o campo gravitacional descrito por esta métrica, temos

$$
N_{ \pm}= \pm \partial_{r}+\frac{r^{2}+a^{2}}{\Delta} \partial_{t}+\frac{a}{\Delta} \partial_{\varphi}
$$

$\mathrm{e}$

$$
K=\frac{\Delta}{2}\left(N_{+} \odot N_{-}+N_{-} \odot N_{+}\right)+r^{2} g
$$

O passo final consiste em verificar que as quatro constantes de movimento assim obtidas, consideradas como funções sobre o espaço de fase para o fluxo geodésico, que é o fibrado cotangente do espaço-tempo, comutam sob o colchete de Poisson e são funcionalmente independentes. A última afirmação pode ser verificado por um cálculo direto, enquanto que a demonstração da primeira pode ser substancialmente simplificada através de uma análise mais aprofundada da relação entre tensores de Killing e as constantes de movimento associadas, usando como conceito central o colchete de Schouten entre campos multivetoriais simétricos [18]:

- Por definição, um campo multivetorial simétrico de ordem $k$ sobre uma variedade $Q$ é simplesmente um campo tensorial totalmente contravariante e simétrico de ordem $k$ sobre $Q$. Se $K_{1}$ e $K_{2}$ são dois campos multivetoriais simétricos de ordem $k_{1}$ e de ordem $k_{2}$, respectivamente, então seu colchete de Schouten é um campo multivetorial simétrico de ordem $k_{1}+k_{2}-1$ sobre $Q$ que costuma ser denotado por $\left[K_{1}, K_{2}\right]$. Este colchete de Schouten é uma generalizaçào do colchete de Lie entre campos vetoriais e da derivada de Lie de campos multivetoriais, i.e., para $k_{1}=k_{2}=1,\left[K_{1}, K_{2}\right]$ coincide com o colchete de Lie dos campos vetoriais $K_{1}$ e $K_{2}$, enquanto que, mais geralmente, para $k_{1}=1\left(k_{2}=1\right),\left[K_{1}, K_{2}\right]$ coincide com a derivada de Lie de $K_{2}$ ao longo de $K_{1}$ (menos a derivada de Lie de $K_{1}$ ao longo de $K_{2}$ ). Ademais. o colchete de Schouten é bilinear (sobre $\mathbb{R}$ ), antisimétrico e satisfaz a identidade de Jacobi. 
- Dado um campo multivetorial simétrico $K$ de ordem $k$ sobre uma variedade pseudoRiemanniana $Q$ qualquer, $K$ é um campo tensorial de Killing se e somente se seu colchete de Schouten com o (inverso do) tensor métrico g se anula:

$$
[K, \mathrm{~g}]=0 .
$$

De fato, como no caso $k=1$, é fácil mostrar que esta condição é equivalente à condiçào de que $K$ satisfaça a equação de Killing

$$
\nabla_{(\mu} K_{\left.\mu_{1} \ldots \mu_{k}\right)}=0,
$$

onde $\nabla$ é a conexão de Levi-Civita e os parênteses indicam simetrização total.

- Dado um campo multivetorial simétrico $K$ de ordem $k$ sobre uma variedade $Q$, podemos associar a $K^{\prime}$ uma funçào $\mathcal{C}\left(K^{*}\right)$ sobre o fibrado cotangente $T^{*} Q$ de $Q$, pondo

$$
\mathcal{C}\left(K^{\prime}\right)(q, p)=\frac{1}{k !} p_{\mu_{1}} \ldots p_{\mu_{k}} K^{\mu_{1} \ldots \mu_{k}}(q) .
$$

Observa-se que esta função $\mathcal{C}(K)$ sobre $T^{*} Q$ é um polinômio homogêneo de grau $k$ nos momentos, ou seja, ao longo das fibras de $T^{*} Q$, e que reciprocamente, qualquer funçào desta natureza provém de um campo multivetorial simétrico de ordem $k$ sobre $Q$. A propriedade mais importante desta correspondência é que ela leva o colchete de Schouten entre campos multivetoriais sobre $Q$ para o colchete de Poisson entre funçōes sobre $T^{*} Q$, a menos de um sinal:

$$
\left\{\mathcal{C}\left(K_{1}\right), \mathcal{C}\left(K_{2}\right)\right\}=-\mathcal{C}\left(\left[K_{1}, K_{2}\right]\right) .
$$

Em particular, se $Q$ for uma variedade pseudo-Riemanniana, o (inverso do) tensor métrico g é trivialmente um campo tensorial de Killing de ordem 2, e como

$$
\mathcal{C}(\mathrm{g})=H
$$

é a Hamiltoniana do fluxo geodésico sobre $Q$, concluímos que para um campo multivetorial $K^{\prime}$ de ordem $k$ sobre $Q$ qualquer, a quantidade $\mathcal{C}\left(K^{\circ}\right)$ será uma constante de movimento para o fluxo geodésico sobre $Q$ se e somente se $K$ for um campo tensorial de Kïlling.

O objetivo da presente dissertação é apresentar um tratamento sistemático do fluxo geodésico no espaço-tempo de Kerr, do ponto de vista de teoria dos sistemas Hamiltonianos integráveis. Dentro desta perspectiva, dividimos o trabalho em três capítulos:

No primeiro capítulo sintetizamos os conceitos básicos para uma formulação geométrica da mecânica clássica, válida tanto para a mecânica não-relativística quanto para a mecânica relativística. Como explicamos no início do capítulo, uma diferença crucial entre essas duas versòes da mecânica reside na interpretação do parâmetro das trajetórias, devido à ausência 
do conceito Newtoniano do tempo absoluto e à presença de uma invariância de reparametrização no segundo caso. Na primeira seçào, recapitulamos o formalismo Hamiltoniano em variedades simpléticas, como modelo do espaço de fase do sistema, e na segunda, o tratamento da relação entre simetrias e quantidades conservadas neste contexto, envolvendo conceitos tais como o teorema de Noether, a aplicação momento e a definição de um sistema Hamiltoniano integrável, segundo Liouville. Na terceira seção, apresentamos um breve resumo do formalismo Lagrangiano (princípio da ação estacionária, equações de Euler-Lagrange) e de sua relação com o formalismo Hamiltoniano (transformação de Legendre) para o caso quando o espaço de fase é obtido de um espaço de configuração como o seu fibrado cotangente.

No segundo capítulo discutimos uma classe especial de sistemas Hamiltonianos e Lagrangianos de grande importância e utilidade: o fluxo geodésico em variedades pseudoRiemannianas. Nas duas primeiras seções apresentamos algumas definições básicas e conceitos associados a variedades pseudo-Riemannianas, em particular a noção da derivada covariante de um campo vetorial ou tensorial ao longo de uma curva, que facilita a definição da noção de uma geodésica. Nas duas últimas seções discutimos campos vetoriais e tensoriais de Killing. O primeiro caso é bem conhecido, pois um campo vetorial de Killing $K$ sobre uma variedade pseudo-Riemanniana $Q$ gera um grupo (local) a um parâmetro de isometrias sobre $Q$, que induz um grupo (local) a um parâmetro de simetrias para o fluxo geodésico sobre $T Q$ ou $T^{*} Q$, com quantidades conservadas associadas $\mathcal{C}\left(K^{*}\right)$ que são lineares nas velocidades ou impulsos. O segundo caso, porém, não admite uma interpretação em termos de simetrias que agem sobre a variedade pseudo-Riemanniana original $Q$, pois um campo multivetorial de ordem $>1$ sobre uma variedade $Q$ não gera difeomorfismos de $Q$. No entanto, existe uma interpretação alternativa que se oferece de maneira natural, pois um campo tensorial de Killing $K$ de ordem $k$ sobre uma variedade pseudo-Riemanniana $Q$ sempre gera um grupo (local) a um parâmetro de simetrias para o fluxo geodésico sobre $T Q$ ou $T^{*} Q$, com quantidades conservadas associadas $\mathcal{C}(K)$ que são polinômios homogêneos de grau $k$ nas velocidades ou impulsos: esta ação é simplesmente gerada pelo campo vetorial Hamiltoniano $X_{\mathcal{C}(K)}$ sobre $T Q$ ou $T^{*} Q$ induzido pela função $\mathcal{C}(K)$ sobre $T Q$ ou $T^{*} Q$. Desta forma, a abordagem ao fluxo geodésico do ponto de vista Hamiltoniano oferece uma solução para o antigo problema de dar uma interpretação natural para as simetrias globais que, segundo o teorema de Noether, devem corresponder às quantidades conservadas associadas a tensores de Killing de ordem > 1: basta subir da variedade original ao fibrado tangente ou cotangente para que elas apareçam. Acreditamos que essa interpretação é mais direta e mais simples do que as interpretações dadas por Prince e Caprin $[13,14]$ ) e por Rosquist [16] que, apesar de levarem a resultados equivalentes, utilizam conceitos mais sofisticados introduzidos por Cartan, tais como: geometria diferencial projetiva sobre a variedade produto $\mathbb{R} \times Q$ e sobre o espaço de evolução $\mathbb{R} \times T Q$.

O último capítulo trata do tema central desta dissertação. que é o fluxo geodésico no espaço-tempo de Kerr. Após algumas considerações gerais sobre a interpretação do movimento geodésico em variedades Lorentzianas. como modelo do espaço-tempo na Relatividade Geral, discutimos na primeira seção o papel de grupos de isometrias para reduzir o problema 
de encontrar soluções exatas das equações de Einstein. As soluções exatas conhecidas se dividem em duas grandes classes: as soluções cosmológicas e as soluçòes localizadas, onde as primeiras são soluções com um tensor de energia-momento diferente de zero e geralmente com dependência não-trivial do tempo, descrevendo a distribuição da matéria no universo, enquanto que as segundas são soluçòes no vácuo, i.e., com tensor de energia-momento nulo, e geralmente são estáticas ou pelo menos estacionárias, descrevendo o campo gravitacional gerado por um corpo isolado e em repouso, na região exterior. As soluções mais importantes desta natureza são a solução de Schwarzschild e a solução de Kerr, que descrevemos na segunda e na terceira seção deste capítulo. Em ambos os casos, como aliás em geral, o entendimento do comportamento das geodésicas (tipo tempo e tipo luz) constitui um fator central para o entendimento da física da solução. Neste trabalho, porém, apresentamos apenas aqueles argumentos que se mostram necessários para concluir que o fluxo geodésico no espaço-tempo de Schwarzschild e no espaço-tempo de Kerr constitui um sistema Hamiltoniano integrável, já que existe hoje uma literatura bastante extensa sobre quase todos os demais aspectos do tema: no caso da soluçào de S'chwarzschild, essa discussão faz parte de quase todos os livros texto de Relatividade Geral, tais como [8, 11, 19], enquanto que no caso da solução de Kerr, um tratamento explícito e relativamente elementar do assunto em forma de livro texto é muito mais recente [12].

Finalmente, o trabalho contém três apêndices. No Apêndice A resumimos a definição e as propriedades elementares do colchete de Schouten para campos multivetoriais simétricos. Os Apêndices B e C apresentam demonstrações, baseadas em cálculos longos - mas absolutamente elementares - de um conjunto de fórmulas que dizem respeito à geometria do espaço-tempo de Kerr e que são necessárias para que possamos chegar à conclusão final.

Concluímos esta introdução com algumas observações sobre perspectivas para um trabalho futuro. Um aspecto interessante da métrica de Kerr é que o tensor de Walker-Penrose é do mesmo tipo que o (inverso do) tensor métrico, o que abre a possibilidade de efetuar uma troca entre os dois. Desta forma, e após restrição a um aberto denso adequado, obtém-se uma nova variedade Lorentziana, cujo tensor métrico é o (inverso do) tensor de Walker-Penrose e com um segundo tensor de Killing de ordem 2 que é o (inverso do) tensor métrico original. Este novo tipo de dualidade foi recentemente estudado por vários autores $[7,15]$. No entanto, a nova variedade Lorentziana não é uma solução das equações de Einstein no vácuo, o que prejudica a interpretação do procedimento como uma transformação de dualidade.

Um outro desafio seria encontrar, para o fluxo geodésico no espaço-tempo de Kerr, uma formulação de Lax, o que poderia introduzir técnicas poderosas da teoria de álgebras de Lie. Já existem trabalhos que estendem a formulação de Lax usual de um sistema Hamiltoniano ao caso onde o espaço de fase do sistema é associado a uma variedade pseudo-Riemanniana geral [17], mas o tema ainda parece longe de estar exaurido. 


\section{Capítulo 1}

\section{Mecânica Geométrica}

Neste capítulo tentaremos sintetizar os conceitos básicos para a formulação geométrica da mecânica clássica. Seguindo a convenção que "clássico" significa "não-quântico", a mecânica clássica possui duas versões: a mecânica nào-relativística de Newton e a mecânica relativística de Einstein. A formulação destas duas versões da mecânica é semelhante, havendo inclusive vários objetos matemáticos em comum, apesar de algumas diferenças cruciais de interpretação. Por exemplo, em ambos os casos, a dinâmica de uma partícula sujeita a forças externas e até a dinâmica de sistemas mais complexos, com um número maior de graus de liberdade, é descrita em termos de uma trajetória $\tau \rightarrow q(\tau)$ em uma variedade $Q$ - uma trajetória que é solução de uma equação diferencial ordinária, tipicamente de segunda ordem e tipicamente provinda de uma formulação Lagrangiana (em $T Q$ ) ou Hamiltoniana (em $T^{*} Q$ ). A diferença está na interpretação do parâmetro $\tau$ e da variedade $Q$ :

- Na mecânica não-relativística, a interpretação do formalismo baseia-se tacitamente no axioma de Newton sobre o tempo absoluto:

"Absolute, true, and mathematical time, of itself, and from its own nature, flows equably without relation to anything external, ..."1

Este princípio justifica identificar o parâmetro $\tau$ com este tempo absoluto e assim determina $\tau$ a menos de uma translação $\tau \rightarrow \tau+\tau_{0}$. Esta liberdade remanescente está ligada à possibilidade de relacionar a conservação da energia a uma simetria. A variedade $Q$ é então interpretada como espaço de configuração do sistema: pode ser o espaço no qual a partícula se move, mas pode também ser uma variedade de dimensão $>$ 3, no caso de sistemas sujeitos a vínculos como, por exemplo, o corpo rígido.

\footnotetext{
${ }^{1}$ Sir Isaac Newton - Mathematical Principles of Natural Philosopy and his System of the World: "O tempo absoluto, verdadeiro e matemático. ele próprio, e pela sua própria natureza, flui uniformemente, sem relação com qualquer coisa externa, ..."
} 
- Na mecânica relativística, a interpretação do que é tempo muda radicalmente, pois o tempo absoluto de Newton deixa de existir. O parâmetro $\tau$ é então, pelo menos a priori, apenas um parâmetro, enquanto que a variedade $Q$ é interpretada como o espaço-tempo no qual a partícula se move. Neste caso, é mais difícil incorporar sistemas de partículas com vínculos, pois para definir o que seria um espaço-tempo de configuração seria necessário incluir uma variável tipo tempo para cada partícula envolvida. Por outro lado, temos liberdade completa na escolha do parâmetro $\tau$, i.e., a liberdade de efetuar difeomorfismos $\tau \rightarrow \tau^{\prime}=\tau^{\prime}(\tau)$ quaisquer: diz-se que há invariância por reparametrização. Esta liberdade pode ser restringida pela equação de movimento do sistema, como acontece no caso da equação geodésica, que determina o parâmetro a menos de uma transformação afim $\tau \rightarrow \alpha \tau+\tau_{0}$. No caso de geodésicas tipo tempo, este parâmetro afim é interpretado como o tempo próprio (tempo medido por um relógio transportado ao longo da geodésica) e no caso de geodésicas tipo espaço como a distância espacial (distância entre dois eventos medida ao longo da geodésica), enquanto no caso de geodésicas tipo luz, o parâmetro afim não possui nenhuma interpretação física.

Apesar destas diferenças de interpretação, o formalismo Lagrangiano e o formalismo Hamiltoniano funcionam da mesma forma nas duas versões da mecânica clássica.

Neste trabalho, utilizamos a notação adotada no livro clássico de Abraham-Marsden [1], sendo que a derivada com respeito ao parâmetro $\tau$ de uma trajetória será denotada por um ponto, independentemente da interpretação de $\tau$. Portanto no caso da mecânica relativística. onde as coordenadas locais da variedade $Q$ envolvem uma variável tipo tempo $t$ como, por exemplo, nas soluções de Schwarzschild ou Kerr que se expressam em coordenadas $t, r, v$ e $\varphi$, não deve causar estranheza que apareça uma expressão como $\dot{t}$.

\subsection{Formalismo Hamiltoniano}

Nesta seção introduziremos alguns conceitos matemáticos que são importantes na Mecânica Hamiltoniana, tanto não-relativística quanto relativística, para assim podermos abordar o problema a que nos propomos.

Definição 1.1 Uma variedade simplética é uma dupla $(M, \omega)$, onde $M$ é uma variedade e $\omega$ é uma forma simplética, i.e., uma forma diferencial de grau 2 que é

(i) fechada : $d \omega=0$,

(ii) não-degenerada : para todo ponto $m \in M$ e todo vetor tangente $u_{m} \in T_{m} M$, temos

$$
\omega_{m}\left(u_{m}, v_{m}\right)=0 \text { para todo } v_{m} \in T_{m} M \quad \Longrightarrow \quad u_{m}=0
$$


Definição 1.2 Um sistema Hamiltoniano é uma tripla $(M, \omega, H)$, onde $(M, \omega)$ é uma variedade simplética e $H \in \mathfrak{F}(. M)$.

Definição 1.3 Uma aplicaçào simplética ou, na linguagem utilizada na física, transformação canônica, é uma aplicaçào $\phi: M \rightarrow N$ de uma variedade simplética $M$, com forma simplética $\omega_{M}$, para uma variedade simplética $N$, com forma simplética $\omega_{N}$, tal que

$$
\omega_{M}=\phi^{*} \omega_{N}
$$

Observamos que dada uma variedade simplética, temos para cada $m \in M$ uma forma bilinear antisimétrica sobre o espaço tangente $T_{m} M$ de $M$ em $m$ tal que a aplicação linear

$$
\begin{aligned}
\omega_{m}^{b}: T_{m} M & \longrightarrow T_{m}^{*} M \\
u_{m} & \longmapsto \omega_{m}\left(u_{m}, \cdot\right)
\end{aligned}
$$

induzida por $\omega_{m}$ é um isomorfismo, com inverso denotado por

$$
\begin{aligned}
\omega_{m}^{\sharp}: T_{m}^{*} M & \longrightarrow T_{m} M \\
u_{m}^{*} & \longmapsto\left(\omega_{m}^{b}\right)^{-1}\left(u_{m}^{*}\right)
\end{aligned}
$$

O exemplo padrão de uma variedade simplética é o fibrado cotangente $T^{*} Q$ de uma variedade $Q$ qualquer. De fato, $T^{*} Q$ é uma variedade simplética exata, i.e., munida de uma forma canônica $\theta \in \Omega^{1}\left(T^{*} Q\right)$ tal que $\omega=-d \theta$. Para construir esta forma canônica, consideramos a aplicação tangente $T \tau_{Q}^{*}: T\left(T^{*} Q\right) \rightarrow T Q$ à projeção $\tau_{Q}^{*}: T^{*} Q \rightarrow Q$ e, para $q \in Q, p \in T_{q}^{*} Q, u \in T_{(q, p)}\left(T^{*} Q\right)$, pomos

$$
\theta_{(q, p)}(u)=p\left(T \tau_{Q}^{*}(u)\right) .
$$

Em coordenadas locais $q^{i}$ de $Q$ e usando as coordenadas locais induzidas $\left(q^{i}, \dot{q}^{j}\right)$ de $T Q$, $\left(q^{i}, p_{j}\right)$ de $T^{*} Q$ e $\left(q^{i}, p_{j}, \dot{q}^{k}, \dot{p}_{l}\right)$ de $T\left(T^{*} Q\right)$, temos

$$
\theta=p_{j} d q^{j}
$$

e portanto

$$
\omega=d q^{j} \wedge d p_{j},
$$

onde denotamos por $d q^{i}$ as 1-formas derivadas das funções coordenada $q^{i}$ sobre (abertos de) qualquer uma das variedades $Q, T Q, T^{*} Q$ ou $T\left(T^{*} Q\right)$.

Definição 1.4 Seja $(M, \omega)$ uma variedade simplética. Para toda função $f \in \mathfrak{F}(M)$, chamamos o campo vetorial $X_{f} \in \mathfrak{X}(M)$ sobre $M$ definido por

$$
X_{f}=\omega^{\sharp}(d f),
$$

ou equivalentemente. por

$$
d f=\omega^{b}\left(X_{f}\right)=i_{X, \omega} .
$$

o campo Hamiltoniano associado a $f$. 


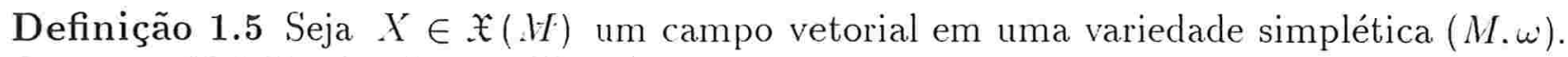
O campo $X$ é dito localmente Hamiltoniano se

$$
\mathfrak{L}_{X} \omega=0
$$

o que significa que o fluxo de $X$ preserva a forma simplética $\omega$.

A última afirmação é uma consequência direta da fórmula [1, p. 91]

$$
\frac{d}{d s}\left(F_{X}\right)_{s}^{*} \omega=\left(F_{X}\right)_{s}^{*}\left(\mathfrak{L}_{X} \omega\right)
$$

que, para uma campo vetorial $X$ qualquer, relaciona a variação da forma simplética $\omega$ ao longo do fluxo $F_{X}$ de $X$ com sua derivada de Lie ao longo de $X$.

A terminologia introduzida na definição anterior pode ser justificada observando-se que

a) todo campo Hamiltoniano é localmente Hamiltoniano,

b) todo campo localmente Hamiltoniano sobre um aberto $U$ de.$I$ que é simplesmente conexo é Hamiltoniano.

De fato, usando a fórmula clássica

$$
\mathfrak{v}_{X}=d i_{X}+i_{X} d
$$

e o fato de que $d \omega=0$, temos

$$
\mathfrak{L}_{X_{f}} \omega=d i_{X_{f}} \omega+i_{X_{f}} d \omega=d i_{X_{f}} \omega=\operatorname{ddf}=0 .
$$

Por outro lado, se $X$ é um campo localmente Hamiltoniano sobre um aberto $U$ de $M$, entào

$$
d\left(i_{X} \omega\right)=\mathfrak{i}_{X} \omega-i_{X} d \omega=-i_{X} d \omega=0 .
$$

Portanto, se $U$ for simplesmente conexo, isto implica que $i_{X} \omega=d f$, ou seja, $X=X_{f}$ para alguma função $f$ sobre $U$.

Definição 1.6 Seja $(M, \omega)$ uma variedade simplética. Para quaisquer duas funções $f, g \in$ $\mathfrak{F}(M)$, chamamos a função $\{f, g\} \in \mathfrak{F}(M)$ definida por

$$
\{f, g\}=\omega\left(X_{f}, X_{g}\right)
$$

onde $X_{f}$ e $X_{g}$ são os campos Hamiltonianos associados a $f$ e $g$, respectivamente, o colchete de Poisson de $f$ e $g$. 
Para uso posterior, notamos mais duas fórmulas envolvendo colchetes de Poisson. Primeiro, para $f, g \in \mathfrak{F}(M)$,

$$
X_{f} \cdot g=\omega^{\sharp}(d f) \cdot g=d g\left(\omega^{\sharp}(d f)\right)=\omega\left(X_{g}, X_{f}\right)=\{g, f\}=-\{f, g\},
$$

ou seja,

$$
X_{f} \cdot g=-\{f, g\}
$$

e segundo, para $f, g, h \in \mathfrak{F}(M)$,

$$
\begin{aligned}
{\left[X_{f}, X_{g}\right] \cdot h } & =X_{f} \cdot\left(X_{g} \cdot h\right)-X_{g} \cdot\left(X_{f} \cdot h\right) \\
& =\{f \cdot\{g, h\}\}-\{g,\{f, h\}\} \\
& =\{\{f \cdot g\}, h\} \\
& =-X_{\{f, g\}} \cdot h,
\end{aligned}
$$

ou seja,

$$
\left[X_{f}, X_{g}\right]=-X_{\{f, g\}}
$$

o que mostra que os campos Hamiltonianos sobre $M$ formam uma subalgebra de Lie $\mathfrak{X}_{H}(M)$ da álgebra de Lie $\mathfrak{X}(M)$ de todos os campos vetoriais sobre $M$ e que a aplicação linear

$$
\begin{aligned}
\mathfrak{F}(M) & \longrightarrow \mathfrak{X}_{H}(M) \\
f & \longmapsto
\end{aligned}
$$

é um anti-homomorfismo de álgebras de Lie, gerando a seguinte sequência exata de álgebras de Lie.

$$
0 \longrightarrow \mathbb{R} \longrightarrow \mathfrak{F}(M) \longrightarrow \mathfrak{X}_{H}(M) \longrightarrow 0,
$$

quando $M$ é conexo. De forma análoga, a fórmula

$$
\mathfrak{L}_{[X, Y]} \omega=\mathfrak{L}_{X} \mathfrak{L}_{Y} \omega-\mathfrak{L}_{Y} \mathfrak{L}_{X} \omega
$$

mostra que os campos localmente Hamiltonianos sobre $M$ também formam uma subalgebra de Lie $\mathfrak{X}_{L H}(M)$ da álgebra de Lie $\mathfrak{X}(M)$ de todos os campos vetoriais sobre $M$.

Qualquer sistema Hamiltoniano é um sistema dinâmico, pois a função $H$ gera um campo vetorial $X_{H} \in \mathfrak{X}(M)$, cujo fluxo descreve a evolução do sistema. As equações diferenciais ordinárias correspondentes são as equações de Hamilton

$$
\dot{x}(\tau)=X_{H}(x(\tau))
$$

onde o ponto, conforme a convenção já mencionada na introdução, denota a derivada com respeito ao parâmetro $\tau$. Para uso posterior, é conveniente, desde já, introduzir o fluxo $F_{X_{H}}$ do campo Hamiltoniano $X_{H}$, que é uma aplicaçào

$$
F_{X_{H}}: \mathcal{D}_{X_{H}} \longrightarrow M
$$


de um aberto $\mathcal{D}_{X_{H}}$ em $\mathbb{R} \times M$ com $\{0\} \times M \subset \mathcal{D}_{X_{H}}$. chamado o domínio do fluxo, para $M$, que em relaçào à primeira variável, que é o parâmetro $\tau$, satisfaz as equações diferenciais ordinárias

$$
\dot{F}_{X_{H}}(\tau, x)=X_{H}\left(F_{X_{H}}(\tau . x)\right)
$$

com condições iniciais

$$
F_{X_{H}}(0, x)=x .
$$

Também notamos que a evolução de uma observável qualquer do sistema, descrita por uma funçào $f \in \mathfrak{F}(M)$, é dada pela equação

$$
\dot{f}=\{f, H\} .
$$

De fato, usando o fluxo Hamiltoniano $F_{X_{H}}$ para estender funções $g$ sobre $M$ a funçòes $g$ sobre $\mathcal{D}_{X_{H}} \subset \mathbb{R} \times M$, pondo $g(\tau, x)=g\left(F_{X_{H}}(\tau, x)\right)$, temos

$$
\begin{aligned}
\dot{f}(\tau, x) & =d f\left(F_{X_{H}}(\tau, x)\right) \cdot \dot{F}_{X_{H}}(\tau, x) \\
& =d f\left(F_{X_{H}}(\tau, x)\right) \cdot X_{H}\left(F_{X_{H}}(\tau, x)\right) \\
& =\omega\left(X_{f}, X_{H}\right)\left(F_{X_{H}}(\tau, x)\right) \\
& =\{f, H\}\left(F_{X_{H}}(\tau, x)\right) \\
& =\{f, H\}(\tau, x) .
\end{aligned}
$$

Exemplo: $M=\mathbb{R}^{2 n}$, com coordenadas lineares $\left(q^{i}, p_{j}\right)(i, j=1, \ldots, n)$ que, consideradas como funções, satisfazem as regras canônicas de comutação

$$
\left\{q^{i}, p_{j}\right\}=\delta_{j}^{i},\left\{q^{i}, q^{j}\right\}=0,\left\{p_{i}, p_{j}\right\}=0 .
$$

Então

$$
X_{H}=\omega^{\sharp}(d H)=\omega^{\sharp}\left(\frac{\partial H}{\partial q^{i}} d q^{i}+\frac{\partial H}{\partial p_{j}} d p_{j}\right),
$$

ou seja,

$$
X_{H}=-\frac{\partial H}{\partial q^{i}} \frac{\partial}{\partial p_{i}}+\frac{\partial H}{\partial p_{i}} \frac{\partial}{\partial q^{i}},
$$

e portanto (1.17) é equivalente a

$$
\begin{aligned}
\dot{q}^{i}(\tau) & =\frac{\partial H}{\partial p_{i}}(q(\tau), p(\tau)), \\
\dot{p}_{j}(\tau) & =-\frac{\partial H}{\partial q^{j}}(q(\tau), p(\tau)) .
\end{aligned}
$$

Além da Hamiltoniana $H$, existem outras funções $f \in \mathfrak{F}(M)$ que são importantes. Por exemplo, na mecânica não-relativística $H$ representa a energia do sistema, enquanto que outras funçòes $f$ representam outras observáveis, tais como momento, momento angular. etc. Portanto. por abuso de linguagem, o campo vetorial $X_{f}$ associado a qualquer funçào $f$ é chamado o campo (vetorial) Hamiltoniano associado a $f$, mesmo quando $f$ nào tem interpretaçào como energia de algum sistema realístico. 
Definição 1.7 Uma quantidade conservada ou integral primeira de um sistema Hamiltoniano é uma função $f \in \mathfrak{F}(. M)$ que é constante ao longo do fluxo Hamiltoniano, i.e., que satisfaz

$$
\{f, H\}=0 \text {. }
$$

A interpretação dual desta relação é que, além de $f$ ser constante ao longo do fluxo de $X_{H}$, $H$ é constante ao longo do fluxo de $X_{f}$, i.e., $X_{f}$ gera um grupo local a um parâmetro de simetrias do sistema Hamiltoniano. Este é o ponto de vista que desenvolveremos na próxima seção.

Exemplo: Obviamente, a própria Hamiltoniana é uma integral primeira, pois

$$
\dot{H}=\{H, H\}=0 .
$$

\subsection{Simetrias e Leis de Conservação: Aplicação Momento}

Vesta seção discutiremos a relação entre a ação de um grupo de Lie sobre o espaço de fase de um sistema Hamiltoniano por simetrias e as quantidades conservadas associadas a esta açào.

Primeiro, lembremos que. dados um grupo de Lie $G$ e uma variedade $M$, uma ação ou operação de $G$ sobre $M$ (por esquerda) é uma aplicação

$$
\begin{aligned}
G \times M & \longrightarrow M \\
(g, m) & \longmapsto g \cdot m
\end{aligned}
$$

com as seguintes propriedades

$$
g_{1} \cdot\left(g_{2} \cdot m\right)=\left(g_{1} g_{2}\right) \cdot m
$$

para $g_{1}, g_{2} \in G$ e $m \in M$ e

$$
1_{G} \cdot m=m
$$

para $m \in M$. Neste caso, para $g \in G$, a aplicação

$$
\begin{aligned}
L_{g}: M & \longrightarrow M \\
m & \longmapsto g \cdot m
\end{aligned}
$$

é um difeomorfismo de $M$, chamado a translação porg (por esquerda), pois $L_{g_{1}} L_{g_{2}}=L_{g_{1} g_{2}}$ e $L_{1_{G}}=i d_{M} ; \operatorname{logo} L_{g}^{-1}=L_{g^{-1}}$. Ademais, cada subgrupo a um parâmetro de $G$, gerado por um elemento $X$ da álgebra de Lie $\mathfrak{g}$, define uma ação

$$
\begin{array}{ccc}
\mathbb{R} \times M & \longrightarrow & M \\
(s \cdot m) & \longmapsto & \exp (s . X) \cdot m
\end{array}
$$


de $\mathbb{R}$ sobre $M$ que representa o fluxo $F_{X_{M}}$ de um campo vetorial $X_{M}$ sobre $M$, chamado campo fundamental sobre $M$ associado a $X$; obviamente

$$
X_{M}(m)=\left.\frac{d}{d s} \exp (s, X) \cdot m\right|_{s=0}
$$

e

$$
F_{X_{M}}(s, m)=\exp (s X) \cdot m
$$

A partir desta definição podemos mostrar que dados dois campos fundamentais $X_{M}$ e $Y_{M}$ associados aos geradores $X^{r}$ e $Y$ em $\mathfrak{g}$, respectivamente, teremos

$$
\left[X_{M}, Y_{M}\right]=-[X, Y]_{M}
$$

o que mostra que a aplicaçào linear

$$
\begin{aligned}
& \mathfrak{g} \longrightarrow \mathfrak{X}(M) \\
& X \longmapsto X_{M}
\end{aligned}
$$

é um anti-homomorfismo de álgebras de Lie.

Definição 1.8 Sejam $G$ um grupo de Lie e $(M, \omega, H)$ um sistema Hamiltoniano. Dizemos que $G$ age sobre este sistema por simetrias se a ação de $G$ sobre $M$ preserva $\omega$ e $H$, i.e., se para todo $g \in G$, vale

$$
L_{g}^{*} \omega=\omega
$$

o que implica.

$$
\left\{f_{1} \circ L_{g}, f_{2} \circ L_{g}\right\}=\left\{f_{1}, f_{2}\right\} \circ L_{g}
$$

para $f_{1}, f_{2} \in \mathfrak{F}(M)$, e

$$
H \circ L_{g}=H \text {. }
$$

Nesta situação, é fácil ver que os campos fundamentais $X_{M}$ sobre $M$ associados aos geradores $X$ em g são localmente Hamiltonianos, o que permite reescrever o anti-homomorfismo (1.35) na seguinte forma:

$$
\begin{aligned}
& \mathfrak{g} \longrightarrow \mathfrak{X}_{L H}(M) \\
& X \longmapsto X_{M}
\end{aligned}
$$

Comparando os dois anti-homomorfismos (1.15) e (1.39), surge a questão se é possível construir um homomorfismo de álgebras de Lie

$$
J: \mathfrak{g} \longrightarrow \mathfrak{F}(M)
$$

tal que (1.39) seja a composição de (1.15) com $J$, i.e., tal que para todo $X$ em $\mathfrak{g}$

$$
\omega^{\sharp}\left(d\left(J\left(X^{\prime}\right)\right)\right)=X_{M},
$$


o que equivale a dizer que para todo $X$ em g e todo campo vetorial $Z$ sobre $M$

$$
Z \cdot J(X)=\omega\left(X_{M}, Z\right) .
$$

É conveniente também considerar a função a valores vetoriais

$$
J: M \longrightarrow \mathrm{g}^{*},
$$

onde $\mathfrak{g}^{*}$ denota o espaço dual de $\mathfrak{g}$, definida pela troca dos argumentos:

$$
J(m)(X) \equiv J(X)(m) .
$$

Definição 1.9 Sejam $(M, \omega, H)$ um sistema Hamiltoniano e $G$ um grupo de Lie que age sobre este sistema por simetrias. Dizemos que o homomorfismo (1.40) ou a função (1.43) é uma aplicação momento associada a esta ação se vale (1.41) ou (1.42).

Observemos que segundo a convenção aqui adotada, uma aplicação momento é necessariamente equivariante no sentido de [1], pois estipulamos que (1.40) seja um homomorfismo de álgebras de Lie, i.e., para $X, Y \in \mathfrak{g}$, temos

$$
\{J(X), J(Y)\}=J([X, Y]) .
$$

Proposição 1.1 Sejam $(M, \omega, H)$ um sistema Hamiltoniano e $G$ um grupo de Lie que age sobre este sistema por simetrias. Então $J$ é uma integral primeira de $X_{H}$ (a valores $\mathrm{em} \mathrm{g}^{*}$ ).

O exemplo mais elementar de uma aplicação momento ocorre quando $\mathfrak{g}$ é a álgebra de Lie abeliana unidimensional $\mathbb{R}$ : neste caso, a aplicação momento equivale a uma quantidade conservada, e podemos afirmar que, no sentido contrário, vale o seguinte

Teorema 1.1 (Noether) Se $f \in \mathfrak{F}(M)$ é uma quantidade conservada e $X_{f} \in \mathfrak{X}(M)$ é completo, então $X_{f}$ gera uma açào do grupo de Lie $\mathbb{R}$ por simetrias.

Para generalizar este teorema ao caso onde g é uma álgebra de Lie abeliana de dimensão qualquer $\mathbb{R}^{l}$, precisamos da seguinte

Definição 1.10 Dizemos que um conjunto $\left\{f_{1}, \ldots, f_{\ell}\right\}$ de quantidades conservadas $f_{j} \in$ $\mathfrak{F}(M)$ está em involução se, além de $\left\{f_{j}, H\right\}=0(j=1, \ldots, \ell)$, temos

$$
\left\{f_{j}, f_{k}\right\}=0 \quad(j, k=1, \ldots, \ell) .
$$

Temos então

Teorema 1.2 (Noether) Se $\left\{f_{1}, \ldots, f_{\ell}\right\}$ é um conjunto de quantidades conservadas $\mathrm{em}$ involução e os $X_{f}, \in \mathfrak{X}(M)$ sào completos. então os $X_{f}$, geram uma açào do grupo de Lie $\mathbb{R}^{i}$ por simetrias. 
$\mathrm{O}$ caso $\ell=\frac{1}{2} \operatorname{dim} M$ é particularmente interessante:

Definição 1.11 (Liouville) Um sistema Hamiltoniano $(M, \omega . H)$ é dito completamente integrável se existe um conjunto $\left\{f_{1}, \ldots, f_{n}\right\}$ de $n$ quantidades conservadas em involução, tal que

1. o número de quantidades conservadas é igual ao número de graus de liberdade do sistema: $n=\frac{1}{2} \operatorname{dim} M$;

2. a Hamiltoniana é uma função das quantidades conservadas, i.e., existe $h \in \mathfrak{F}\left(\mathbb{R}^{n}\right)$ tal que $H=h\left(f_{1}, \ldots, f_{n}\right)$;

3. as quantidades conservadas são funcionalmente independentes, i.e., o conjunto

$$
\sigma(f)=\left\{m \in M / d f_{1}(m), \ldots, d f_{n}(m) \text { sào linearmente dependentes }\right\}
$$

de pontos críticos da aplicação $f=f_{1} \times \cdots \times f_{n}: M \longrightarrow \mathbb{R}^{n}$ tem medida nula.

Existe um teorema geral devido a. Arnold-Liouville que constitui uma generalização do Teorema 1.2, descrevendo a estrutura do espaço de fase de um sistema Hamiltoniano completamente integrável, em particular sua folheação em toros (no caso compacto) [1].

O caso não-abeliano é ainda mais complicado. Contentaremo-nos aqui em apontar uma situação geral em que podemos garantir pelo menos a existência de uma aplicação momento: quando o espaço de fase é uma variedade simplética exata.

Teorema 1.3 Sejam $(M, \omega, H)$ um sistema Hamiltoniano $\in G$ um grupo de Lie que age sobre este sistema por simetrias. Suponhamos ainda que a forma simplética $\omega$ seja exata. i.e., da forma $\omega=-d \theta$ para algum $\theta \in \Omega^{1}(M)$, e que a ação de $G$ preserva a 1-forma $\theta$. Então

$$
J(X)=\theta\left(X_{M}\right)
$$

define uma aplicação momento $J: \mathfrak{g} \longrightarrow \mathfrak{F}(M)$ para esta ação.

Notando que a condição de que a ação de $G$ preserva $\theta$ significa que

$$
L_{g}^{*} \theta=\theta
$$

para $g \in G$ e portanto

$$
\mathfrak{L}_{X_{M}} \theta=0
$$

para $\mathrm{X} \in \mathfrak{g}$, podemos formular uma generalizaçào do teorema ao caso quando a ação de $G$ preserva apenas $\omega$ : 
Teorema 1.4 Sejam (.Y.w.H) um sistema Hamiltoniano $\epsilon$ G um grupo de Lie que age sobre este sistema por simetrias. Suponhamos ainda que a forma simplética $\omega$ seja exata. i.e., da forma $\omega=-d \theta$ para algum $\theta \in \Omega^{1}(M)$, e que existe uma aplicação $f: \mathfrak{g} \longrightarrow \mathfrak{F}(M)$ tal que para todo $X \in \mathfrak{g}$.

$$
\mathfrak{L}_{X_{M}} \theta=d(f(X))
$$

e para todo $\mathrm{X}, Y \in \mathfrak{g}$,

$$
f([X, Y])=Y_{M} \cdot f(X)-X_{M} \cdot f(Y)
$$

Então

$$
J(X)=\theta\left(X_{M}\right)-f(X)
$$

define uma aplicação momento $J: \mathfrak{g} \longrightarrow \mathfrak{F}(M)$ para esta ação.

DemonstraÇÃo. Para mostrarmos que a aplicação definida por (1.50) satisfaz (1.42) e portanto providencia uma aplicação momento, observamos que $\omega=-d \theta$ e, para todo $X \mathrm{em}$ g e todo campo vetorial $Z$ sobre $M$,

$$
d \theta\left(X_{M}, Z\right)=X_{M} \cdot \theta(Z)-Z \cdot \theta\left(X_{M}\right)-\theta\left[X_{M}, Z\right]
$$

ou seja

$$
Z \cdot \theta\left(X_{M}\right)-\omega\left(X_{M}, Z\right)=X_{M} \cdot \theta(Z)-\theta\left(\left[X_{M}, Z\right]\right)=\left(\mathfrak{L}_{X_{M}} \theta\right)(Z),
$$

o que em virtude de (1.48) demonstra (1.42). Em outras palavras, combinando (1.7) e (1.41) (que equivale a (1.42)). provamos que o campo fundamental $X_{M}$ associado ao gerador $X \in \mathfrak{g}$ é exatamente o campo Hamiltoniano associado à função $J\left(X^{\prime}\right)=\theta\left(X_{M}\right)-f(X)$. Usando (1.13) e, em seguida, (1.4\$-1.50), concluímos que

$$
\begin{aligned}
\{J(X), J(Y)\} & =-X_{M} \cdot J(Y)=-X_{M} \cdot \theta\left(Y_{M}\right)+X_{M} \cdot f(Y) \\
& =-\theta\left(\left[X_{M}, Y_{M}\right]\right)-\left(\mathfrak{S}_{X_{M}} \theta\right)\left(Y_{M}\right)+X_{M} \cdot f(Y) \\
& =-\theta\left(\left[X_{M}, Y_{M}\right]\right)-Y_{M} \cdot f(X)+X_{M} \cdot f(Y) \\
& =\theta\left([X, Y]_{M}\right)-f([X, Y]) \\
& =J([X, Y]) .
\end{aligned}
$$

É interessante notar que as duas condições (1.48) e (1.49) são menos restritivas do que parece. Por um lado, o fato de que a ação de $G$ preserva $\omega$ implica que $\mathfrak{L}_{X_{M}} \theta$ deve ser uma 1-forma fechada, e (1.48) afirma que é uma 1-forma exata, o que é automático, por exemplo, quando $M$ for simplesmente conexa. Por outro lado, observando que

$$
\begin{aligned}
& d\left(f([X, Y])-Y_{M} \cdot f(X)+X_{M} \cdot f(Y)\right) \\
& =\mathfrak{L}_{[X, Y]_{M}} \theta+\mathfrak{L}_{X_{M}} d(f(Y))-\mathfrak{L}_{Y_{M}} d(f(X)) \\
& =-\mathfrak{L}_{\left[X_{M}, Y_{M}\right]} \theta+\mathfrak{L}_{X_{M}} \mathfrak{L}_{Y_{M}} \theta-\mathfrak{L}_{Y_{M}} \mathfrak{L}_{X_{M}} \theta \\
& \quad=0,
\end{aligned}
$$


podemos concluir que

$$
f([X, Y])-Y_{M} \cdot f(X)+X_{M} \cdot f(Y)=\omega_{0}(X, Y)
$$

onde $\omega_{0}$ é uma forma bilinear antisimétrica sobre ga a valores reais. Ademais, $\omega_{0}$ é fechada (no sentido de cohomologia de álgebras de Lie), i.e.,

$$
\omega_{0}([X, Y], Z)+\omega_{0}([Y, Z], X)+\omega_{0}([Z, X], Y)=0
$$

pois

$$
\begin{aligned}
\omega_{0}([X, Y], Z)=f([[X . Y], Z]) & +[X, Y]_{M} \cdot f(Z)-Z_{M} \cdot f([X, Y]) \\
=f([[X, Y], Z]) & -X_{M} \cdot\left(Y_{M} \cdot f(Z)\right)-Y_{M} \cdot\left(X_{M} \cdot f(Z)\right) \\
& -Z_{M} \cdot\left(Y_{M} \cdot f(X)\right)+Z_{M} \cdot\left(X_{M} \cdot f(Y)\right),
\end{aligned}
$$

e quando $\omega_{0}$ é exata (no sentido de cohomologia de álgebras de Lie), i.e., quando existe uma forma linear $\theta_{0}$ sobre $\mathfrak{g}$ a valores reais tal que

$$
\omega_{0}(X, Y)=-\theta_{0}([X, Y])
$$

então podemos substituir $f$ por $\tilde{f}$, com

$$
\tilde{f}(X)=f\left(X^{\prime}\right)+\theta_{0}\left(X^{\prime}\right),
$$

para garantir a validade de (1.48) e (1.49), com $f$ substituído por $\tilde{f}$. Portanto, (1.49) também é automática, por exemplo, quando g for semisimples.

\subsection{Formalismo Lagrangiano}

Nas primeiras duas seções, apresentamos o formalismo Hamiltoniano em variedades simpléticas, que na física são interpretadas como o espaço de fase do sistema. Na grande maioria dos sistemas Hamiltonianos encontrados na mecânica este espaço de fase é derivado de um espaço de configuraçào e a Hamiltoniana é derivada de uma Lagrangiana, através de um processo conhecido como a transformação de Legendre. A grande vantagem da formulação Lagrangiana é que ela se relaciona naturalmente com um princípio variacional - o princípio da ação estacionária.

De forma geral, o espaço de configuração de um sistema dinâmico desta natureza é uma variedade que denotaremos por $Q$, com coordenadas locais $q^{i}$, enquanto que o espaço de fase vem em duas versões: o espaço de fase das velocidades que é o fibrado tangente $T Q$ de $Q$, com coordenadas locais $\left(q^{i}, \dot{q}^{j}\right)$, e o espaço de fase dos momentos que é o fibrado cotangente $T^{*} Q$ de $Q$, com coordenadas locais $\left(q^{i}, p_{j}\right)$. A dinâmica da teoria é especificada pela Lagrangiana $L$, que é simplesmente uma funçào $L \in \mathfrak{F}(T Q)$ : esta determina a ação $S$, que é um funcional sobre o "espaço" das curvas entre dois pontos de $Q$ : 
Definição 1.12 Sejam $Q$ uma variedade. $T Q$ seu fibrado tangente e $L \in \mathfrak{F}(T Q)$ uma Lagrangiana. Dado dois pontos $q_{i}, q_{f} \in Q$ e dois pontos $\tau_{i}, \tau_{f} \in \mathbb{R} \operatorname{com} \tau_{i}<\tau_{f}$, e fixando um intervalo aberto $I \subset \mathbb{R}$ com $\tau_{i}, \tau_{f} \in I$, definimos o "espaço" das trajetórias em $Q$ com ponto inicial $\left(\tau_{i}, q_{i}\right)$ e ponto final $\left(\tau_{f}, q_{f}\right)$ por

$$
\Omega\left(\tau_{i}, q_{i} ; \tau_{f}, q_{f}\right)=\left\{\begin{array}{cc}
\gamma: I \rightarrow Q / \begin{array}{c}
\gamma \text { é de classe } C^{2} \text { por partes } \\
\gamma\left(\tau_{i}\right)=q_{i}, \gamma\left(\tau_{f}\right)=q_{f}
\end{array}
\end{array}\right\}
$$

e a ação (relativa ao ponto inicial $\left(\tau_{i}, q_{i}\right)$ e ao ponto final $\left.\left(\tau_{f}, q_{f}\right)\right)$ como sendo a aplicação $S_{\tau_{i}, q_{i} ; \tau_{f}, q_{f}}: \Omega\left(\tau_{i}, q_{i} ; \tau_{f}, q_{f}\right) \rightarrow \mathbb{R}$ definida por

$$
S_{\tau_{i}, q_{i} ; \tau_{f}, q_{f}}(\gamma)=\int_{\tau_{i}}^{\tau_{f}} d \tau L(\gamma(\tau), \dot{\gamma}(\tau))
$$

Normalmente, escrevemos simplesmente $S$ ao invés de $S_{\tau_{i}, q_{i} ; \tau_{f}, q_{f}}$, e para curvas $\gamma_{i}$ em $Q$, escrevemos também $q(\tau)$ ao invés de $\gamma(\tau), \dot{q}(\tau)$ ao invés de $\dot{\gamma}(\tau)$, etc.

Definição 1.13 Sejam $Q$ uma variedade, $T Q$ seu fibrado tangente e $L \in \mathfrak{F}(T Q)$ uma Lagrangiana. Dizemos que uma curva $\hat{i}: I \rightarrow Q$ de classe $C^{2}$ por partes é estacionúria com respeito à ação $S$ induzida pela Lagrangiana $L$ se for um ponto estacionário da ação $S_{T_{i}, q_{i} ; \tau_{f}, q_{f}}$, i.e.,

$$
\delta S(\gamma) \equiv \delta \int_{\tau_{i}}^{\tau_{f}} d \tau L(\gamma(\tau), \dot{\gamma}(\tau))=0
$$

para qualquer escolha de $\tau_{i}, \tau_{f} \in I \operatorname{com} q_{i}=\gamma\left(\tau_{i}\right)$ e $q_{f}=\gamma\left(\tau_{f}\right)$.

Um resultado clássico é a seguinte

Proposição 1.2 Sejam $Q$ uma variedade, $T Q$ seu fibrado tangente $e \quad L \in \mathfrak{F}(T Q)$ uma Lagrangiana. Então uma curva $\gamma: I \rightarrow Q$ de classe $C^{2}$ por partes é estacionária com respeito à ação $S$ induzida pela Lagrangiana $L$ se e somente se satisfaz as equações de Euler-Lagrange

$$
\frac{d}{d \tau} \frac{\partial L}{\partial \dot{q}}-\frac{\partial L}{\partial q}=0,
$$

ou mais explicitamente,

$$
\frac{d}{d \tau} \frac{\partial L}{\partial \dot{q}}(q(\tau), \dot{q}(\tau))-\frac{\partial L}{\partial q}(q(\tau), \dot{q}(\tau))=0
$$

A relação entre o formalismo Lagrangiano e o formalismo Hamiltoniano é fornecida pela transformação de Legendre, definida globalmente como a derivada da Lagrangiana ao longo das fibras de $T Q$ : 
Definição 1.14 Sejam $Q$ uma variedade, $T Q$ seu fibrado tangente e $L \in \mathfrak{F}(T Q)$ uma Lagrangiana. A transformação de Legendre induzida por $L$ é a aplicação $F L: T Q \rightarrow T^{\star} Q$ obtida a partir da derivada de $L$ ao longo das fibras de $T Q$ : definindo, para cada ponto $q \in Q$, a função $L_{q}: T_{q} Q \rightarrow \mathbb{R}$ como sendo a restrição de $L$ a $T_{q} Q$ e a aplicação $(F L)_{q}: T_{q} Q \rightarrow T_{q}^{*} Q$ como sendo a restriçào de $F L$ a $T_{q} Q$, i.e., $L_{q}(\dot{q})=L(q, \dot{q})$ e $(F L)_{q}(\dot{q})=F L(q, \dot{q})$, temos que $(F L)_{q}$ é simplesmente a derivada de $L_{q}$, no sentido usual do cálculo em espaços vetoriais. Em coordenadas locais $q^{i}$ de $Q$ e usando as coordenadas locais induzidas $\left(q^{i}, \dot{q}^{j}\right)$ de $T Q$ e $\left(q^{i}, p_{j}\right)$ de $T^{*} Q$, temos $F L(q, \dot{q})=(q, p)$ com

$$
p_{j}=\frac{\partial L}{\partial \dot{q}^{j}} .
$$

A Lagrangiana $L$ é chamada hiperregular se a transformação de Legendre $F L$ for um difeomorfismo de $T Q$ sobre $T^{*} Q$.

Neste caso, escrevendo $(F L)^{-1}(q, p)=(q, \dot{q})$ e definindo

$$
H(q, p)=p(\dot{q})-L(q, \dot{q})=p_{i} \dot{q}^{i}-L(q, \dot{q}),
$$

as equações de Euler-Lagrange (1.56) associadas a $L$ e as equações de Hamilton (1.24) associadas a $H$ tornam-se equivalentes, sendo que em coordenadas locais $q^{i}$ de $Q$ quaisquer e usando as coordenadas locais induzidas $\left(q^{i}, \dot{q}^{j}\right)$ de $T Q$ e $\left(q^{i}, p_{j}\right)$ de $T^{*} Q$, elas assumem a forma

$$
\frac{d}{d \tau} \frac{\partial L}{\partial \dot{q}^{i}}-\frac{\partial L}{\partial q^{i}}=0
$$

ou mais explicitamente,

$$
\frac{d}{d \tau} \frac{\partial L}{\partial \dot{q}^{i}}(q(\tau), \dot{q}(\tau))-\frac{\partial L}{\partial q^{i}}(q(\tau), \dot{q}(\tau))=0,
$$

$\mathrm{e}$

$$
\begin{gathered}
\dot{q}^{i}=\frac{\partial H}{\partial p_{i}}, \\
\dot{p}_{j}=-\frac{\partial H}{\partial q^{j}},
\end{gathered}
$$

ou mais explicitamente,

$$
\begin{aligned}
\dot{q}^{i}(\tau) & =\frac{\partial H}{\partial p_{i}}(q(\tau), p(\tau)), \\
\dot{p}_{j}(\tau) & =-\frac{\partial H}{\partial q^{j}}(q(\tau), p(\tau)) .
\end{aligned}
$$

Finalmente, o tratamento de simetrias em sistemas Hamiltonianos sobre um espaço de fase derivado de um espaço de configuração e com Hamiltoniana derivada de uma Lagrangiana. através da transformaçào de Legendre, se encaixa nas hipóteses do Teorema 1.3: 
Teorema 1.5 Sejam $Q$ uma variedade, $T Q$ seu fibrado tangente, $M=T^{*} Q$ seu fibrado cotangente, $L \in \mathfrak{F}(T Q)$ uma Lagrangiana hiperregular $e H \in \mathfrak{F}\left(T^{*} Q\right)$ a Hamiltoniana correspondente. Seja $G$ um grupo de Lie que age sobre $Q$ tal que a ação induzida de $G$ sobre $T Q$ preserva a Lagrangiana $L$, o que é equivalente a dizer que a ação induzida de $G$ sobre $T^{*} Q$ preserva a Hamiltoniana $H$. Então

$$
J(X)(q, p)=p\left(X_{Q}(q)\right)=p_{i} X_{Q}^{i}(q)
$$

define uma aplicação momento $J: \mathfrak{g} \longrightarrow \mathfrak{F}(M)$ para esta ação.

De fato, como a ação de $G$ sobre $T^{*} Q$ é induzida por uma ação de $G$ sobre $Q$, a preservação da 1 -forma canônica $\theta$ sobre $T^{*} Q$, dada por (1.4), é automática. 


\section{Capítulo 2}

\section{Fluxo Geodésico}

A meta deste capítulo é discutir o fluxo geodésico em variedades pseudo-Riemannianas. que constitui um exemplo importante de um sistema Lagrangiano e Hamiltoniano. Por um lado, na mecânica não-relativística, geodésicas são trajetórias de partículas livres, tanto no espaço Euclideano (movimento retilíneo uniforme) como em variedades curvas. Um exemplo explícito é o fluxo geodésico no grupo de Lie $S U(2)$, equipado com uma métrica invariante por direita, que descreve o corpo rígido [1, pp. 311-337]: este é um exemplo importante de um sistema completamente integrável, com quantidades conservadas em involução que são a energia $H$, o quadrado do momento angular total $L^{2}$ e um componente do momento angular. digamos $L_{3}$. Mais geralmente. Mishchenko e Fomenko mostraram que o fluxo geodésico em um grupo de Lie compacto, pelo menos quando equipado com uma métrica bi-invariante, é sempre completamente integrável [6].

Por outro lado, na mecânica relativística, geodésicas (tipo tempo ou tipo luz) são as trajetórias de partículas em queda livre, porém com algumas mudanças substanciais de interpretação: a variedade ambiente agora é o espaço-tempo, ao invés do espaço, e as trajetórias de partículas correspondem às linhas de universo de Einstein, sendo que para partículas com massa de repouso diferente de zero estas são curvas tipo tempo, enquanto que para partículas com massa de repouso igual a zero (fótons e talvez neutrinos), são curvas tipo luz. Ademais, o campo gravitacional é geometrizado, e portanto a expressão "em queda livre" caracteriza o movimento na ausência de forças externas, exceto forças gravitacionais. No entanto, o conceito de um sistema completamente integrável continua sendo de considerável importância e utilidade.

\subsection{Geometria pseudo-Riemanniana}

Definição 2.1 Uma variedade pseudo-Riemanniana é uma clupla ( $M . g$ ), onde $M$ é uma variedade e g é um campo tensorial simétrico e covariante de grau 2, i.e., g $\in \Gamma\left(\bigvee^{2} T^{*} M\right)$, 
chamado o tensor métrico ou simplesmente a métrica, que é nào-degenerado : para todo ponto $m \in M$ e todo vetor tangente $u_{m} \in T_{m} M$, temos

$$
\mathrm{g}\left(u_{m}, v_{m}\right)=0 \text { para todo } v_{m} \in T_{m} M \Longrightarrow u_{m}=0 .
$$

Métricas positivas definidas são chamadas de métricas Riemannianas e métricas com índice 1 ou $n-1$ são chamadas de métricas Lorentzianas. A respeito desta última definição, há duas convenções possíveis, e ambas são usadas na literatura. Neste trabalho, adotaremos a convenção da área de Relatividade Geral, que é a primeira, significando que o tensor métrico, em cada ponto da variedade e em uma base ortonormal do espaço tangente neste ponto, assume a forma $\operatorname{diag}(-1,1, \ldots 1)$.

O exemplo mais simples de uma variedade de Lorentz é o espaço-tempo de Minkowski, que já é um conceito central da Relatividade Restrita. Matematicamente é a variedade $\mathbb{R}^{4}$ com a métrica Lorentziana plana, que em coordenadas cartesianas $\left(x^{0}, x^{1}, x^{2}, x^{3}\right)$ pode ser expressa na forma

$$
d s^{2}=-\left(d x^{0}\right)^{2}+\left(d x^{1}\right)^{2}+\left(d x^{2}\right)^{2}+\left(d x^{4}\right)^{2} .
$$

A arena da teoria da Relatividade Geral são as variedades de Lorentz e várias noções importantes derivam simplesmente da geometria dos espaços tangentes de tais variedades, para onde é transferida toda geometria do espaço de Minkowski.

Definição 2.2 Seja $(M, \mathrm{~g})$ uma variedade de Lorentz. Para qualquer ponto $m$ de $M$, dizemos que um vetor tangente $v \in T_{m} M$ é

- tipo espaço se $\mathrm{g}_{m}(v, v)>0$;

- tipo tempo se $\mathrm{g}_{m}(v, v)<0$;

- tipo luz se $g_{m}(v, v)=0$ e $v \neq 0$.

Os vetores tipo tempo e os vetores tipo luz definem um cone $\mathcal{T}$ e um cone $\Lambda$, chamados o cone do tempo e o cone de luz, respectivamente. Cada um destes é a união disjunta de dois cones convexos, o cone do passado $\mathcal{T}^{-}$ou $\Lambda^{-}$e o cone do futuro $\mathcal{T}^{+}$ou $\Lambda^{+}$, onde

$$
\begin{aligned}
& \mathcal{T}^{+}=\left\{v \in \mathcal{T} / v^{0}>0\right\}, \\
& \mathcal{T}^{-}=\left\{v \in \mathcal{T} / v^{0}<0\right\}, \\
& \Lambda^{+}=\left\{v \in \Lambda / v^{0}>0\right\}, \\
& \Lambda^{-}=\left\{v \in \Lambda / v^{0}<0\right\},
\end{aligned}
$$

sendo que $\Lambda^{+}$e $\Lambda^{-}$sào difeomorfos a $\mathbb{R}^{+} \times S^{n-2}$, e temos ainda que

$$
\partial \mathcal{T}^{ \pm}=\Lambda^{ \pm} \cup\{0\}
$$




\subsection{Fluxo Geodésico}

Nesta seção introduziremos inicialmente alguns conceitos associados à noção de derivada covariante de campos vetoriais e tensoriais ao longo de curvas. Estas definições conduzirão ao conceito de geodésica.

Seja $\gamma: I \rightarrow Q$ uma curva em $Q$; escrevemos também $q(\tau)$ ao invés de $\gamma(\tau)$. Definimos a derivada covariante de um campo vetorial $\mathrm{X}$ ao longo da curva $\gamma$ como sendo o campo vetorial

$$
\frac{D . X}{d \tau}
$$

ao longo da curva $\gamma$ definido por

$$
\frac{D X^{\mu}}{d \tau}(\tau)=\frac{d X^{\mu}}{d \tau}(\tau)+\Gamma_{\kappa \lambda}^{\mu}(q(\tau)) \dot{q}^{\kappa}(\tau) X^{\lambda}(\tau),
$$

onde os símbolos de Christoffel $\Gamma_{n \lambda}^{\mu}$ são dados por

$$
\Gamma_{\kappa \lambda}^{\mu}=\frac{1}{2} \mathrm{~g}^{\mu \nu}\left(\partial_{\kappa} \mathrm{g}_{. \nu}+\partial_{\lambda} \mathrm{g}_{\kappa \nu}-\partial_{\nu} \mathrm{g}_{\kappa, \lambda}\right)
$$

Este procedimento pode ser generalizado para definir a derivada covariante de um campo tensorial $S$ qualquer ao longo da curva $\gamma$,

$$
\begin{aligned}
\frac{D S_{\nu_{1} \ldots \nu_{q}}^{\mu_{1} \ldots \mu_{p}}}{d \tau}(\tau)= & \frac{d S_{\nu_{1} \ldots \nu_{q}}^{\mu_{1} \ldots \mu_{p}}}{d \tau}(\tau)+\sum_{i=1}^{p} \Gamma_{\kappa, \lambda}^{\mu_{i}}(q(\tau)) \dot{q}^{\kappa}(\tau) S_{\nu_{1} \ldots \nu_{q}}^{\mu_{1} \ldots \mu_{i-1} \lambda \mu_{i+1} \ldots \mu_{p}}(\tau) \\
& -\sum_{j=1}^{q} \Gamma_{\kappa \nu_{j}}^{\lambda}(q(\tau)) \dot{q}^{\kappa}(\tau) S_{\nu_{1} \ldots \nu_{j-1} \lambda \nu_{j+1} \ldots \nu_{q}}^{\mu_{1} \ldots \mu_{p}}(\tau)
\end{aligned}
$$

No caso onde $X$ ou $S$ provêm de um campo vetorial $\tilde{X}$ ou de um campo tensorial $\tilde{S}$ sobre $Q$ por composição com a curva, i.e., $X(\tau)=\tilde{X}^{\prime}(q(\tau))$ ou $S(\tau)=\tilde{S}(q(\tau))$, a derivada covariante ao longo da curva é simplesmente a derivada covariante usual na direção tangencial:

$$
\begin{gathered}
\frac{D X^{\mu}}{d \tau}(\tau)=\dot{q}^{\kappa}(\tau)\left(\nabla_{\kappa} \tilde{X}^{\mu}\right)(q(\tau)), \\
\frac{D S_{\nu_{1} \ldots \nu_{q}}^{\mu_{1} \ldots \mu_{p}}}{d \tau}(\tau)=\dot{q}^{\kappa}(\tau)\left(\nabla_{\kappa} \tilde{S}_{\nu_{1} \ldots \nu_{q}}^{\mu_{1} \ldots \mu_{p}}\right)(q(\tau)),
\end{gathered}
$$

No que segue, omitiremos o símbolo . .

A derivada covariante comuta com contraçòes e com o produto tensorial, no sentido de satisfazer uma regra de Leibniz com respeito a cada um. (Para funções, a derivada covariante coincide com a derivada comum, por definiçào.) 
Definição 2.3 Dizemos que o campo $X$ ou $S$ é paralelo ao longo da curva $\gamma$ se

$$
\frac{D X}{d \tau}=0 \text { ou } \frac{D S}{d \tau}=0
$$

e dizemos que uma curva $\gamma$ é uma geodésica se ela é autoparalela, i.e., se a derivada $\dot{\gamma}$ de $\hat{i}$ é paralela ao longo de $\gamma$,

$$
\frac{D}{d \tau} \frac{d q}{d \tau}=0
$$

ou seja, se vale a єquação geodésica

$$
\ddot{q}^{\mu}+\Gamma_{\kappa \lambda}^{\mu}(q) \dot{q}^{\kappa} \dot{q}^{\lambda}=0 .
$$

Exemplo: No caso da métrica de Minkowski, temos

$$
\Gamma_{r \lambda}^{\mu}=0,
$$

e portanto a equaçào geodésica se reduz a

$$
\ddot{q}^{\mu}=0,
$$

ou seja, as geodésicas no espaço-tempo de Minkowski são linhas retas.

Queremos mostrar agora que para uma variedade pseudo-Riemanniana $(Q, \mathrm{~g})$ qualquer. a equação geodésica é a equação de Euler-Lagrange de um sistema dinâmico, com a própria variedade $Q$ como espaço de configuração, que admite uma formulação Lagrangiana no fibrado tangente $T Q$ (espaço de fase das velocidades) e uma formulação Hamiltoniana no fibrado cotangente $T^{*} Q$ (espaço de fase dos momentos). Este sistema dinâmico é chamado o fluro geodésico em $Q$. De fato, a Lagrangiana $L$ e a Hamiltoniana $H$ são as mais simples possiveis:

$$
\begin{aligned}
& L(q, \dot{q})=\frac{1}{2} \mathrm{~g}_{\mu \nu}(q) \dot{q}^{\mu} \dot{q}^{\nu}, \\
& H(q, p)=\frac{1}{2} \mathrm{~g}^{\mu \nu}(q) p_{\mu} p_{\nu},
\end{aligned}
$$

sendo relacionadas pela transformação de Legendre dada por

$$
p_{\mu}=\frac{\partial L}{\partial \dot{q}^{\mu}}=g_{\mu \nu}(q) \dot{q}^{\nu} .
$$

Esta Lagrangiana é hiperregular, pois a transformação de Legendre é inversível:

$$
\dot{q}^{\mu}=\frac{\partial H}{\partial p_{\mu}}=\mathrm{g}^{\mu \nu}(q) p_{\nu} .
$$

Teorema 2.1 Seja $(Q, \mathrm{~g})$ uma variedade pseudo-Riemanniana. Então as equaçôes de EulerLagrange (1.55) associadas à Lagrangiana (2.10) são as equações geodésicas (2.9). 
Demonstração. Para a Lagrangiana $L$ dada por (2.10), temos

$$
\begin{aligned}
\frac{d}{d \tau} \frac{\partial L}{\partial \dot{q}^{\mu}}-\frac{\partial L}{\partial q^{\mu}} & =\frac{d}{d \tau}\left(\mathrm{g}_{\mu \nu}(q) \dot{q}^{\nu}\right)-\frac{1}{2}\left(\partial_{\mu} \mathrm{g}_{\kappa \lambda}\right)(q) \dot{q}^{\kappa} \dot{q}^{\lambda} \\
& =\mathrm{g}_{\mu \nu}(q) \ddot{q}^{\nu}+\left(\partial_{\sigma} \mathrm{g}_{\mu \nu}\right)(q) \dot{q}^{\nu} \dot{q}^{\sigma}-\frac{1}{2}\left(\partial_{\mu} \mathrm{g}_{\kappa \lambda}\right)(q) \dot{q}^{\kappa} \dot{q}^{\lambda} \\
& =\mathrm{g}_{\mu \nu}(q) \ddot{q}^{\nu}+\frac{1}{2}\left(\partial_{\kappa} \mathrm{g}_{\lambda \mu}+\partial_{\lambda} \mathrm{g}_{\kappa \mu}-\partial_{\mu} \mathrm{g}_{\kappa \lambda}\right)(q) \dot{q}^{\kappa} \dot{q}^{\lambda} \\
& =\mathrm{g}_{\mu \nu}(q)\left(\ddot{q}^{\nu}+\Gamma_{\kappa \lambda}^{\nu}(q) \dot{q}^{\kappa} \dot{q}^{\lambda}\right)
\end{aligned}
$$

Os cálculos envolvendo o fluxo geodésico são significativamente simplificados quando substituímos todas as derivadas comuns ao longo de curvas por derivadas covariantes. Neste sentido, e seguindo o princípio usado na construção das fórmulas (2.2) e (2.4), definimos

$$
\begin{gathered}
\frac{D q^{\mu}}{d \tau}(\tau) \equiv \frac{d q^{\mu}}{d \tau}(\tau)=\dot{q}^{\mu}(\tau), \\
\frac{D \dot{q}^{\mu}}{d \tau}(\tau)=\frac{d \dot{q}^{\mu}}{d \tau}(\tau)+\Gamma_{\kappa \lambda}^{\mu}(q(\tau)) \dot{q}^{\kappa}(\tau) \dot{q}^{\top}(\tau) . \\
\frac{D p_{\mu}}{d \tau}(\tau)=\frac{d p_{\mu}}{d \tau}(\tau)-\Gamma_{\kappa \mu}^{\nu}(q(\tau)) \dot{q}^{\kappa}(\tau) p_{\nu}(\tau) .
\end{gathered}
$$

Evidencia-se assim a equivalência entre (2.8) e (2.9). Ademais. a forma Hamiltoniana das equações geodésicas torna-se extremamente simples:

$$
\begin{gathered}
\frac{D q^{\mu}}{d \tau}=\mathrm{g}^{\mu \nu}(q) p_{\nu}, \\
\frac{D p_{\mu}}{d \tau}=0 .
\end{gathered}
$$

\subsection{Isometrias e Campos Vetoriais de Killing}

É bem conhecido que em variedades pseudo-Riemannianas os campos vetoriais que geram subgrupos (locais) a um parâmetro de isometrias são os campos vetoriais de Killing. Por outro lado, os campos vetoriais de Killing também geram simetrias para o fluxo geodésico, com quantidades conservadas que são lineares nas velocidades ou impulsos. Como veremos na próxima seção, este último ponto de vista admite uma generalização que é crucial para o entendimento da integrabilidade do fluxo geodésico na solução de Kerr.

Definição 2.4 Uma isometria é uma aplicação $\phi: M \rightarrow V$ de uma variedade pseudoRiemanniana $M$, com tensor métrico $\mathrm{g}_{M}$, para uma variedade pseudo-Riemanniana $N$, com tensor métrico $g_{N}$, tal que

$$
g_{M}=\phi^{*} g_{N} .
$$


Definição 2.5 Seja $X \in \mathfrak{X}(Q)$ um campo vetorial em uma variedade pseudo-Riemanniana $(Q, \mathrm{~g})$. O campo $X$ é dito campo vetorial de Killing se

$$
\mathfrak{\sim}_{X} \mathrm{~g}=0
$$

o que significa que o fluxo de $X$ preserva o tensor métrico g.

A última afirmação é uma consequência direta da fórmula [1, p. 91]

$$
\frac{d}{d s}\left(F_{X}\right)_{s}^{*} \mathrm{~g}=\left(F_{X}\right)_{s}^{*}\left(\mathfrak{L}_{X} \mathrm{~g}\right)
$$

que, para um campo vetorial $X$ qualquer, relaciona a variação do tensor métrico $g$ ao longo do fluxo $F_{X}$ de $X$ com sua derivada de Lie ao longo de $X$.

A condição na definição anterior pode ser reformulada em termos de derivadas covariantes, com respeito à conexão de Levi-Civita $\nabla$ :

Proposição 2.1 Seja $X \in \mathfrak{X}(Q)$ um campo vetorial em uma variedade pseudo-Riemanniana $(Q, \mathrm{~g})$. Então $X$ é um campo vetorial de Killing se e somente se satisfaz a equação de Killing

$$
\nabla_{\mu} X_{\nu}+\nabla_{\nu} X_{\mu}=0
$$

DemonstraÇÃo. A equivalência de (2.18) e 2.20) segue do seguinte cálculo:

$$
\begin{aligned}
\left(\mathfrak{L}_{X} \mathrm{~g}\right)\left(\partial_{\mu}, \partial_{\nu}\right) & =X \cdot \mathrm{g}_{\mu \nu}-\mathrm{g}\left(\mathfrak{L}_{X} \partial_{\mu}, \partial_{\nu}\right)-\mathrm{g}\left(\partial_{\mu}, \mathfrak{L}_{X} \partial_{\nu}\right) \\
& =\mathrm{g}\left(\nabla_{X} \partial_{\mu}, \partial_{\nu}\right)+\mathrm{g}\left(\partial_{\mu}, \nabla_{X} \partial_{\nu}\right)-\mathrm{g}\left(\mathfrak{L}_{X} \partial_{\mu}, \partial_{\nu}\right)-\mathrm{g}\left(\partial_{\mu}, \mathfrak{L}_{X} \partial_{\nu}\right) \\
& =\mathrm{g}\left(\nabla_{\mu} X, \partial_{\nu}\right)+\mathrm{g}\left(\partial_{\mu}, \nabla_{\nu} X\right) \\
& =\nabla_{\mu} X_{\nu}+\nabla_{\nu} X_{\mu} .
\end{aligned}
$$

\section{Exemplo:}

(a) No espaço Euclideano $\mathbb{R}^{n}$ temos os campos vetoriais de Killing

$$
P_{i}=\frac{\partial}{\partial q^{i}} \quad, \quad L_{i j}=q^{i} \frac{\partial}{\partial q^{j}}-q^{j} \frac{\partial}{\partial q^{i}}
$$

$(i, j=1, \ldots, n)$, gerando translações e rotações, respectivamente.

(b) No espaço de Minkowski $\mathbb{R}^{n}$ temos os campos vetoriais de Killing

$$
\begin{gathered}
P_{0}=\frac{\partial}{\partial t} \quad, \quad P_{i}=\frac{\partial}{\partial q^{i}} \\
B_{i}=q^{i} \frac{\partial}{\partial t}-t \frac{\partial}{\partial q^{i}} \quad, \quad L_{i j}=q^{i} \frac{\partial}{\partial q^{j}}-q^{j} \frac{\partial}{\partial q^{i}}
\end{gathered}
$$

$(i, j=1, \ldots, n-1)$, gerando translaçòes (temporais e espaciais), "boosts" e rotaçòes. respectivamente. 
Suponha agora que $Q$ seja uma variedade pseudo-Riemanniana e $G$ um grupo de Lie que age sobre $Q$ por isometrias, i.e., para todo $g \in G$, a translação por esquerda $L_{g}: Q \rightarrow Q$ é uma isometria de $Q$. Neste caso, é óbvio que a Lagrangiana (2.10) do fluxo geodésico será invariante sob a ação induzida de $G$ sobre o fibrado tangente $T Q$ de $Q$ e a Hamiltoniana (2.11) do fluxo geodésico será invariante sob a ação induzida de $G$ sobre o fibrado cotangente $T^{*} Q$ de $Q$. Portanto, segundo o Teorema 1.5, a fórmula (1.65) providencia uma aplicaçào momento para esta situação.

Tendo em vista a generalização a ser introduzida na próxima seção, efetuaremos aqui uma ligeira mudança de notação: dado um campo vetorial de Killing $Z$ sobre $Q$, chamamos a quantidade conservada ou carga que lhe corresponde de $\mathcal{C}(Z)$, sendo que a relação com a aplicação momento definida pela fórmula (1.65) é que para um gerador $X \in \mathfrak{g}$ com campo vetorial de Killing $X_{Q} \in \mathfrak{X}(Q)$ associada, $J(X)=\mathcal{C}\left(X_{Q}\right)$. Explicitamente, introduzimos a seguinte aplicaçào linear

$$
\begin{aligned}
\mathcal{C}: \mathfrak{X}(Q) & \longrightarrow \mathfrak{F}\left(T^{*} Q\right) \\
Z & \longmapsto \mathcal{C}(Z)
\end{aligned}
$$

onde

$$
\mathcal{C}(Z)(q, p)=p_{\mu} Z^{\mu}(q)
$$

Notamos que a aplicação (2.21) é um anti-homomorfismo de álgebras de Lie, i.e.,

$$
\left\{\mathcal{C}\left(Z_{1}\right), \mathcal{C}\left(Z_{2}\right)\right\}=-\mathcal{C}\left(\left[Z_{1}, Z_{2}\right]\right)
$$

(veja $(1.45)$ e (1.34)) e tal que $\mathcal{C}(Z)$ é uma quantidade conservada sob o fluxo geodésico em $Q$, i.e.,

$$
\{\mathcal{C}(Z), H\}=0
$$

com $H$ dada por (2.11), se e somente se $Z$ for um campo vetorial de Killing. De fato, temos

$$
\begin{aligned}
\left\{\mathcal{C}\left(Z_{1}\right), \mathcal{C}\left(Z_{2}\right)\right\} & =\left\{p_{\mu} Z_{1}^{\mu}, p_{\nu} Z_{2}^{\nu}\right\}=p_{\mu} Z_{2}^{\nu} \partial_{\nu} Z_{1}^{\mu}-Z_{1}^{\mu} p_{\nu} \partial_{\mu} Z_{2}^{\nu} \\
& =-p_{\mu}\left[Z_{1}, Z_{2}\right]^{\mu}=-\mathcal{C}\left(\left[Z_{1}, Z_{2}\right]\right)
\end{aligned}
$$

e usando (2.16). (2.12), (2.5), o fato de que o tensor métrico é covariantemente constante e, finalmente, $(2.20)$, concluímos que

$$
\begin{aligned}
\frac{d}{d \tau}(\mathcal{C}(Z)) & =\frac{d}{d \tau}\left(p_{\mu} Z^{\mu}\right)=\frac{D p_{\mu}}{d \tau} Z^{\mu}+p_{\mu} \frac{D Z^{\mu}}{d \tau} \\
& =g_{\mu \kappa} \dot{q}^{\kappa} \dot{q}^{\curlywedge} \nabla_{\lambda} Z^{\mu}=\dot{q}^{\kappa} \dot{q}^{\curlywedge} \nabla_{\lambda} Z_{\kappa}=0 .
\end{aligned}
$$

A característica desta construção é que ela fornece apenas cargas conservadas que são lineares nos momentos. Na próxima seção, veremos como generalizar o procedimento para produzir cargas conservadas que são polinomiais nos momentos; isto leva naturalmente ao conceito de campos tensoriais de Killing. 


\subsection{Campos Tensoriais de Killing}

Começamos com uma definiçào:

Definição 2.6 Seja $K \in \mathfrak{X}^{k}(Q)$ um campo multivetorial simétrico de grau $k$ em uma variedade pseudo-Riemanniana $(Q, \mathrm{~g})$. O campo $K$ é dito campo tensorial de Killing se

$$
[K, \mathrm{~g}]=0,
$$

onde [., .] denota o colchete de Schouten (veja Apêndice A).

Novamente, a condição nesta definição pode ser reformulada em termos de derivadas covariantes com respeito à conexão de Levi-Civita $\nabla$ :

Proposição 2.2 Seja $i \in \in \mathfrak{X}^{k}(Q)$ um campo multivetorial simétrico de grau $k$ em uma variedade pseudo-Ritmanniana $(Q, \mathrm{~g})$. Então $I \dot{e}$ é um campo tensorial de Killing se $\epsilon$ somente se satisfaz a equaçào de Killing

$$
\nabla_{(\nu} K_{\left.\mu_{1} \ldots \mu_{k}\right)}=0,
$$

onde os parênteses indicam simetrização total.

Demonstração. Segundo (A.15) e usando o fato de que o tensor métrico é covariantemente constante, temos

$$
\left[K^{\prime}, \mathrm{g}\right]^{\mu_{1} \ldots \mu_{k} \nu}=-(k+1) \mathrm{g}^{\lambda(\nu} \nabla_{\lambda} \Lambda^{\left.\cdot \mu_{1} \ldots \mu_{k}\right)}
$$

e portanto

$$
\left[K^{\prime}, g\right]_{\mu_{1} \ldots \mu_{k} \nu}=-(k+1) \nabla_{(\nu} K_{\left.\mu_{1} \ldots \mu_{k}\right)}
$$

o que torna a equivalência de (2.25) e (2.26) óbvia.

Isso posto, podemos introduzir a seguinte aplicaçào linear:

$$
\begin{aligned}
\mathcal{C}: \mathfrak{X}^{k}(Q) & \longrightarrow \mathfrak{F}\left(T^{*} Q\right) \\
K^{k} & \longmapsto \mathcal{C}\left(K^{*}\right)
\end{aligned}
$$

onde

$$
\mathcal{C}\left(K^{*}\right)(q, p)=\frac{1}{k !} p_{\mu_{1}} \ldots p_{\mu_{k}} K^{\mu_{1} \ldots \mu_{k}}(q) .
$$

Novamente, a aplicação (2.29) é um anti-homomorfismo de álgebras de Lie, i.e.,

$$
\left\{\mathcal{C}\left(K_{1}\right), \mathcal{C}\left(K_{2}\right)\right\}=-\mathcal{C}\left(\left[K_{1}, K_{2}\right]\right),
$$

e tal que $\mathcal{C}\left(K^{\circ}\right)$ é uma quantidade conservada sob o fluxo geodésico em $Q$, i.e.,

$$
\left\{\mathcal{C}\left(K^{\circ}\right), H\right\}=0 .
$$


com $H$ dada por (2.11). se e somente se $K$ for um campo tensorial de Kiilling. De fato. temos

$$
\begin{aligned}
& \left\{\mathcal{C}\left(K_{1}\right), \mathcal{C}\left(K_{2}\right)\right\}=\frac{1}{k_{1} !} \frac{1}{k_{2} !}\left\{p_{\mu_{1}} \ldots p_{\mu_{k_{1}}} K_{1}^{\mu_{1} \ldots \mu_{k_{1}}}, p_{\nu_{1}} \ldots p_{\nu_{k_{2}}} K_{2}^{-\nu_{1} \ldots \nu_{k_{2}}}\right\} \\
& =\frac{1}{k_{1} !} \frac{1}{k_{2} !}\left(\frac{\partial}{\partial q^{\lambda}}\left(p_{\mu_{1}} \ldots p_{\mu_{k_{1}}} K_{1}^{{ }^{\mu} \ldots \mu_{1} \ldots \mu_{k_{1}}}\right) \frac{\partial}{\partial p_{\lambda}}\left(p_{\nu_{1}} \ldots p_{\nu_{k_{2}}} K_{2}^{\prime \nu_{1} \ldots \nu_{k_{2}}}\right)\right. \\
& \left.-\frac{\partial}{\partial p_{\lambda}}\left(p_{\mu_{1}} \ldots p_{\mu_{k_{1}}} K_{1}^{\mu_{1} \ldots \mu_{k_{1}}}\right) \frac{\partial}{\partial q^{\lambda}}\left(p_{\nu_{1}} \ldots p_{\nu_{k_{2}}} K_{2}^{\nu_{1} \ldots \nu_{k_{2}}}\right)\right) \\
& =\frac{1}{k_{1} !} \frac{1}{k_{2} !}\left(k_{2} p_{\mu_{1}} \ldots p_{\mu_{k_{1}}} p_{\nu_{1}} \ldots p_{\nu_{k_{2}-1}} \partial_{\lambda} K_{1}^{-\mu_{1} \ldots \mu_{k_{1}}} K_{2}^{\lambda \nu_{1} \ldots \nu_{k_{2}-1}}\right. \\
& \left.-k_{1} p_{\mu_{1}} \ldots p_{\mu_{k_{1}-1}} p_{\nu_{1}} \ldots p_{\nu_{k_{2}}} K_{1}^{\lambda \mu_{1} \ldots \mu_{k_{1}-1}} \partial_{\lambda} K_{2}^{-\nu_{1} \ldots \nu_{k_{2}}}\right) \\
& =-p_{\sigma_{1}} \ldots p_{\sigma_{k_{1}+k_{2}-1}}\left(\frac{1}{\left(k_{1}-1\right) ! k_{2} !} K_{1}^{-\lambda \sigma_{1} \ldots \sigma_{k_{1}-1}} \partial_{\lambda} K_{2}^{. \sigma_{k_{1}} \ldots \sigma_{k_{1}+k_{2}-1}}\right. \\
& \left.-\frac{1}{k_{1} !\left(k_{2}-1\right) !} \partial_{\lambda} K_{1}^{\sigma \sigma_{1} \ldots \sigma_{k_{1}}} K_{2}^{\sigma_{k_{1}+1} \ldots \sigma_{k_{1}+k_{2}-1}}\right) \\
& =-\frac{1}{\left(k_{1}+k_{2}-1\right) !} p_{\sigma_{1}} \ldots p_{\sigma_{k_{1}+k_{2}-1}}\left[K_{1}, K_{2}\right]^{\sigma_{1} \ldots \sigma_{k_{1}+k_{2}-1}} \\
& =-\mathcal{C}\left[\kappa_{1}, \kappa_{2}\right] \text {, }
\end{aligned}
$$

e usando (2.16), (2.12), (2.6), o fato de que o tensor métrico é covariantemente constante e, finalmente, (2.26), concluímos que

$$
\begin{aligned}
k ! \frac{d}{d \tau}\left(\mathcal{C}\left(K^{\top}\right)\right)= & \frac{d}{d \tau}\left(p_{\mu_{1}} \ldots p_{\mu_{k}} K^{\mu_{1} \ldots \mu_{k}}\right) \\
= & \frac{D p_{\mu_{1}}}{d \tau} p_{\mu_{2}} \ldots p_{\mu_{k}} K^{\mu_{1} \ldots \mu_{k}}+\ldots+p_{\mu_{1}} \ldots p_{\mu_{k-1}} \frac{D p_{\mu_{k}}}{d \tau} K^{\mu_{1} \ldots \mu_{k}} \\
& +p_{\mu_{1}} \ldots p_{\mu_{k}} \frac{D K^{\mu_{1} \ldots \mu_{k}}}{d \tau} \\
= & g_{\mu_{1} \lambda_{1}} \ldots \mathrm{g}_{\mu_{k} \lambda_{k}} \dot{q}^{\lambda_{1}} \ldots \dot{q}^{\lambda_{k}} \dot{q}^{\lambda} \nabla_{\lambda} K^{\mu_{1} \ldots \mu_{k}} \\
= & \dot{q}^{\lambda_{1}} \ldots \dot{q}^{\lambda_{k}} \dot{q}^{\lambda} \nabla_{\lambda} K_{\lambda_{1} \ldots \lambda_{k}} \\
= & 0 .
\end{aligned}
$$

Concluímos com algumas observações sobre uma possivel interpretação geométrica dos campos tensoriais de Killing. É um princípio geral da física que quantidades conservadas são sempre associadas a simetrias. Impõe-se, portanto, a questão de qual natureza seria a simetria gerada por um campo tensorial de Killing $k$ de grau $k>1$, pois ao contrário dos campos vetoriais de Killing, esse não gera um fluxo na variedade $Q$. Uma possível resposta consiste em considerar o campo Hamiltoniano $X_{\mathcal{C}\left(K^{\circ}\right)}$ : este sim gera um fluxo. mas 
na variedade $T^{*} Q$. Isso significa que, em geral. a simetria gerada por um campo tensorial de Killing $K$ reside no espaço de fase e não no espaço de configuração: somente no caso $k=1$ é possível projetar o fluxo de $\mathrm{X}_{\mathcal{C}(K)}$ em $T^{*} Q$ para $Q$, o que obviamente reproduz o fluxo de K em $Q$. 


\section{Capítulo 3}

\section{Relatividade Geral}

Na mecânica Newtoniana, como já comentamos, a dinâmica de uma partícula é descrita por uma trajetória $q: I \rightarrow Q$, onde o parâmetro $\tau$ é o tempo absoluto e a variedade $Q$ é o espaço no qual a partícula se move sob a influência das forças externas às quais está sujeita. segundo a equação de Newton. Estas forças incluem a força gravitacional, regicla pela lei gravitacional de Newton.

No início deste século, a formulação da teoria da Relatividade Restrita e da Relatividade Geral introduziu uma tranformação radical dessa visão. Primeiro, a Relatividade Restrita derrubou os conceitos Newtonianos do tempo e do espaço absoluto, que foram substituídos pelo conceito do espaço-tempo de Einstein e Minkowski. Logo, a dinâmica de uma partícula passou a ser descrita por uma trajetória $q: I \rightarrow Q$ onde o parâmetro $\tau$ é um parâmetro qualquer sem significado físico dado "a priori" e a variedade $Q$ é o espaço-tempo: foi introduzido o termo "linha de universo" para descrever esta nova noção relativística de trajetória. Segundo, a Relatividade Geral geometrizou a gravitação, no sentido de "absorver" as forças gravitacionais de Newton dentro da métrica do espaço-tempo. Em particular, a noçào Newtoniana de uma "partícula livre", i.e., uma partícula que não está sujeita a nenhuma força externa e portanto, segundo Galilei, se encontra em movimento retilíneo uniforme, é substituída pela noção relativística de uma "partícula em queda livre", i.e., uma partícula que não está sujeita a nenhuma força externa exceto a influência da gravitação, da qual nada escapa, e que, segundo o princípio de equivalência de Einstein, segue uma geodésica - a generalizaçào natural do movimento retilíneo uniforme a variedades curvas. Terceiro, a Relatividade Restrita estabeleceu a equivalência entre massa e energia, enquanto que a Relatividade Geral identificou estas como sendo não apenas sujeitas à influência da gravitação mas também como sendo sua fonte, através do tensor de energia-momento $T_{\mu \nu}$ que aparece no lado direito das equações de Einstein

$$
R_{\mu \nu}-\frac{1}{2} R \mathrm{~g}_{\mu \nu}=8 \pi T_{\mu \nu},
$$

onde $R_{\mu \nu}$ é o tensor de Ricci associado ao tensor métrico $\mathrm{g}_{\mu \nu}$ do espaço-tempo. Encontrar soluçòes exatas destas equaçòes é extremamente difícil. uma vez que estas constituem um 
sistema de equaçòes diferencias parciais não-lineares para o tensor métrico, que são acopladas com as equaçòes de evolução para a matéria que determinam a evolução do tensor de energiamomento. Porém, a presença de simetrias pode levar a simplificações drásticas, tornando factível o trabalho de encontrar soluções exatas.

\subsection{Soluções Exatas}

As soluções exatas das equações de Einstein que são conhecidas se dividem em duas grandes classes: as soluções cosmológicas e as soluções localizadas. Tipicamente, as primeiras são soluções com um tensor de energia-momento diferente de zero e com dependência não-trivial do tempo, descrevendo a distribuição da matéria no universo: as soluções mais conhecidas, de Friedmann e de Robertson-Walker, correspondem a um universo onde a matéria é descrita por um fluido ideal homogêneo e isotrópico. As segundas são soluções no vácuo, i.e., com tensor de energia-momento nulo. e são estáticas ou pelo menos estacionárias: as soluções mais conhecidas, de Schwarzschild e de Kerr, descrevem o campo gravitacional gerado por um corpo isolado, por exemplo uma estrela, na região externa. Em todos os casos, o conceito de simetrias tem um papel fundamental na obtenção dessas soluções, pois servem para reduzir substancialmente o número de variáveis independentes e dependentes das equações de Einstein.

Os tipos mais comuns de simetrias em variedades de Lorentz são os seguintes:

- espaços-tempos homogêneos;

- espaços-tempos isotrópicos;

- espaços-tempos estacionários;

- espaços-tempos estáticos;

- espaços-tempos esfericamente simétricos;

- espaços-tempos axialmente simétricos.

Os primeiros quatro tipos são importantes na classificação de soluções cosmológicas e os últimos quatro tipos na classificação de soluções localizadas. Como estamos interessados somente nesta última classe, vamos dar as definições apenas para os últimos quatro tipos.

Um espaço-tempo é dito estacionário se seu grupo de isometrias contém um subgrupo a um parâmetro cujas órbitas são curvas tipo tempo. Este subgrupo de isometrias expressa a simetria ou invariança do espaço-tempo por translação temporal e fornece um campo vetorial de Killing tipo tempo X. Denotando o parâmetro ao longo das curvas integrais de $X$ por $t$, podemos introduzir, em torno de cada ponto. coordenadas locais $x^{\mu}=\left(t, x^{i}\right)$ tal que a métrica assume a forma

$$
d s^{2}=-V^{2}\left(x^{1} \cdot x^{2}, x^{3}\right) d t^{2}+2 h_{i}\left(x^{1}, x^{2}, x^{3}\right) d t d x^{i}+h_{i j}\left(x^{1}, x^{2}, x^{3}\right) d x^{i} d x^{j},
$$


onde $V^{2}=g_{\mu \nu} \cdot X^{\mu} \cdot X^{\nu}$. e o campo vetorial de Killing assume a forma $X=\partial_{t}$. Ademais, quando este for completo, o que certamente será o caso quando o espaço-tempo for temporalmente geodesicamente completo, podemos afirmar que o parâmetro do seu fluxo providencia uma coordenada tempo global $t: Q \rightarrow \mathbb{R}$ sobre o espaço-tempo.

Um espaço-tempo é dito estático se ele for estacionário e se ele admitir uma folheação em hipersuperfícies tipo espaço que são ortogonais ao campo vetorial de Killing tipo tempo, providenciando assim uma decomposição natural do espaco-tempo em "espaço" e "tempo", que não existe em geral. Tal decomposição se reflete na existência de sistemas de coordenadas especiais: se $\Sigma$ é qualquer uma das hipersuperfícies da folheação dada, então existe uma vizinhança aberta de $\Sigma$ tal que todos os pontos do espaço-tempo nela contidos pertencem a uma única curva integral do campo vetorial de Killing $X$ que passa por $\Sigma$. Assim podemos construir, a partir de um sistema arbitrário de coordenadas locais $x^{i}$ de $\Sigma$, um sistema de coordenadas locais $\left(x^{\mu}\right)=\left(t, x^{i}\right)$ de $Q$, onde $t$ é a coordenada que, a cada ponto $q$ na referida vizinhança de $\Sigma$, associa o valor do parâmetro ao longo da curva integral de $X$ que começa em $\Sigma$ e termina em q. Em tais coordenadas locais, a métrica assume a forma

$$
d s^{2}=-V^{2}\left(x^{1}, x^{2}, x^{3}\right) d t^{2}+h_{i j}\left(x^{1}, x^{2}, x^{3}\right) d x^{i} d x^{j},
$$

onde $V^{2}=g_{\mu \nu} X^{\mu} X^{\nu}$, e o campo vetorial de Killing assume a forma $X=\partial_{t}$. A ausência de termos cruzados $d t d x^{i}$ em (3.3) expressa a ortogonalidade entre as hipersuperfícies da folheação e o campo vetorial de Killing. Ademais, quando este for completo, as hipersuperfícies da folheação são simplesmente as hipersuperfícies de nível da coordenada tempo global $t: \Sigma_{t_{0}}=\left\{q \in Q / t(q)=t_{0}\right\}$.

Um espaço-tempo é dito esfericamente simétrico se seu grupo de isometrias contém um subgrupo isomorfo ao grupo $S O(3)$ de rotações cujas órbitas são subvariedades tipo espaço do espaço-tempo e sào esferas bidimensionais. Este subgrupo de isometrias expressa a simetria ou invariança do espaço-tempo por rotações em torno de um "ponto" (as aspas indicam que tal "ponto" pode nào existir dentro do espaço-tempo considerado) e fornece três campos de Killing tipo espaço $X_{i}^{L}(i=1,2,3)$ que, com respeito ao colchete de Lie, satisfazem as regras de comutação da álgebra de Lie $\mathfrak{s o ( 3 )}$ (a menos de um sinal):

$$
\left[X_{i}^{L}, X_{j}^{L}\right]=-\epsilon_{i j k} \cdot X_{k}^{L} .
$$

Ademais, em cada órbita do espaço-tempo sob a ação de $S O(3)$, a métrica do espaço-tempo induz uma métrica que deve ser um múltiplo da métrica padrão da 2-esfera unitária e portanto poderá ser caracterizada pela área total $A$ da órbita, sendo conveniente introduzir o raio $r$ da órbita como a funçào definida por

$$
r=\left(\frac{A}{4 \pi}\right)^{1 / 2} .
$$

Assim, usando coordenadas esféricas $(\vartheta, \uparrow)$ para a 2-esfera unitária. a métrica em cada uma destas órbitas assume a forma

$$
d s^{2}=r^{2}\left(d \vartheta^{2}+\sin ^{2} \vartheta d \varphi^{2}\right) .
$$


Um espaço-tempo é dito axialmente simétrico se seu grupo de isometrias contém um subgrupo isomorfo ao grupo $S O(2)$ de rotaçòes cujas órbitas são subvariedades tipo espaço do espaço-tempo e são círculos. Este subgrupo de isometrias expressa a simetria ou invariança do espaço-tempo por rotações em torno de um "eixo" (as aspas indicam que tal "eixo" pode nào existir dentro do espaço-tempo considerado) e fornece um campo de Killing tipo espaço $X^{L}$. Ademais, em cada órbita do espaço-tempo sob a ação de $S O(2)$, a métrica do espaço-tempo induz uma métrica que deve ser um múltiplo da métrica padrão do círculo unitário e portanto poderá ser caracterizada pela circunferência total $l$ da órbita, sendo conveniente introduzir o raio $\rho$ da órbita como a função definida por

$$
\rho=\frac{l}{2 \pi} \text {. }
$$

Assim, usando a coordenada angular y para o círculo unitário, a métrica em cada uma destas órbitas assume a forma

$$
d s^{2}=\rho^{2} d \varphi^{2} .
$$

Finalmente, observamos que um espaço-tempo é considerado estático e esfericamente simétrico se é simultaneamente estático e esfericamente simétrico $e$ se as açòes do grupo de translação temporal e do grupo de rotações espaciais comutam. Combinando os sistemas de coordenadas introduzidos acima, podemos concluir que, neste caso, a métrica assume a forma

$$
d s^{2}=-f^{2}(r) d t^{2}+g^{2}(r) d r^{2}+h^{2}(r) r^{2}\left(d \vartheta^{2}+\sin ^{2} v d \varphi^{2}\right),
$$

com $h \equiv 1$ se $r$ for a variável raio introduzicla anteriormente. O exemplo mais simples é o espaço de Minkowski, cuja métrica, nestas coordenadas, assume a forma

$$
d s^{2}=-d t^{2}+d r^{2}+r^{2}\left(d \vartheta^{2}+\sin ^{2} v d \varphi^{2}\right) .
$$

\subsection{Solução de Schwarzschild}

Em 1916, pouco tempo após ter divulgado seus trabalhos fundamentais sobre Relatividade Geral, Einstein recebeu em Berlim uma carta de um astrônomo que estava lutando na Rússia pelo exército alemão. Karl Schwarzschild. Apesar de estar hospitalizado com um ferimento que lhe seria fatal, Schwarzschild ainda teve o tempo de descobrir uma soluçào das equações de Einstein no vácuo, i.e., com tensor de energia-momento nulo, descrevendo o campo gravitacional gerado por um corpo isolado em repouso, sem movimentos internos e, em particular, sem rotação. Esta solução, hoje conhecida como a solução de Schwarzschild, é estática e esfericamente simétrica. De fato, restringindo-se a métricas da forma (3.9), com $h \equiv 1$, e resolvendo as equações de Einstein no vácuo, obtem-se a métrica de Śchwarzschild em coordenadas esféricas $(t, r, \vartheta, \varphi)$,

$$
d s^{2}=-\left(1-\frac{2 m}{r}\right) d t^{2}+\left(1-\frac{2 m}{r}\right)^{-1} d r^{2}+r^{2}\left(d v^{2}+\sin ^{2} v d \varphi^{2}\right)
$$


onde $m$ é um parâmetro real. com $m>0$ para garantir que, no limite Newtoniano, as forças gravitacionais sejam atrativas e não repulsivas ${ }^{1}$, e as variáveis $t, \vartheta$ e $\varphi$ percorrem os domínios

$$
-\infty<t<\infty \quad, \quad 0<\vartheta<\pi \quad, \quad 0<\varphi<2 \pi \text { ou }-\pi<\varphi<\pi
$$

Os valores $\varphi=0$ e $\varphi=2 \pi$ ou $\varphi= \pm \pi$ (meridianos), $\vartheta=0$ (pólo norte) e $\vartheta=\pi$ (pólo sul) caracterizam pontos no espaço-tempo onde o sistema de coordenadas $(t, r, \vartheta, \hat{\varphi})$ falha, mas este tipo de singularidade de coordenadas é bem conhecida da geometria do espaço Euclideano $\mathbb{R}^{3}$ : o problema é facilmente resolvido entendendo (3.11) como definindo a métrica de Schwarzschild num subconjunto aberto e denso da variedade $\mathbb{R}^{2} \times S^{2}$, definido pelas condições $r \neq 0$ e $r \neq 2 m$.

A questão sobre qual seria o domínio admissivel para a variável radial $r$ é mais delicada. Primeiramente. observa-se que alguns dos componentes do tensor métrico são singulares quando $r=0$ ou $r=2 \mathrm{~m}$. A condição $r=0$ caracteriza uma verdadeira singularidade do espaço-tempo. como pode ser demonstrado, por exemplo, pela observação de que o traço do quadrado do tensor de curvatura diverge no limite $r \rightarrow 0$ [12, pp. 100-102]. Esta singularidade tem a topologia de uma linha no espaço-tempo (o que corresponde a um ponto no espaço) e portanto é chamada a singularidade central. A condição $r=2 m$, por outro lado, divide o espaço-tempo em dois componentes distintos:

$$
\begin{aligned}
& \text { Bloco I - Região externa ou exterior : } 2 m<r<\infty \text {, } \\
& \text { Bloco II - Região interna ou interior : } 0<r<2 m .
\end{aligned}
$$

Ao contrário da singularidade de curvatura localizada em $r=0$, a condição $r=2 m$ caracteriza apenas uma singularidade de coordenadas que pode ser eliminada através de uma transformação de coordenadas (coordenadas de Eddington-Finkelstein e de Kiruskal), identificando os blocos I e II como partes de uma variedade maior, chamada a extensão de Kiruskal do espaço-tempo de Schwarzschild [8, pp. 149-156], [19, pp. 148-156]. Nesta extensão, cada dois blocos são colados por uma subvariedade que corresponde à condição $r=2 m$ e, constituindo a fronteira comum entre dois blocos, caracteriza o limite de validade, ou seja, o horizonte do sistema de coordenadas de cada um. Esses horizontes possuem um significado invariante, i.e., independente da escolha de coordenadas, pois cada um deles age como um tipo de membrana semi-permeável no sentido que toda geodésica, assim como qualquer outra trajetória tipo tempo ou tipo luz, só pode atravessá-la em uma determinada direção.

Passando à discussão dos campos de Kïlling, observamos inicialmente que a solução de Schwarzschild possue dois campos vetoriais de Killing óbvios, que são os campos vetoriais $\partial_{t}$ e $\partial_{\varphi}$ associados às coordenadas $t$ e $\varphi$, já que os componentes do tensor métrico (3.11) não dependem de $t$ ou de $\varphi$. (Nota-se, de passagem, que o campo vetorial $\partial_{t}$ é tipo tempo apenas no bloco I mas é tipo espaço no bloco II. o que significa que na região interna a métrica de Schwarzschild descreve um espaço-tempo que não é estático. nem estacionário.) Juntos

\footnotetext{
${ }^{1}$ Observemos que ao tomarmos o valor $m=0$, obtemos a métrica de Minkowski.
} 
com o tensor métrico. eles definem três quantidades conservadas para o fluxo geodésico que estão em involução, que são a Hamiltoniana $H$, a energia $E$ e o 3-componente do momento angular $L_{3}$. Usando a liberdade de reparametrização afim de geodésicas, podemos normalizar o parâmetro afim de tal maneira que $-2 H$ vale 1 para geodésicas tipo tempo, enquanto que vale 0 para geodésicas tipo luz. De qualquer modo, em termos de coordenadas em $T Q$,

$$
\begin{gathered}
E=-\mathrm{g}_{\mu \nu}\left(\partial_{t}\right)^{\mu} \dot{q}^{\nu}=\left(1-\frac{2 m}{r}\right) \dot{t} \\
L_{3}=\mathrm{g}_{\mu \nu}\left(\partial_{\varphi}\right)^{\mu} \dot{q}^{\nu}=r^{2} \sin ^{2} \vartheta \dot{\varphi},
\end{gathered}
$$

$\mathrm{e}$

$$
2 H=\mathrm{g}_{\mu \nu} \dot{q}^{\mu} \dot{q}^{\nu}=-\left(1-\frac{2 m}{r}\right) \dot{t}^{2}+\left(1-\frac{2 m}{r}\right)^{-1} \dot{r}^{2}+r^{2} \dot{\vartheta}^{2}+r^{2} \sin ^{2} \vartheta \dot{\varphi}^{2} .
$$

Como o espaço-tempo de Schwarzschild é esfericamente simétrico e não apenas axialmente simétrico, temos além de $\partial_{t}$ três outros campos vetoriais de Killing, $X_{1}^{L}, X_{2}^{L}$ e $X_{3}^{L}$, que correpondem aos três componentes do momento angular. $L_{1}, L_{2}$ e $L_{3}$, dados por

$$
\begin{gathered}
X_{1}^{L}=-\sin \varphi \partial_{\vartheta}-\cot \vartheta \cos \varphi \partial_{\varphi}, \\
X_{2}^{L}=\cos \varphi \partial_{\vartheta}-\cot \vartheta \sin \varphi \partial_{\varphi}, \\
X_{3}^{L}=\partial_{\varphi},
\end{gathered}
$$

e, em termos de coordenadas em $T Q$,

$$
\begin{gathered}
L_{1}=\mathrm{g}_{\mu \nu}\left(X_{1}^{L}\right)^{\mu} \dot{q}^{\nu}=-r^{2} \sin \varphi \dot{v}-r^{2} \sin \vartheta \cos \vartheta \cos \varphi \dot{\varphi}, \\
L_{2}=\mathrm{g}_{\mu \nu}\left(X_{2}^{L}\right)^{\mu} \dot{q}^{\nu}=r^{2} \cos \varphi \dot{v}-r^{2} \sin \vartheta \cos \vartheta \sin \varphi \dot{\varphi}, \\
L_{3}=\mathrm{g}_{\mu \nu}\left(X_{3}^{L}\right)^{\mu} \dot{q}^{\nu}=r^{2} \sin ^{2} \vartheta \dot{\varphi} .
\end{gathered}
$$

Também existe, além do tensor métrico, um outro campo tensorial de Killing de grau $2, K$ : ele corresponde ao quadrado do momento angular, ou melhor, à soma $L^{2}=L_{1}^{2}+L_{2}^{2}+L_{3}^{2}$ dos quadrados dos componentes do momento angular. $\mathrm{Na}$ forma contravariante, temos $K=\left(X_{1}^{L}\right)^{2}+\left(X_{2}^{L}\right)^{2}+\left(X_{3}^{L}\right)^{2}$, i.e.,

$$
K=\partial_{\vartheta}^{2}+\frac{1}{\sin ^{2} \vartheta} \partial_{\vartheta}^{2}
$$

e na forma covariante

$$
K=r^{4}\left(d v^{2}+\sin ^{2} \vartheta d \vartheta^{2}\right) .
$$


Assim a quantidade conservada correspondente, em termos de coordenadas em $T Q$, é

$$
L^{2}=r^{4} \dot{\vartheta}^{2}+r^{4} \sin ^{2} \vartheta \dot{\varphi}^{2} .
$$

De fato, como o colchete de Schouten é uma derivação em cada um dos seus argumentos, com respeito ao produto tensorial simetrizado, qualquer polinômio em campos vetoriais (ou tensoriais) de Killing é novamente um campo tensorial de Killing. A vantagem do campo $K$ é que ele fornece uma quarta quantidade conservada para o fluxo geodésico que está em involução com as demais, pois obviamente o colchete de Schouten de $K$ com $X_{3}^{L}=\partial_{\varphi}$ se anula, o que não é verdade para $X_{1}^{L}$ ou $X_{2}^{L}$. Ademais, as quantidades conservadas $E, L_{3}$, $L^{2}$ e $H$ são funcionalmente independentes, como pode ser verificado através de um cálculo explícito do posto da matriz Jacobiana

$$
\begin{aligned}
& \frac{\partial\left(E, L_{3}, L^{2}, 2 H\right)}{\partial(t, r, \vartheta, \varphi, \dot{t}, \dot{r}, \dot{v}, \dot{\varphi})}=\left(\begin{array}{cccccccc}
\partial_{t} E & \partial_{r} E & \partial_{\vartheta} E & \partial_{\varphi} E & \partial_{\dot{t}} E & \partial_{\dot{r}} E & \partial_{\dot{\vartheta}} E & \partial_{\dot{\varphi}} E \\
\partial_{t} L_{3} & \partial_{r} L_{3} & \partial_{\vartheta} L_{3} & \partial_{\varphi} L_{3} & \partial_{\dot{t}} L_{3} & \partial_{\dot{r}} L_{3} & \partial_{\dot{\vartheta}} L_{3} & \partial_{\dot{\varphi}} L_{3} \\
\dot{\partial}_{t} L^{2} & \partial_{r} L^{2} & \partial_{\vartheta} L^{2} & \partial_{\varphi} L^{2} & \partial_{\dot{t}} L^{2} & \partial_{\dot{r}} L^{2} & \partial_{\dot{\vartheta}} L^{2} & \partial_{\dot{\varphi}} L^{2} \\
2 \partial_{t} H & 2 \partial_{r} H & 2 \partial_{\vartheta} H & 2 \partial_{\varphi} H & 2 \partial_{\dot{t}} H & 2 \partial_{\dot{r}} H & 2 \partial_{\dot{\vartheta}} H & 2 \partial_{\dot{\varphi}} H
\end{array}\right) \\
& =\left(\begin{array}{cccc}
0 & 2 m E / r^{2}(1-2 m / r)^{-1} & 0 & 0 \\
0 & 2 L_{3} / r & 2 \cos \vartheta L_{3} / \sin \vartheta & 0 \\
0 & 4 L^{2} / r & 2 \cos \vartheta L_{3}^{2} / \sin ^{3} \vartheta & 0 \\
0 & 2 \partial_{r} H & 2 \cos \vartheta L_{3}^{2} / r^{2} \sin ^{3} \vartheta & 0
\end{array}\right. \\
& \left.\begin{array}{cccc}
1-2 m / r & 0 & 0 & 0 \\
0 & 0 & 0 & r^{2} \sin ^{2} \vartheta \\
0 & 0 & 2 r^{4} \dot{\vartheta} & 2 r^{2} L_{3} \\
-2 E & 2(1-2 m / r)^{-1} \dot{r} & 2 r^{2} \dot{\vartheta} & 2 L_{3}
\end{array}\right)
\end{aligned}
$$

O subdeterminante da derivada em relação às velocidades é

$$
\begin{aligned}
\operatorname{det}\left(\frac{\partial\left(E, L_{3}, L^{2}, 2 H\right)}{\partial(\dot{t}, \dot{r}, \dot{\vartheta}, \dot{\varphi})}\right) & =\operatorname{det}\left(\begin{array}{cccc}
1-2 m / r & 0 & 0 & 0 \\
0 & 0 & 0 & r^{2} \sin ^{2} \vartheta \\
0 & 0 & 2 r^{4} \dot{\vartheta} & 2 r^{2} L_{3} \\
-2 E & 2(1-2 m / r)^{-1} \dot{r} & 2 r^{2} \dot{\vartheta} & 2 L_{3}
\end{array}\right) \\
& =2(1-2 m / r)^{-1} \dot{r} \operatorname{det}\left(\begin{array}{ccc}
1-2 m / r & 0 & 0 \\
0 & 0 & r^{2} \sin ^{2} \vartheta \\
0 & 2 r^{4} \dot{\vartheta} & 2 r^{2} L_{3}
\end{array}\right) \\
& =2 \dot{r} \operatorname{det}\left(\begin{array}{cc}
0 & r^{2} \sin ^{2} \vartheta \\
2 r^{4} \dot{\vartheta} & 2 r^{2} L_{3}
\end{array}\right) \\
& =-4 r^{6} \sin ^{2} \vartheta \dot{r} \dot{v} .
\end{aligned}
$$

Definindo

$$
Z=Z_{\sin \vartheta} \cup Z_{\dot{\vartheta}} \cup Z_{\dot{r}},
$$


onde

$$
\begin{aligned}
Z_{\sin \vartheta} & =\{(q, \dot{q}) / \sin \vartheta=0\} \\
Z_{\dot{\vartheta}} & =\{(q, \dot{q}) / \dot{\vartheta}=0\}, \\
Z_{\dot{r}} & =\{(q, \dot{q}) / \dot{r}=0\} .
\end{aligned}
$$

isso mostra que os gradientes das quantidades conservadas são linearmente independentes fora de $Z$, e como $Z$ é um subconjunto de medida nula no espaço de fase $T Q$, concluímos que as quantidades conservadas são funcionalmente independentes. (Na verdade, calculando outros subdeterminantes, podemos mostrar que o subconjunto de dependência funcional é ainda menor do que o $Z$ dado aqui, mas não precisaremos deste fato.) Resumindo, podemos concluir que o fluxo geodésico na solução de Schwarzschild é um sistema Hamiltoniano completamente integrável.

Na discussão da solução de Schwarzschild em livros texto sobre Relatividade Geral, a quantidade $L^{2}$ nào é normalmente considerada, o que pode parecer até estranho tendo em vista a grande importância que ela tem em outras áreas da física como, por exemplo, na mecânica quântica. Ao invés disso, é costume reduzir o número de graus de liberdade do sistema usando o fato de que a conservação da direção do vetor do momento angular significa que o movimento geodésico na solução de Schwarzschild é planar: cada geodésica permanece no plano gerado por sua posição inicial e pelo vetor de velocidade inicial, além do centro de atração. Escolhendo as coordenadas esféricas de tal forma que este plano seja o plano equatorial caracterizado pela condiçào $\vartheta=\pi / 2$, a variável $\vartheta$ acaba sendo elimina.da, pois $\dot{y}=0$. No entanto, como o mencionado plano depende das condições iniciais, este procedimento não parece adequado para um tratamento simultâneo de famílias de geodésicas.

De qualquer forma, podemos expressar, por exemplo, $\dot{r}$ exclusivamente em termos de $r$ e de quantidades conservadas:

$$
\dot{r}^{2}=E^{2}-\left(1-\frac{2 m}{r}\right)\left(\frac{L^{2}}{r^{2}}-2 H\right)
$$

Esta equaçào mostra que o movimento radial de uma geodésica coincide com o movimento, no contexto da mecânica Newtoniana, de uma partícula de massa unitária com energia $\frac{1}{2} E^{2}+H$ dentro de um potencial efetivo

$$
V_{\mathrm{eff}}(r)=\frac{2 H m}{r}+\frac{L^{2}}{2 r^{2}}-\frac{m L^{2}}{r^{3}} .
$$

A diferença crucial ao problema de Kepler é que, em adição ao potencial de Newton/Coulomb $2 \mathrm{Hm} / \mathrm{r}$ (igual a $-m / r$ para geodésicas tipo tempo e igual a 0 para geodésicas tipo luz) e ao termo repulsivo $L^{2} / 2 r^{2}$ que representa a "barreira centrifugal", temos um termo novo $-m L^{2} / r^{3}$ que, para pequenos valores de $r$, domina os demais termos e é responsável por fenômenos tais como a defleção da luz perto do sol e a rotação do perihélio de Mercúrio. Observe que é através desta análise comparativa que podemos concluir que o parâmetro $m$ na métrica de Schwarzschild (3.11) deve ser positivo, para garantir que o potencial de Newton/Coulomb represente uma força atrativa e não repulsiva. 


\subsection{Solução de Kerr}

Em 1963, o físico neozelandês Roy Kierr encontrou uma nova solução das equações de Einstein no vácuo, i.e., com tensor de energia-momento nulo, descrevendo o campo gravitacional gerado por um corpo isolado em repouso, sem movimentos internos, exceto por rotação em torno de um eixo fixo e com velocidade angular constante. Esta solução, hoje conhecida como a solução de Kierr, é estacionária e axialmente simétrica. Uma solução desta natureza havia sido procurada pelos físicos, sem sucesso, durante quase 50 anos e finalmente foi descoberta de maneira indireta, quando empregou-se a classificaçào de Petrov das variedades de Einstein desenvolvida durante a década dos '50. Posteriormente, em 1967, Boyer e Lindquist apresentaram a forma explícita da métrica de $\operatorname{Kerr}$ em coordenadas $(t, r, \vartheta, \varphi)$, que no contexto desta geometria sào chamadas as coordenadas de Boyer-Lindquist:

$$
\begin{aligned}
d s^{2}= & -\left(1-\frac{2 m r}{\rho^{2}}\right) d t^{2}+\frac{\rho^{2}}{\lrcorner} d r^{2}+\rho^{2} d \vartheta^{2} \\
& +\left(r^{2}+a^{2}+\frac{2 m a^{2} r \sin ^{2} \vartheta}{\rho^{2}}\right) \sin ^{2} \vartheta d \varphi^{2}-\frac{2 m a r \sin ^{2} \vartheta}{\rho^{2}} 2 d t d \varphi,
\end{aligned}
$$

com

$$
\begin{aligned}
& \rho^{2}=r^{2}+a^{2} \cos ^{2} \vartheta, \\
& \lrcorner=r^{2}-2 m r+a^{2},
\end{aligned}
$$

onde $m$ e a são parâmetros reais, com $m>0$ para garantir que, no limite Newtoniano, as forças gravitacionais sejam atrativas e não repulsivas, e as variáveis $t$, $\vartheta$ e $\varphi$ percorrem os domínios

$$
-\infty<t<\infty \quad, \quad 0<\vartheta<\pi \quad, \quad 0<\varphi<2 \pi \text { ou }-\pi<\varphi<\pi .
$$

Os valores $\varphi=0$ e $\varphi=2 \pi$ ou $\varphi= \pm \pi$ (meridianos), $\vartheta=0$ (pólo norte) e $\vartheta=\pi$ (pólo sul) caracterizam pontos no espaço-tempo onde o sistema de coordenadas $(t, r, \vartheta, \varphi)$ falha, mas este tipo de singularidade de coordenadas é bem conhecida da geometria do espaço Euclideano $\mathbb{R}^{3}$ : o problema é facilmente resolvido entendendo (3.24) como definindo a métrica de Kerr num subconjunto aberto e denso da variedade $\mathbb{R}^{2} \times S^{2}$, definido pelas condições $\rho \neq 0$ e $\Delta \neq 0$.

A questão sobre qual seria o domínio admissível para a variável radial $r$ é mais delicada. Primeiramente, observa-se que alguns dos componentes do tensor métrico são singulares quando $\rho=0$ ou $\Delta=0$. A condiçào $\rho=0$, que é equivalente às duas condições $r=0 \mathrm{e}$ $\vartheta=\pi / 2$, caracteriza uma verdadeira singularidade do espaço-tempo, como pode ser demonstrado, por exemplo, pela observaçào de que o traço do quadrado do tensor de curvatura diverge no limite $r \rightarrow 0$ [12. pp. 100-102]. Contrariamente à primeira expectativa, esta singularidade tem a topologia de um cilindro no espaço-tempo (o que corresponderia a um círculo no espaço, localizado no plano equatorial) e portanto é chamada a singularidade anel, sendo denotada aqui por $\Sigma$. A condição $\Delta=0$, por outro lado, requer uma distinção de casos, pois $\Delta$ é uma função quadrática de $r$ e pode ter duas. uma ou nenhuma raíz real. 
Inicialmente, observemos que a métrica de Kerr se reduz à métrica de Schwarzschild quando $a=0$, o que sugere interpretar a como o momento angular (ou uma função do momento angular) da fonte do campo gravitacional. Portanto, pequenos valores de $a$ correspondem a rotação lenta e grandes valores de a correspondem a rotação rápida, o que justifica. a seguinte terminologia:

Definição 3.1 As métricas de Kierr são divididas nas seguintes classes:

$$
\begin{array}{cl}
a^{2}=0 & \text { espaço-tempo de Schwarzschild, } \\
0<a^{2}<m^{2} & \text { espaço-tempo de Kerr lento } \\
a^{2}=m^{2} & \text { espaço-tempo de Kerr extremo, } \\
a^{2}>m^{2} & \text { espaço-tempo de Kerr rápido. }
\end{array}
$$

Obviamente. no caso da solução de Schwarzschild, a função $\Delta$ tem duas raízes reais, dadas por

$$
r_{+}=2 m, r_{-}=0 \quad \text { quando } a=0,
$$

e mais geralmente, no caso da soluçào de Kierr lenta, a função $\triangle$ continua tendo duas raízes reais, dadas por

$$
r_{ \pm}=m \pm \sqrt{m^{2}-a^{2}} \text {. }
$$

Também é claro que no caso da solução de lierr extrema, temos apenas uma raíz (dupla),

$$
r_{0}=m
$$

enquanto que no caso da soluçào de Kierr rápida, não existe nenhuma raíz real. Porém, estes últimos dois casos não descrevem situações físicas reais pois, descle o início. a matéria que constituiria a fonte do campo gravitacional a ser descrito pela solução de Kierr não se aglomera dentro de uma região limitada. mas tende a se espalhar numa região de extensão maior. quando o momento angular excede a massa total. Portanto, restringiremo-nos no que segue ao estudo da solução de Kerr lenta. Mesmo neste caso, observa-se que, ao contrário do que acontece na soluçào de Schwarzschild, a variável $r$ pode tomar o valor 0 e passar a assumir valores negativos, desde que $a \neq 0$ e desde que estejamos fora do plano equatorial, dado por $\vartheta=\pi / 2$. Assim, a condiçào $\lrcorner \neq 0$ divide o espaço-tempo em três componentes distintos, conhecidos como os blocos de Boyer-Lindquist e definidos por [12, p. 65]

$$
\begin{array}{lcc}
\text { Bloco } & \text { I }: r_{+}<r<\infty \quad ; \text { aqui, } \Delta>0, \epsilon=+1, \\
\text { Bloco } & \text { II }: r_{-}<r<r_{+} ; \text {aqui, } \Delta<0, \epsilon=-1, \\
\text { Bloco } \text { III }:-\infty<r<r_{-} ; \text {aqui, } \Delta>0, \epsilon=+1,
\end{array}
$$

onde introduzimos a abreviação

$$
\epsilon=\operatorname{sign}\lrcorner \text {. }
$$




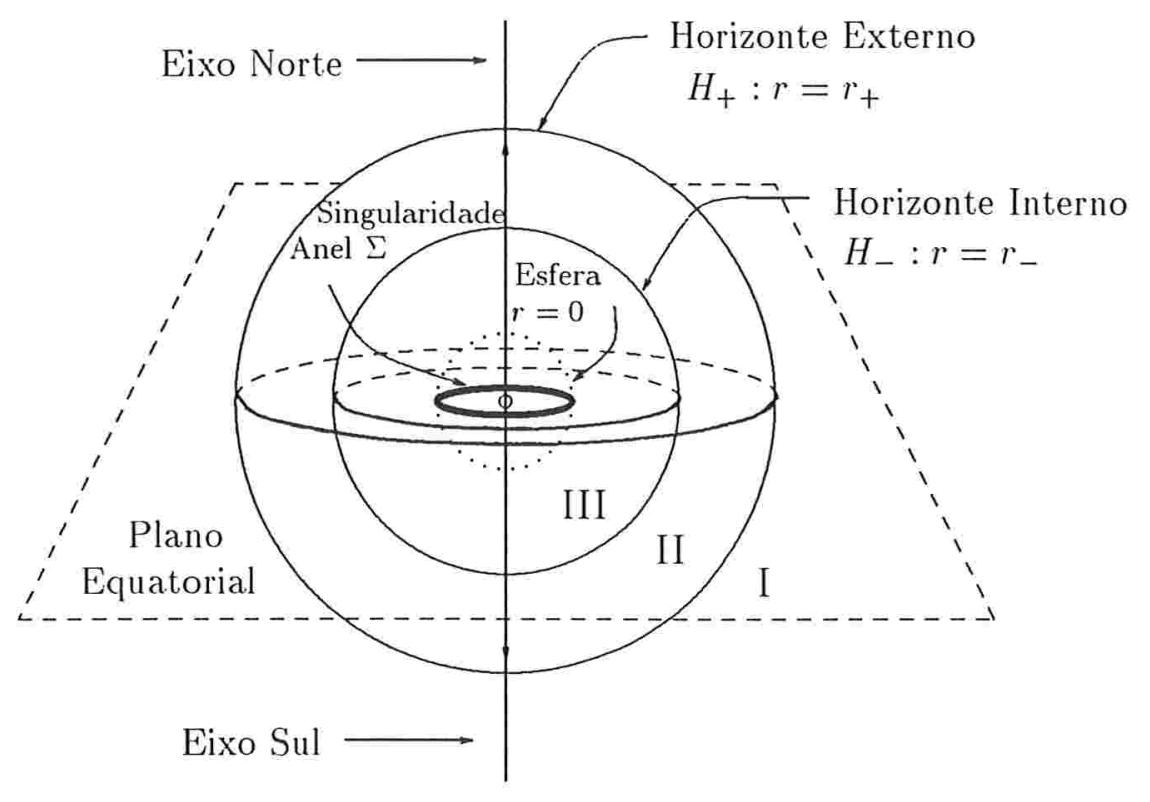

Figura 3.1. O espaço-tempo de Kerr lento. A figura representa uma fatia $t=$ const da variedade $\mathbb{R}^{2} \times S^{2}$, onde a variável $r(-\infty<r<\infty)$ está desenhada como $e^{r}$ $\left(0<\epsilon^{r}<\infty\right)$ : portanto, o centro da figura representa a região assintótica onde $r \rightarrow-\infty$ que, apesar de aparecer menor, é tào extensa quanto a região assintótica onde $r \rightarrow \infty$. O eixo de rotação consiste de duas retas completas: um eixo norte $(\vartheta=0)$ onde $z \equiv r \cos \vartheta=r(-\infty<z<\infty)$ e um eixo sul $(\vartheta=\pi)$ onde $z \equiv r \cos \vartheta=-r$ $(-\infty<z<\infty)$. A singularidade anel $\Sigma$ é formada pela interseçào da 2-esfera $r=0$ com o plano equatorial $\vartheta=\pi / 2$, mas nào obstrue a passagem entre as regiōes $r>0$ e $r<0$. Finalmente, cabe observar que o bloco II não é uma região espacial, pois neste bloco, a variável $r$ mede tempo e não distância.

Ao contrário da singularidade de curvatura localizada em $\rho=0$, a condição $\Delta=0$ caracteriza apenas uma singularidade de coordenadas que pode ser eliminada através de uma transformação de coordenadas (coordenadas de Kiruskal-Boyer-Lindquist), identificando os blocos I, II e III como partes de uma variedade maior, chamada a extensão maximal do espaço-tempo de Kierr lento, cuja estrutura é de grande complexidade [8, pp. 161-168]. [12, pp. 105-176]. Novamente, nesta extensào, cada dois blocos são colados por uma subvariedade que corresponde a uma das condições $r=r_{+}$ou $r=r_{-}$e, constituindo a fronteira comum entre dois blocos, caracteriza o limite de validade. ou seja, o horizonte do sistema de coordenadas de cada um. Esses horizontes possuem um significado invariante. i.e., independente da escolha de coordenadas, pois cada um deles age como um tipo de membrana semi-permeável no sentido que toda geodésica, assim como qualquer outra trajetória tipo tempo ou tipo luz, só pode atravessá-la em uma determinada direção. 
O fato de que a solução de Kerr é estacionária mas nào é estática expressa-se pela presença do termo cruzado $d t d \varphi$ em (3.24): os campos vetoriais coordenada $\partial_{t}$ e $\partial_{\varphi}$ não são ortogonais. Portanto, para uso posterior, introduzimos os campos vetoriais $[12$, p. 60$]$

$$
V=\left(r^{2}+a^{2}\right) \partial_{t}+a \partial_{\varphi}, W=\partial_{\varphi}+a \sin ^{2} \vartheta \partial_{t}
$$

que são ortogonais e satisfazem

$$
\mathrm{g}(V, V)=-\Delta \rho^{2}, \mathrm{~g}(W, W)=\rho^{2} \sin ^{2} \vartheta .
$$

Passando à discussão dos campos de Killing, observamos inicialmente que, novamente, a solução de Kerr possue dois campos vetoriais de Killing óbvios, que são os campos vetoriais $\partial_{t}$ e $\partial_{\varphi}$ associados às coordenadas $t$ e $\varphi$, já que os componentes do tensor métrico (3.24) não dependem de $t$ ou de $\varphi$. Juntos com o tensor métrico, eles definem três quantidades conservadas para o fluxo geodésico que estão em involução, que são a Hamiltoniana $H$, a energia $E$ e o momento angular $L_{3}$ em torno do eixo de rotação. Usando a liberdade de reparametrização afim de geodésicas, podemos normalizar o parâmetro afim de tal maneira que $-2 H$ vale 1 para geodésicas tipo tempo, enquanto que vale 0 para geodésicas tipo luz. De qualquer modo, em termos de coordenadas em $T Q$,

$$
\begin{gathered}
E=-\mathrm{g}_{\mu \nu}\left(\partial_{t}\right)^{\mu} \dot{q}^{\nu}=\left(1-\frac{2 m r}{\rho^{2}}\right) \dot{t}+\frac{2 m a r \sin ^{2} \vartheta}{\rho^{2}} \dot{\varphi} \\
L_{3}=\mathrm{g}_{\mu \nu}\left(\partial_{\varphi}\right)^{\mu} \dot{q}^{\nu}=\left(r^{2}+a^{2}+\frac{2 m a^{2} r \sin ^{2} \vartheta}{\rho^{2}}\right) \sin ^{2} \vartheta \dot{\varphi}-\frac{2 m a r \sin ^{2} \vartheta}{\rho^{2}} \dot{t}
\end{gathered}
$$

e

$$
\begin{aligned}
2 H=\mathrm{g}_{\mu \nu} \dot{q}^{\mu} \dot{q}^{\nu}= & -\left(1-\frac{2 m r}{\rho^{2}}\right) \dot{t}^{2}+\frac{\rho^{2}}{\Delta} \dot{r}^{2}+\rho^{2} \dot{\vartheta}^{2} \\
& +\left(r^{2}+a^{2}+\frac{2 m a^{2} r \sin ^{2} \vartheta}{\rho^{2}}\right) \sin ^{2} \vartheta \dot{\varphi}^{2}-\frac{2 m a r \sin ^{2} \vartheta}{\rho^{2}} 2 \dot{t} \dot{\varphi} .
\end{aligned}
$$

Como o espaço-tempo de Kierr é apenas axialmente simétrico, nào há motivo para esperar que existam outras quantidades conservadas, além das já mencionadas. Portanto, a descoberta de Carter [2] de uma constante de movimento adicional para o fluxo geodésico na solução de Kierr, hoje conhecida como a constante de Carter, foi geralmente recebida com surpresa. Posteriormente, Walker e Penrose [20] mostraram que a constante de Carter é o resultado da existência de um segundo campo tensorial de Killing $K$ que chamaremos tensor de WalkerPenrose e que, novamente, fornece uma quarta quantidade conservada para o fluxo geodésico.

A contribuição da presente dissertação é a demonstração do fato que a constante de Carter, considerada como a quarta quantidade conservada para o fluxo geodésico na soluçào de Kierr, está em involução com as demais, pois o colchete de Schouten do tensor de WalkerPenrose com o tensor métrico e com os dois campos vetoriais de Killing $\partial_{t}$ e $\partial_{\varphi}$ é zero. 
Ademais, mostraremos que as quantidades conservadas assim obtidas são funcionalmente independentes, o que permite concluir que o fluxo geodésico na soluçào de Kerr é um sistema Hamiltoniano completamente integrável.

Iniciaremos a demonstraçào dessas afirmações por uma breve apresentação de um método relativamente direto para se chegar à constante de Carter, seguindo [12, p. 60].

Considerando a expansão do campo vetorial tangente $\dot{\gamma}$ à uma geodésica $\gamma$,

$$
\dot{\gamma}=\dot{r} \partial_{r}+\dot{v} \partial_{\vartheta}+\dot{\varphi} \partial_{\varphi}+\dot{t} \partial_{t}
$$

introduzimos as funções

$$
\begin{gathered}
\mathbb{P}(r)=g(\dot{\gamma}, V)=\left(r^{2}+a^{2}\right) E-a L_{3}, \\
\mathbb{D}(\vartheta)=\mathrm{g}(\dot{\gamma}, W)=L_{3}-a E \sin ^{2} \vartheta,
\end{gathered}
$$

observando que ao longo de uma geodésica dada (com $E$ e $L_{3}$ fixos). $\mathbb{P}$ é uma função apenas de $r$ e $\mathbb{D}$ é uma função apenas de $\vartheta$. Obtemos com estas funçòes a expansão

$$
\dot{\gamma}=\dot{i} \partial_{r}+\dot{v} \partial_{\vartheta}+\frac{\mathbb{P}}{\rho^{2} \Delta} V+\frac{\mathbb{D}}{\rho^{2} \sin ^{2} \vartheta} W
$$

o que permite substituir (3.36) por

$$
2 H=g_{\mu \nu} \dot{q}^{\mu} \dot{q}^{\nu}=\frac{\rho^{2}}{\Delta} \dot{r}^{2}+\rho^{2} \dot{\vartheta}^{2}-\frac{\mathbb{P}^{2}}{\rho^{2} \Delta}+\frac{\mathbb{D}^{2}}{\rho^{2} \sin ^{2} \vartheta},
$$

Multiplicando por $\rho^{2}=r^{2}+a^{2} \cos ^{2} v$, obtemos

$$
-\frac{\rho^{4}}{\Delta} \dot{r}^{2}+\frac{\mathbb{P}^{2}}{\lrcorner}+2 H r^{2}=\rho^{4} \dot{\vartheta}^{2}+\frac{\mathbb{D}^{2}}{\sin ^{2} \vartheta}-2 H a^{2} \cos ^{2} \vartheta
$$

Nesta equação, exceto pelo fator $\rho^{4}$, o lado esquerdo depende somente de $r$ e $\dot{r}$ enquanto que o laclo direito depende somente de $\vartheta$ e $\dot{\vartheta}$. Portanto, se $\rho$ fosse uma constante, a equação (3.42) seria separável e, em particular, ambos os lados desta equação seriam constantes.

A notável observação devida a Carter é que, embora a equação (3.42) não seja separável, ambos os lados desta equação sào constantes ao longo de cada geodésica. Em outras palavras, podemos associar a cada geodésica $\gamma$ uma constante $\mathcal{K}=\mathcal{K}_{\gamma}$, chamada a constante de Carter, dada por

$$
\Lambda:=-\frac{\rho^{4}}{\Delta} \dot{r}^{2}+\frac{\mathbb{P}^{2}}{\Delta}+2 H r^{2}
$$

ou

$$
\mathcal{K}=\rho^{4} \dot{\vartheta}^{2}+\frac{\mathbb{D}^{2}}{\sin ^{2} \vartheta}-2 H a^{2} \cos ^{2} \vartheta .
$$

Para uma demonstração direta do fato de que esta quantidade é constante ao longo de $\gamma$, veja $[12$, p. 357 . 
A definiçào do tensor de Walker-Penrose é baseada sobre uma propriedade geométrica especial do espaço-tempo de Kerr - a existência de um par de campos vetoriais $N_{+}$e $N_{-}$ tipo luz que são autoparalelos, o que significa que as curvas integrais de $N_{+}$e $N_{-}$são geodésicas tipo luz e justifica a terminologia de chamar $N_{+}$e $N_{-}$os campos vetoriais nulos principais e seus fluxos as congruências nulas principais da métrica de Kerr [12, pp. 79,95]. Explicitamente,

e então [19, pp. 321]

$$
N_{ \pm}= \pm \partial_{r}+\frac{r^{2}+a^{2}}{\Delta} \partial_{t}+\frac{a}{\Delta} \partial_{\varphi}
$$

$$
K=\frac{\Delta}{2}\left(N_{+} \odot N_{-}+N_{-} \otimes N_{+}\right)+r^{2} g .
$$

Notemos que, ao contrário do quadrado do momento angular da solução de Schwarzschild, o tensor de Walker-Penrose é indecomponível, i.e., ele não admite nenhuma representação como soma de produtos tensoriais simetrizados de campos vetoriais de Killing.

Para melhor organizar os cálculos a serem executados, é conveniente introduzir, além da base $\left(\partial_{t}, \partial_{r}, \partial_{\vartheta}, \partial_{\varphi}\right)$ de campos vetoriais coordenada, com base dual $(d t, d r, d \vartheta, d \varphi)$ de 1 formas coordenada, uma base ortonormal $\left(E_{\mu}\right)$ de campos vetoriais, com base ortonormal dual $\left(\omega^{\mu}\right)$ de 1-formas, chamadas referenciais ortonormais de Boyer-Lindquist [12, pp. 90,91]:

$$
\begin{aligned}
E_{1}=\frac{\sqrt{|\Delta|}}{\rho} \partial_{r}, & E_{3}=\frac{1}{\rho \sin \vartheta}\left(\partial_{\varphi}+a \sin ^{2} \vartheta \partial_{t}\right), \\
E_{2} & =\frac{1}{\rho} \partial_{\vartheta} \quad, \quad E_{0}=\frac{1}{\rho \sqrt{|\Delta|}}\left(\left(r^{2}+a^{2}\right) \partial_{t}+a \partial_{\varphi}\right), \\
\omega^{1} & =\frac{\rho}{\sqrt{|\Delta|}} d r \quad, \quad \omega^{3}=\frac{\sin \vartheta}{\rho}\left(\left(r^{2}+a^{2}\right) d \varphi-a d t\right), \\
\omega^{2} & =\rho d \vartheta \quad, \quad \omega^{0}=\frac{\sqrt{|\Delta|}}{\rho}\left(d t-a \sin ^{2} \vartheta d \varphi\right) .
\end{aligned}
$$

No sentido inverso,

$$
\begin{gathered}
\partial_{r}=\frac{\rho}{\sqrt{|\Delta|}} E_{1} \quad, \quad \partial_{\varphi}=\frac{\sin \vartheta}{\rho}\left(\left(r^{2}+a^{2}\right) E_{3}-a \sin \vartheta \sqrt{|\Delta|} E_{0}\right), \\
\partial_{\vartheta}=\rho E_{2} \quad, \quad \partial_{t}=\frac{1}{\rho}\left(\sqrt{|\Delta|} E_{0}-a \sin \vartheta E_{3}\right) \\
d r=\frac{\sqrt{|\Delta|}}{\rho} \omega^{1}, \quad d \varphi=\frac{1}{\rho \sin \vartheta \sqrt{|\Delta|}}\left(\sqrt{|\Delta|} \omega^{3}+a \sin \vartheta \omega^{0}\right) \\
d \vartheta=\frac{1}{\rho} \omega^{2} \quad, \quad d t=\frac{1}{\rho \sqrt{|\Delta|}}\left(a \sin \vartheta \sqrt{|\Delta|} \omega^{3}+\left(r^{2}+a^{2}\right) \omega^{0}\right) .
\end{gathered}
$$

A afirmaçào de ortonormalidade significa, mais precisamente, que os componentes do tensor métrico nesta base sào 
onde

$$
\mathrm{g}_{i j} \equiv \mathrm{g}\left(E_{i}, E_{j}\right)=\epsilon_{i} \delta_{i j}, \mathrm{~g}^{i j} \equiv \mathrm{g}\left(\omega^{i}, \omega^{j}\right)=\epsilon_{i} \delta^{i j}
$$

$$
-\epsilon_{0}=\epsilon_{1}=\epsilon, \epsilon_{2}=\epsilon_{3}=1
$$

e $\epsilon$ é o sinal da função $\Delta(+1$ nos blocos I e III, -1 no bloco II); veja (3.31). Para uso posterior, listamos também todas as derivadas covariantes dos campos vetoriais $E_{\kappa}$ ao longo dos campos vetoriais $E_{\mu}[12$, p. 95] (a demonstração encontra-se no Apêndice B):

$$
\begin{aligned}
& \nabla_{E_{1}} E_{1}=\epsilon \frac{a^{2} \sin \vartheta \cos \vartheta}{\rho^{3}} E_{2} \\
& \nabla_{E_{1}} E_{2}=-\frac{a^{2} \sin \vartheta \cos \vartheta}{\rho^{3}} E_{1} \text {, } \\
& \nabla_{E_{2}} E_{1}=\frac{r \sqrt{|\Delta|}}{\rho^{3}} E_{2} \\
& \nabla_{E_{2}} E_{2}=-\epsilon \frac{r \sqrt{|\Delta|}}{\rho^{3}} E_{1}, \\
& \nabla_{E_{0}} E_{0}=F E_{1}-\epsilon \frac{a^{2} \sin \vartheta \cos \vartheta}{\rho^{3}} E_{2}, \\
& \nabla_{E_{0}} E_{3}=\nabla_{E_{3}} E_{0}=-\epsilon \frac{a r \sin \vartheta}{\rho^{3}} E_{1}-\epsilon \frac{a \cos \vartheta \sqrt{|\Delta|}}{\rho^{3}} E_{2}, \\
& \nabla_{E_{3}} E_{3}=-\epsilon \frac{r \sqrt{|\Delta|}}{\rho^{3}} E_{1}-\frac{\left(r^{2}+a^{2}\right) \cos \vartheta}{\rho^{3} \sin \vartheta} E_{2}, \\
& \nabla_{E_{0}} E_{1}=F E_{0}+\frac{a r \sin \vartheta}{\rho^{3}} E_{3}, \\
& \nabla_{E_{0}} E_{2}=-\frac{a^{2} \sin \vartheta \cos \vartheta}{\rho^{3}} E_{0}+\epsilon \frac{a \cos \vartheta \sqrt{|\Delta|}}{\rho^{3}} E_{3}, \\
& \nabla_{E_{3}} E_{1}=-\epsilon \frac{a r \sin \vartheta}{\rho^{3}} E_{0}+\frac{r \sqrt{|\Delta|}}{\rho^{3}} E_{3}, \\
& \nabla_{E_{3}} E_{2}=-\frac{a \cos \vartheta \sqrt{|\Delta|}}{\rho^{3}} E_{0}+\frac{\left(r^{2}+a^{2}\right) \cos \vartheta}{\rho^{3} \sin \vartheta} E_{3}, \\
& \nabla_{E_{1}} E_{0}=-\frac{a r \sin \vartheta}{\rho^{3}} E_{3} \text {, } \\
& \nabla_{E_{1}} E_{3}=-\epsilon \frac{\operatorname{ar} \sin \vartheta}{\rho^{3}} E_{0}, \\
& \nabla_{E_{2}} E_{0}=\epsilon \frac{a \cos \vartheta \sqrt{|\Delta|}}{\rho^{3}} E_{3}, \\
& \nabla_{E_{2}} E_{3}=\frac{a \cos v \sqrt{|\Delta|}}{\rho^{3}} E_{0},
\end{aligned}
$$


onde usamos a abreviação

$$
F=\frac{\partial}{\partial r}\left(\frac{\sqrt{|\Delta|}}{\rho}\right)=\epsilon \frac{(r-M) \rho^{2}-r \Delta}{\rho^{3} \sqrt{|\Delta|}} .
$$

Isso posto, podemos facilmente demonstrar a seguinte

Proposição 3.1 Os campos vetoriais $N_{+}$e $N_{-}$definidos por (3.45) são campos vetoriais nulos principais da métrica de Kerr, i.e., são tipo luz e autoparalelos.

DemonstraÇÃo. Úsando (3.32) e (3.47), escrevemos

$$
N_{ \pm}= \pm \partial_{r}+\Delta^{-1} V
$$

ou ainda

$$
N_{ \pm}=\frac{\rho}{\sqrt{|\Delta|}}\left(\epsilon E_{0} \pm E_{1}\right),
$$

e introduzimos as 1-formas duais $N^{+}$e $N^{-}$, definidas por

$$
N^{ \pm}\left(E_{i}\right)=\mathrm{g}\left(N_{ \pm}, E_{i}\right)
$$

que sào

$$
N^{ \pm}=\frac{\rho}{\sqrt{|\Delta|}}\left(-\omega^{0} \pm \epsilon \omega^{1}\right) .
$$

Devido a (3.49), (3.50) e (3.70), é óbvio que $N_{+}$e $N_{-}$são campos vetoriais tipo luz. Para mostrar que sào autoparalelos, calculamos

$$
\nabla_{\epsilon E_{0} \pm E_{1}}\left(\epsilon E_{0} \pm E_{1}\right)=\nabla_{E_{0}} E_{0}+\nabla_{E_{1}} E_{1} \pm \epsilon\left(\nabla_{E_{0}} E_{1}+\nabla_{E_{1}} E_{0}\right)= \pm F\left(\epsilon E_{0} \pm E_{1}\right)
$$

e obtemos

$$
\begin{aligned}
& \nabla_{N_{ \pm}} V_{ \pm}=\frac{\rho}{\sqrt{|\Delta|}} \nabla_{\epsilon E_{0} \pm E_{1}}\left(\frac{\rho}{\sqrt{|\Delta|}}\left(\epsilon E_{0} \pm E_{1}\right)\right) \\
& =\frac{\rho}{\sqrt{|\Delta|}} \mathfrak{L}_{\epsilon E_{0} \pm E_{1}}\left(\frac{\rho}{\sqrt{|\Delta|}}\right)\left(\epsilon E_{0} \pm E_{1}\right)+\frac{\rho^{2}}{|\Delta|} \nabla_{\epsilon E_{0} \pm E_{1}}\left(\epsilon E_{0} \pm E_{1}\right) \\
& =\left( \pm \frac{\partial}{\partial r}+\frac{\left(r^{2}+a^{2}\right)}{\Delta} \frac{\partial}{\partial t}+\frac{a}{\Delta} \frac{\partial}{\partial \varphi}\right)\left(\frac{\rho}{\sqrt{|\Delta|}}\right)\left(\epsilon E_{0} \pm E_{1}\right) \\
& \pm \frac{\rho^{2}}{|\Delta|} \frac{\partial}{\partial r}\left(\frac{\sqrt{|\Delta|}}{\rho}\right)\left(\epsilon E_{0} \pm E_{1}\right) \\
& =0 \text {. }
\end{aligned}
$$


Passamos a calcular os componentes do tensor de Walker-Penrose relativos ao referencial ortonormal de Boyer-Lindquist:

$$
\begin{aligned}
& \left(K^{i j}\right)=\left(\begin{array}{cccc}
\Delta\left(N_{+}^{0} N_{-}^{-0}\right)+r^{2} \mathrm{~g}^{00} & \frac{1}{2} \Delta\left(N_{+}^{0} N_{-}^{1}+N_{+}^{1} N_{-}^{0}\right) & 0 & 0 \\
\frac{1}{2} \Delta\left(N_{+}^{1} N_{-}^{0}+N_{+}^{0} N_{-}^{1}\right) & \Delta\left(N_{+}^{1} N_{-}^{1}\right)+r^{2} \mathrm{~g}^{11} & 0 & 0 \\
0 & 0 & r^{2} \mathrm{~g}^{22} & 0 \\
0 & 0 & 0 & r^{2} g^{33}
\end{array}\right) \\
& =\left(\begin{array}{cccc}
\epsilon \rho^{2}+\epsilon_{0} r^{2} & 0 & 0 & 0 \\
0 & -\epsilon \rho^{2}+\epsilon_{1} r^{2} & 0 & 0 \\
0 & 0 & \epsilon_{2} r^{2} & 0 \\
0 & 0 & 0 & \epsilon_{3} r^{2}
\end{array}\right) \\
& \left(K_{i j}\right)=\left(\begin{array}{cccc}
\Delta\left(N_{0}^{+} N_{0}^{-}\right)+r^{2} \mathrm{~g}_{00} & \frac{1}{2} \Delta\left(N_{0}^{+} N_{1}^{-}+N_{1}^{+} N_{0}^{-}\right) & 0 & 0 \\
\frac{1}{2} \Delta\left(N_{1}^{+} x_{0}^{-}+N_{0}^{+} N_{1}^{-}\right) & \Delta\left(N_{1}^{+} N_{1}^{-}\right)+r^{2} \mathrm{~g}_{11} & 0 & 0 \\
0 & 0 & r^{2} \mathrm{~g}_{22} & 0 \\
0 & 0 & 0 & r^{2} \mathrm{~g}_{33}
\end{array}\right) \\
& =\left(\begin{array}{cccc}
\epsilon \rho^{2}+\epsilon_{0} r^{2} & 0 & 0 & 0 \\
0 & -\epsilon \rho^{2}+\epsilon_{1} r^{2} & 0 & 0 \\
0 & 0 & \epsilon_{2} r^{2} & 0 \\
0 & 0 & 0 & \epsilon_{3} r^{2}
\end{array}\right)
\end{aligned}
$$

com o resultado

$$
\left(K^{i j}\right)=\left(K_{i j}\right)=\left(\begin{array}{cccc}
\epsilon a^{2} \cos ^{2} \vartheta & 0 & 0 & 0 \\
0 & -\epsilon a^{2} \cos ^{2} \vartheta & 0 & 0 \\
0 & 0 & r^{2} & 0 \\
0 & 0 & 0 & r^{2}
\end{array}\right)
$$

Também é útil conhecer os componentes relativos às coordenadas de Boyer-Lindquist. Os componentes contravariantes sào

$$
\begin{aligned}
K^{\prime t t}=K(d t, d t) & =\frac{1}{\rho^{2}|\Delta|}\left(a^{2} \sin ^{2} \vartheta|\Delta| K^{33}+\left(r^{2}+a^{2}\right)^{2} K^{\cdot 00}\right) \\
& =\frac{a^{2}}{\rho^{2} \Delta}\left(r^{2} \sin ^{2} \vartheta \Delta+\left(r^{2}+a^{2}\right)^{2} \cos ^{2} \vartheta\right) \\
& =\frac{a^{2}}{\rho^{2} \Delta}\left(r^{2}\left(r^{2}+a^{2}\right) \sin ^{2} \vartheta+\left(r^{2}+a^{2}\right)^{2} \cos ^{2} \vartheta-2 m r^{3} \sin ^{2} \vartheta\right) \\
& =\frac{a^{2}}{\Delta}\left(r^{2}+a^{2}-\frac{2 m r^{3} \sin ^{2} \vartheta}{\rho^{2}}\right)
\end{aligned}
$$




$$
\begin{aligned}
& K^{r r}=K(d r, d r)=\frac{|\Delta|}{\rho^{2}} K^{11}=-\frac{a^{2} \Delta}{\rho^{2}} \cos ^{2} \vartheta \\
& K^{\vartheta \vartheta}=K(d \vartheta, d \vartheta)=\frac{1}{\rho^{2}} K^{22}=\frac{r^{2}}{\rho^{2}} \\
& K^{\varphi \varphi}=K(d \varphi, d \varphi)=\frac{1}{\rho^{2} \sin ^{2} \vartheta|\Delta|}\left(|\Delta| K^{\cdot 33}+a^{2} \sin ^{2} \vartheta K^{\circ 00}\right) \\
& =\frac{1}{\rho^{2} \sin ^{2} \vartheta \Delta}\left(r^{2} \Delta+a^{4} \sin ^{2} \vartheta \cos ^{2} \vartheta\right) \\
& =\frac{1}{\rho^{2} \sin ^{2} \vartheta \Delta}\left(r^{2}\left(r^{2}+a^{2}\right)+a^{4} \sin ^{2} \vartheta \cos ^{2} \vartheta-2 m r^{3}\right) \\
& =\frac{1}{\rho^{2} \sin ^{2} \vartheta \Delta}\left(\left(r^{2}+a^{2} \cos ^{2} \vartheta\right)\left(r^{2}+a^{2} \sin ^{2} \vartheta\right)-2 m r^{3}\right) \\
& =\frac{1}{\sin ^{2} \vartheta \Delta}\left(r^{2}+a^{2} \sin ^{2} \vartheta-\frac{2 m r^{3}}{\rho^{2}}\right) \\
& K^{t \varphi}=K(d t, d \varphi)=\frac{a}{\rho^{2}|\Delta|}\left(|\Delta| K^{-33}+\left(r^{2}+a^{2}\right) K^{-00}\right) \\
& =\frac{a}{\rho^{2} \Delta}\left(r^{2} \Delta+a^{2}\left(r^{2}+a^{2}\right) \cos ^{2} \vartheta\right) \\
& =\frac{a}{\rho^{2} \Delta}\left(r^{2}\left(r^{2}+a^{2}\right)+a^{2}\left(r^{2}+a^{2}\right) \cos ^{2} \vartheta-2 m r^{3}\right) \\
& =\frac{a}{\Delta}\left(r^{2}+a^{2}-\frac{2 m r^{3}}{\rho^{2}}\right) \sin ^{2} \vartheta
\end{aligned}
$$

e os componentes covariantes sào

$$
\begin{aligned}
K_{t t}=K\left(\partial_{t}, \partial_{t}\right) & =\frac{1}{\rho^{2}}\left(|\Delta| K_{00}+a^{2} \sin ^{2} \vartheta K_{33}\right)=\frac{a^{2}}{\rho^{2}}\left(\Delta \cos ^{2} \vartheta+r^{2} \sin ^{2} \vartheta\right) \\
& =\frac{a^{2}}{\rho^{2}}\left(\left(r^{2}+a^{2}\right) \cos ^{2} \vartheta+r^{2} \sin ^{2} \vartheta-2 m r \cos ^{2} \vartheta\right) \\
& =a^{2}\left(1-\frac{2 m r \cos ^{2} \vartheta}{\rho^{2}}\right) \\
K_{r r}=K\left(\partial_{r}, \partial_{r}\right) & =\frac{\rho^{2}}{|\Delta|} K_{11}=-\frac{a^{2} \rho^{2}}{\Delta} \cos ^{2} \vartheta \\
K_{\vartheta \vartheta}=K\left(\partial_{\vartheta}, \partial_{\vartheta}\right) & =\rho^{2} K_{22}=\rho^{2} r^{2}
\end{aligned}
$$




$$
\begin{aligned}
& \kappa_{\varphi \varphi}=K^{\prime}\left(\partial_{\varphi}, \partial_{\varphi}\right)=\frac{\sin ^{2} \vartheta}{\rho^{2}}\left(\left(r^{2}+a^{2}\right)^{2} K_{33}+a^{2} \sin ^{2} \vartheta|\Delta| K_{00}\right) \\
& =\frac{\sin ^{2} \vartheta}{\rho^{2}}\left(r^{2}\left(r^{2}+a^{2}\right)^{2}+a^{4} \sin ^{2} \vartheta \cos ^{2} \vartheta \Delta\right) \\
& =\frac{\sin ^{2} \vartheta}{\rho^{2}}\left(r^{2}\left(r^{2}+a^{2}\right)^{2}+a^{4} \sin ^{2} \vartheta \cos ^{2} \vartheta\left(r^{2}+a^{2}\right)\right. \\
& \left.-2 m a^{4} r \sin ^{2} \vartheta \cos ^{2} \vartheta\right) \\
& =\frac{\sin ^{2} \vartheta}{\rho^{2}}\left(\left(r^{2}+a^{2}\right)\left(r^{2}+a^{2} \cos ^{2} \vartheta\right)\left(r^{2}+a^{2} \sin ^{2} \vartheta\right)\right. \\
& \left.-2 m a^{4} r \sin ^{2} \vartheta \cos ^{2} \vartheta\right) \\
& =\left(\left(r^{2}+a^{2}\right)\left(r^{2}+a^{2} \sin ^{2} \vartheta\right)-\frac{2 m a^{4} r \sin ^{2} \vartheta \cos ^{2} \vartheta}{\rho^{2}}\right) \sin ^{2} \vartheta \\
& K_{t \varphi}=K\left(\partial_{t}, \partial_{\varphi}\right)=-\frac{\sin \vartheta}{\rho^{2}}\left(|\Delta| a \sin \vartheta K_{00}+a\left(r^{2}+a^{2}\right) \sin \vartheta \kappa_{33}\right) \\
& =-\frac{a \sin ^{2} \vartheta}{\rho^{2}}\left(a^{2} \Delta \cos ^{2} \vartheta+r^{2}\left(r^{2}+a^{2}\right)\right) \\
& =-\frac{a \sin ^{2} \vartheta}{\rho^{2}}\left(a^{2}\left(r^{2}+a^{2}\right) \cos ^{2} \vartheta+r^{2}\left(r^{2}+a^{2}\right)-2 m a^{2} r \cos ^{2} \vartheta\right) \\
& =-a\left(r^{2}+a^{2}-\frac{2 m a^{2} r \cos ^{2} \vartheta}{\rho^{2}}\right) \sin ^{2} \vartheta
\end{aligned}
$$

Temos, portanto, para o tensor contravariante

$$
\begin{aligned}
K= & \frac{a^{2}}{\Delta}\left(r^{2}+a^{2}-\frac{2 m r^{3} \sin ^{2} \vartheta}{\rho^{2}}\right) \partial_{t}^{2}-\frac{a^{2} \Delta}{\rho^{2}} \cos ^{2} \vartheta \partial_{r}^{2}+\frac{r^{2}}{\rho^{2}} \partial_{\vartheta}^{2} \\
& +\frac{1}{\sin ^{2} \vartheta \Delta}\left(r^{2}+a^{2} \sin ^{2} \vartheta-\frac{2 m r^{3}}{\rho^{2}}\right) \partial_{\varphi}^{2} \\
& +\frac{a}{\Delta}\left(r^{2}+a^{2}-\frac{2 m r^{3}}{\rho^{2}}\right) \sin ^{2} \vartheta 2 \partial_{t} \partial_{\varphi},
\end{aligned}
$$

e para o tensor covariante

$$
\begin{aligned}
K= & a^{2}\left(1-\frac{2 m r \cos ^{2} \vartheta}{\rho^{2}}\right) d t^{2}-\frac{a^{2} \rho^{2}}{\Delta} \cos ^{2} \vartheta d r^{2}+\rho^{2} r^{2} d \vartheta^{2} \\
& +\left(\left(r^{2}+a^{2}\right)\left(r^{2}+a^{2} \sin ^{2} \vartheta\right)-\frac{2 m a^{4} r \sin ^{2} \vartheta \cos ^{2} \vartheta}{\rho^{2}}\right) \sin ^{2} \vartheta d \varphi^{2} \\
& -a\left(r^{2}+a^{2}-\frac{2 m a^{2} r \cos ^{2} \vartheta}{\rho^{2}}\right) \sin ^{2} \vartheta 2 d t d \varphi
\end{aligned}
$$


A demonstração do fato de que o colchete de Schouten de $K$ com o tensor métrico se anula requer um cálculo explícito do tensor $S$ com componentes covariantes

$$
S_{k i j}=\nabla_{E_{k}} K_{i j}+\nabla_{E_{i}} K_{j k}+\nabla_{E_{j}} K_{k i} .
$$

Com as ferramentas desenvolvidas, podemos mostrar que todos se anulam (os detalhes encontram-se no Apêndice C) e assim concluir:

Teorema 3.1 O tensor de Walker-Penrose é um campo tensorial de Killing para a métrica de lierr.

A quantidade conservada para o fluxo geodésico associada ao tensor de Walker-Penrose. em termos de coordenadas em $T Q$, é

$$
\begin{aligned}
\mathcal{K}= & a^{2}\left(1-\frac{2 m r \cos ^{2} \vartheta}{\rho^{2}}\right) \dot{t}^{2}-\frac{a^{2} \rho^{2}}{\Delta} \cos ^{2} \vartheta \dot{r}^{2}+\rho^{2} r^{2} \dot{\vartheta}^{2} \\
& +\left(\left(r^{2}+a^{2}\right)\left(r^{2}+a^{2} \sin ^{2} \vartheta\right)-\frac{2 m a^{4} r \sin ^{2} \vartheta \cos ^{2} \vartheta}{\rho^{2}}\right) \sin ^{2} \vartheta \dot{\varphi}^{2} \\
& -a\left(r^{2}+a^{2}-\frac{2 m a^{2} r \cos ^{2} \vartheta}{\rho^{2}}\right) \sin ^{2} \vartheta 2 \dot{t} \dot{\varphi}
\end{aligned}
$$

Queremos mostrar que esta constante coincide com a constante de Carter introduzida anteriormente. Para tanto, calculamos primeiro o produto escalar dos vetores nulos $N_{ \pm}$, dados pela equação (3.69), com o vetor tangente à geodésica $\gamma$, dado pela equação (3.40):

$$
\mathrm{g}\left(N_{ \pm}, \dot{\gamma}\right)= \pm \dot{r} \mathrm{~g}_{r r}+\frac{\mathbb{P}}{\rho^{2} \Delta^{2}} g(V, V)=\frac{1}{\Delta}\left( \pm \rho^{2} \dot{r}-\mathbb{P}\right) .
$$

Considerando agora a equação (3.46) do tensor de Killing teremos que

$$
\begin{aligned}
K(\dot{\gamma}, \dot{\gamma}) & =\Delta \mathrm{g}\left(N_{+}, \dot{\gamma}\right) \mathrm{g}\left(N_{-}, \dot{\gamma}\right)+r^{2} \mathrm{~g}(\dot{\gamma}, \dot{\gamma}) \\
& =\frac{1}{\Delta}\left(\rho^{2} \dot{r}-\mathbb{P}\right)\left(-\rho^{2} \dot{r}-\mathbb{P}\right)+2 H r^{2} \\
& =\frac{1}{\Delta}\left(\mathbb{P}^{2}-\rho^{4} \dot{r}^{2}\right)+2 H r^{2} \\
& =\mathcal{K},
\end{aligned}
$$

de acordo com a equação (3.43). Observemos ainda que a constante de Carter realmente é uma perturbação da quantidade conservada do fluxo geodésico na solução de Schwarzschild que é quadrática nos momentos, o $L^{2}$, pois

$$
\left.\mathcal{K}\right|_{a=0}=r^{4} \dot{v}^{2}+r^{4} \sin ^{2} \vartheta \dot{\varphi}^{2}=L^{2} .
$$


Para demonstrar que as quatro quantidades conservadas $E, L_{3}, \mathcal{K}$ e $H$ do fluxo geodésico na solução de Kerr estão em involução, consideremos os anti-homomorfismos de álgebras de Lie (2.21) e (2.29). Pela própria definição dos campos vetoriais e tensoriais de Killing, precisamos calcular somente os colchetes de Poisson da constante de Carter $\mathcal{K}$ com a energia $E$ e com o momento angular $L_{3}$, uma vez que devido ao Teorema 3.1, o colchete de Poisson

$$
\{2 H \cdot \mathcal{\Lambda}\}=\left\{\mathcal{C}(\mathrm{g}), \mathcal{C}\left(K^{\prime}\right)\right\}=-\mathcal{C}\left(\left[\mathrm{g}, K^{\prime}\right]\right)
$$

se anula. Observando as expressões do tensor de Killing (3.7.5) e (3.76), vemos que os componentes de $K$ nas coordenadas de Boyer-Lindquist não dependem de $t$ ou de $\varphi$ e portanto os colchetes de Poisson

$$
\begin{aligned}
\left\{E \cdot \Lambda^{\prime}\right\} & =\left\{\mathcal{C}\left(\partial_{t}\right), \mathcal{C}\left(K^{\prime}\right)\right\}=-\mathcal{C}\left(\left[\partial_{t}, K^{\prime}\right]\right) \\
\left\{L_{3} \cdot \Lambda^{\prime}\right\} & =\left\{\mathcal{C}\left(\partial_{\varphi}\right), \mathcal{C}\left(K^{\prime}\right)\right\}=-\mathcal{C}\left(\left[\partial_{\varphi} \cdot K^{\prime}\right]\right)
\end{aligned}
$$

também se anulam.

Mostremos finalmente que as quantidades conservadas $E, L_{3}, \mathcal{K}$ e $H$ são funcionalmente independentes, como pode ser verificado através de um cálculo explícito do posto da matriz Jacobiana

$$
\begin{aligned}
& \frac{\partial\left(E, L_{3}, \mathcal{K}, 2 H\right)}{\partial(t, r, \vartheta, \varphi, \dot{t}, \dot{r}, \dot{\vartheta}, \dot{\varphi})}=\left(\begin{array}{cccccccc}
\partial_{t} E & \partial_{r} E & \partial_{\vartheta} E & \partial_{\varphi} E & \partial_{i} E & \partial_{\dot{r}} E & \partial_{\dot{\vartheta}} E & \partial_{\dot{\varphi}} E \\
\partial_{t} L_{3} & \partial_{r} L_{3} & \partial_{\vartheta} L_{3} & \partial_{\varphi} L_{3} & \partial_{\dot{t}} L_{3} & \partial_{\dot{r}} L_{3} & \partial_{\dot{\vartheta}} L_{3} & \partial_{\dot{\varphi}} L_{3} \\
\partial_{t} \mathcal{K} & \partial_{r} \mathcal{K} & \partial_{\vartheta} \mathcal{K} & \partial_{\varphi} \mathcal{K} & \partial_{\dot{t}} \mathcal{K} & \partial_{i} \mathcal{K} & \partial_{\dot{\vartheta}} \mathcal{K} & \partial_{\dot{\varphi}} \mathcal{K} \\
2 \dot{\partial}_{t} H & 2 \partial_{r} H & 2 \partial_{\vartheta} H & 2 \partial_{\varphi} H & 2 \partial_{\dot{t}} H & 2 \partial_{\dot{r}} H & 2 \partial_{\dot{\vartheta}} H & 2 \dot{\partial}_{\dot{\varphi}} H
\end{array}\right) \\
& =\left(\begin{array}{cccc}
0 & \partial_{r} E & \partial_{\vartheta} E & 0 \\
0 & \partial_{r} L_{3} & \partial_{\vartheta} L_{3} & 0 \\
0 & \partial_{r} \mathcal{K} & \partial_{\vartheta} \mathcal{K} & 0 \\
0 & 2 \partial_{r} H & 2 \partial_{\vartheta} H & 0
\end{array}\right. \\
& \left.\begin{array}{cccc}
-\mathrm{g}_{t t} & 0 & 0 & -\mathrm{g}_{t \varphi} \\
\mathrm{g}_{t_{\varphi}} & 0 & 0 & \mathrm{~g}_{\varphi \varphi} \\
\partial_{\dot{t}} \mathcal{\Lambda} & -\frac{2 a^{2} \rho^{2} \cos ^{2} \vartheta}{\Delta} \dot{r} & 2 r^{2} \mathrm{~g}_{\vartheta \vartheta} \dot{\vartheta} & \partial_{\dot{\phi}} \mathcal{K} \\
2 \dot{\partial}_{\dot{i}} H & 2 \mathrm{~g}_{r r} \dot{r} & 2 \mathrm{~g}_{\vartheta \vartheta} \dot{\vartheta} & 2 \partial_{\dot{\varphi}} H
\end{array}\right) .
\end{aligned}
$$

O subdeterminante da derivada em relação às velocidades é

$$
\frac{\partial\left(E, L_{3}, \mathcal{K}, 2 H\right)}{\partial(\dot{t}, \dot{r}, \dot{\nu} \cdot \dot{\varphi})}=\operatorname{det}\left(\begin{array}{cccc}
-\mathrm{g}_{t t} & 0 & 0 & -\mathrm{g}_{t \varphi} \\
\mathrm{g}_{t \varphi} & 0 & 0 & \mathrm{~g}_{\varphi \varphi} \\
\partial_{\dot{t}} \mathcal{K} & -\frac{2 a^{2} \rho^{2} \cos ^{2} \vartheta}{\Delta} \dot{r} & 2 r^{2} \mathrm{~g}_{\partial \vartheta} \dot{\nu} & \partial_{\dot{\varphi}} \mathcal{K} \\
2 \partial_{\dot{t}} H & 2 \mathrm{~g}_{r r} \dot{r} & 2 \mathrm{~g}_{\partial \vartheta} \dot{j} & 2 \partial_{\dot{\varphi}} H
\end{array}\right)
$$




$$
\begin{aligned}
& =-\mathrm{g}_{t t} \operatorname{det}\left(\begin{array}{ccc}
0 & 0 & \mathrm{~g}_{\varphi \varphi} \\
-\frac{2 a^{2} \rho^{2} \cos ^{2} \vartheta}{\Delta} \dot{r} & 2 r^{2} \mathrm{~g}_{\vartheta \vartheta} \dot{\vartheta} & \partial_{\dot{\vartheta}} \mathcal{K} \\
2 \mathrm{~g}_{r r} \dot{r} & 2 \mathrm{~g}_{\vartheta \vartheta} \dot{\varphi} & 2 \partial_{\dot{\varphi}} H
\end{array}\right) \\
& +\mathrm{g}_{t \varphi} \operatorname{det}\left(\begin{array}{ccc}
\mathrm{g}_{t \varphi} & 0 & 0 \\
\partial_{\dot{t}} \mathcal{K} & -\frac{2 a^{2} \rho^{2} \cos ^{2} \vartheta}{\Delta} \dot{r} & 2 r^{2} \mathrm{~g}_{\vartheta \vartheta} \dot{\vartheta} \\
2 \partial_{i} H & 2 \mathrm{~g}_{r r} \dot{r} & 2 \mathrm{~g}_{\vartheta \vartheta} \dot{\vartheta}
\end{array}\right) \\
& =-\left(\mathrm{g}_{t t} \mathrm{~g}_{\varphi \varphi}-\mathrm{g}_{t \varphi}^{2}\right) \operatorname{det}\left(\begin{array}{cc}
-\frac{2 a^{2} \rho^{2} \cos ^{2} \vartheta}{\Delta} \dot{r} & 2 r^{2} \mathrm{~g}_{\vartheta \vartheta} \dot{\vartheta} \\
2 \mathrm{~g}_{r r} \dot{r} & 2 \mathrm{~g}_{\vartheta \vartheta} \dot{\vartheta}
\end{array}\right) \\
& =\left[\left(1-\frac{2 m r}{\rho^{2}}\right)\left(r^{2}+a^{2}+\frac{2 m a^{2} r \sin ^{2} \vartheta}{\rho^{2}}\right) \sin ^{2} \vartheta+\frac{4 m^{2} a^{2} r^{2} \sin ^{4} \vartheta}{\rho^{4}}\right] \\
& \times 4 \rho^{2} \dot{r} \dot{\vartheta}\left(-\frac{a^{2} \rho^{2} \cos ^{2} \vartheta}{\Delta}-\frac{\rho^{2} r^{2}}{\Delta}\right) \\
& =\left[\left(r^{2}+a^{2}+\frac{2 m a^{2} r \sin ^{2} \vartheta}{\rho^{2}}-\frac{2 m r}{\rho^{2}} \rho^{2}-\frac{2 m r}{\rho^{2}} a^{2} \sin ^{2} \vartheta-\frac{4 m^{2} a^{2} r^{2} \sin ^{2} \vartheta}{\rho^{4}}\right) \sin ^{2} \vartheta\right. \\
& \left.+\frac{4 m^{2} a^{2} r^{2} \sin ^{4} \vartheta}{\rho^{4}}\right] 4 \rho^{4} \frac{r^{2}+a^{2} \cos ^{2} \vartheta}{\Delta} \dot{r} \dot{\jmath} \\
& =-4 \rho^{6} \sin ^{2} \vartheta \dot{r} \dot{v} .
\end{aligned}
$$

Definindo

$$
Z=Z_{\sin \vartheta} \cup Z_{\dot{\vartheta}} \cup Z_{\dot{r}}
$$

onde

$$
\begin{aligned}
Z_{\sin \vartheta} & =\{(q, \dot{q}) / \sin \vartheta=0\} \\
Z_{\dot{\vartheta}} & =\{(q, \dot{q}) / \dot{\vartheta}=0\}, \\
Z_{\dot{r}} & =\{(q, \dot{q}) / \dot{r}=0\} .
\end{aligned}
$$

isso mostra que os gradientes das quantidades conservadas são linearmente independentes fora de $Z$, e como $Z$ é um subconjunto de medida nula no espaço de fase $T Q$, concluímos que as quantidades conservadas são funcionalmente independentes. (Na verdade, calculando outros subdeterminantes, podemos mostrar que o subconjunto de dependência funcional é ainda menor do que o $Z$ dado aqui, mas nào precisaremos deste fato.) Resumindo, podemos concluir que o fluxo geodésico na solução de Kerr é um sistema Hamiltoniano completamente integrável. 


\section{Apêndice A}

\section{Colchete de Schouten}

Seja $M$ uma variedade. Definimos um campo multivetorial simétrico de grau $p$ sobre $M$ como sendo um campo tensorial de grau $p$ totalmente simétrico e contravariante, ou seja, uma seção do fibrado vetorial $\bigvee^{p} T M$, e um campo multivetorial simétrico sobre $M$, sem especificação do grau. como sendo uma combinação linear de campos multivetoriais simétricos sobre $M$ de vários graus, ou seja, uma seção do fibrado vetorial

$$
\bigvee T M=\bigoplus_{p=0}^{\infty} V^{p} T M
$$

O espaço dos campos multivetoriais simétricos de grau $p$ sobre $M$ será denotado por

$$
\mathfrak{X}^{p}(M)=\Gamma\left(\bigvee^{p} T M\right)
$$

e o espaço dos campos multivetoriais simétricos sobre $M$ por

$$
\mathfrak{X}^{\vee}(M)=\Gamma(\bigvee T M) .
$$

Em coordenadas locais $x^{i}$, um campo multivetorial simétrico $P$ de grau $p$ possue uma representaçào da forma

$$
P=\frac{1}{p !} P^{i_{1} \ldots i_{p}} \partial_{i_{1}} \vee \ldots \vee \partial_{i_{p}}
$$

onde $\vee$ representa o produto tensorial simetrizado, i.e., temos para campos vetoriais $X_{1}, \ldots X_{p} \in \mathfrak{X}(M)$,

$$
X_{1} \vee \ldots \vee X_{p}=\frac{1}{p !} \sum_{\sigma \in S_{p}} X_{\sigma(1)} \odot \ldots \oslash X_{\sigma(p)}
$$

Em componentes, a operação de simetrizaçào será indicada colocando os índices correspondentes entre parênteses:

$$
P^{\left(i_{1} \ldots i_{p}\right)}=\frac{1}{p !} \sum_{\sigma \in S_{p}} P^{i_{\sigma(1) \ldots i}(p)}
$$


Em particular. para $p=2$,

$$
P^{(i j)}=\frac{1}{2}\left(P^{i j}+P^{j i}\right) .
$$

Em 1940, Schouten introduziu um colchete para campos multivetoriais, hoje chamado o colchete de Schouten, que pode ser visto como uma família de aplicaçòes

$$
[., .]: \Gamma\left(\bigvee^{p} T M\right) \times \Gamma\left(\bigvee^{q} T M\right) \longrightarrow \Gamma\left(\bigvee^{p+q-1} T M\right),
$$

com as seguintes propriedades:

(i) Bilinearidade (sobre $\mathbb{R})$ : Para $\alpha_{1}, \alpha_{2} \in \mathbb{R}, P, P_{1}, P_{2} \in \Gamma\left(\bigvee^{p} T M\right)$ e $Q, Q_{1}, Q_{2} \in$ $\Gamma\left(\bigvee^{q} T M\right)$,

$$
\begin{aligned}
& {\left[\alpha_{1} P_{1}+\alpha_{2} P_{2}, Q\right]=\alpha_{1}\left[P_{1}, Q\right]+\alpha_{2}\left[P_{2}, Q\right]} \\
& {\left[P, \alpha_{1} Q_{1}+\alpha_{2} Q_{2}\right]=\alpha_{1}\left[P, Q_{1}\right]+\alpha_{2}\left[P, Q_{2}\right] .}
\end{aligned}
$$

(ii) Antisimetria: Para $P \in \Gamma\left(\bigvee^{p} T M\right), Q \in \Gamma\left(\bigvee^{q} T M\right)$,

$$
[P, Q]+[Q, P]=0 .
$$

(iii) Identidade de Jacobi : Para $P \in \Gamma\left(\bigvee^{p} T M\right), Q \in \Gamma\left(\bigvee^{q} T M\right), R \in \Gamma\left(\bigvee^{r} T M\right)$

$$
[P,[Q, R]]+[Q,[R, P]]+[R,[P, Q]]=0 .
$$

(iv) Regra de Leibniz com respeito ao produto simétrico: Para $P \in \Gamma\left(\bigvee^{p} T M\right), Q \in$ $\Gamma\left(\bigvee^{q} T M\right), R \in \Gamma\left(\bigvee^{r} T M\right)$,

$$
[P, Q \vee R]=[P, Q] \vee R+Q \vee[P, R] .
$$

(v) Normalizaçào: O colchete de Schouten de duas funções vale 0 , o colchete de Schouten de um campo vetorial $X$ e uma função $f$ é igual à derivada de $f$ ao longo de $X$ e o colchete de Schouten de dois campos vetoriais é igual ao seu colchete de Lie.

Obviamente, a condição de normalização e uma parte da regra de Leibniz podem ser combinadas na afirmaçào de que o colchete de Schouten entre um campo vetorial $X$ e um campo multivetorial $P$ qualquer é igual à derivada de Lie de $P$ ao longo de $X$ :

$$
[X, P]=\mathfrak{L}_{X} P=-[P, X] .
$$

Também é fácil ver que para campos multivetoriais decomponíveis, devemos ter

$$
\begin{aligned}
& {\left[X_{1} \vee \ldots \vee X_{p}, Y_{1} \vee \ldots \vee Y_{q}\right]} \\
& \quad=\sum_{i=1}^{p} \sum_{j=1}^{q}\left[X_{i}, Y_{j}\right] \vee X_{1} \vee \ldots \vee \hat{X}_{i} \vee \ldots \vee X_{p} \vee Y_{1} \vee \ldots \vee \hat{Y}_{j} \vee \ldots \vee Y_{q},
\end{aligned}
$$


onde o símbolo. significa que o argumento correspondente deve ser omitido. Em coordenadas locais $x^{i}$, escrevendo

$$
P=\frac{1}{p !} P^{i_{1} \ldots i_{p}} \partial_{i_{1}} \vee \ldots \vee \partial_{i_{p}} \quad, \quad Q=\frac{1}{q !} Q^{j_{1} \ldots j_{q}} \partial_{j_{1}} \vee \ldots \vee \partial_{j_{q}}
$$

e

$$
[P . Q]=\frac{1}{(p+q-1) !}[P, Q]^{k_{1} \ldots k_{p+q-1}} \partial_{k_{1}} \vee \ldots \vee \partial_{k_{p+q-1}}
$$

obtemos

$$
\begin{aligned}
{[P, Q]^{k_{1} \ldots k_{p+q-1}}=} & \frac{1}{(p-1) ! q !} \delta_{i_{1} \ldots i_{p-1} j_{1} \ldots j_{q}}^{k_{1} \ldots k_{p+q-1}} P^{l i_{1} \ldots i_{p-1}} \partial_{l} Q^{j_{1} \ldots j_{q}} \\
& -\frac{1}{p !(q-1) !} \delta_{i_{1} \ldots i_{p} j_{2} \ldots j_{q}}^{k_{1} \ldots k_{p+q-1}} Q^{l j_{2} \ldots j_{q}} \partial_{l} P^{i_{1} \ldots i_{p}}
\end{aligned}
$$

onde $\delta_{l_{1} \ldots l_{r}}^{k_{1} \ldots k_{r}}$ significa o símbolo de Kronecker totalmente simetrizado, valendo 1 se $\left(k_{1}, \ldots, k_{r}\right)$ e $\left(l_{1}, \ldots, l_{r}\right)$ são relacionados por uma permutação (par ou impar) e 0 caso contrário. Em termos da notação para a simetrização introduzida em (A.3), esta equação pode ser reescrita como

$$
\begin{aligned}
{[P, Q]^{k_{1} \ldots k_{p+q-1}}=} & \frac{(p+q-1) !}{(p-1) ! q !} P^{l\left(k_{1} \ldots k_{p-1}\right.} \partial_{l} Q^{\left.k_{p} \ldots k_{p+q-1}\right)} \\
& -\frac{(p+q-1) !}{p !(q-1) !} Q^{l\left(k_{1} \ldots k_{q-1}\right.} \partial_{l} P^{\left.k_{q} \ldots k_{p+q-1}\right)} .
\end{aligned}
$$

Reciprocamente, essas fórmulas podem ser usadas para demonstrar a existência do colchete de Schouten. Tal procedimento é análogo à maneira como se demonstra a existência da derivada exterior $d$ para formas diferenciais. Outra propriedade que o colchete de Schouten tem em comum com a derivada exterior é o fato de que na equaçào (A.14), podemos substituir as derivadas comuns $\partial_{l}$ por derivadas covariantes $\nabla_{l}$ com respeito a qualquer conexão $\nabla$ em $M$ sem torção:

$$
\begin{aligned}
{[P, Q]^{k_{1} \ldots k_{p+q-1}}=} & \frac{(p+q-1) !}{(p-1) ! q !} P^{l\left(k_{1} \ldots k_{p-1}\right.} \nabla_{l} Q^{\left.k_{p} \ldots k_{p+q-1}\right)} \\
& -\frac{(p+q-1) !}{p !(q-1) !} Q^{l\left(k_{1} \ldots k_{q-1}\right.} \nabla_{l} P^{\left.k_{q} \ldots k_{p+q-1}\right)}
\end{aligned}
$$

De fato, a diferença entre o lado direito de (A.15) e o lado direito de (A.14) é

$$
\begin{aligned}
=\frac{(p+q-1) !}{p ! q !} & \left(\sum_{j=p}^{p+q-1} p P^{l\left(k_{1} \ldots k_{p-1}\right.} \Gamma_{l m}^{k_{j}} Q^{\left.k_{p} \ldots k_{j-1} m k_{j+1} \ldots k_{p+q-1}\right)}\right. \\
& \left.-\sum_{i=q}^{p+q-1} q Q^{l\left(k_{1} \ldots k_{q-1}\right.} \Gamma_{l m}^{k_{i}} P^{\left.k_{q} \ldots k_{i-1} m k_{i+1} \ldots k_{p+q-1}\right)}\right)
\end{aligned}
$$




$$
\begin{gathered}
=\frac{(p+q-1) !}{p ! q !}\left(p q P^{l\left(k_{1} \ldots k_{p-1}\right.} \Gamma_{l m}^{k_{p}} Q^{\left.k_{p+1} \ldots k_{p+q-1} m\right)}\right. \\
\left.-q p Q^{l\left(k_{1} \ldots k_{q-1}\right.} \Gamma_{l m}^{k_{q}} P^{\left.k_{q+1} \ldots k_{p+q-1} m\right)}\right) \\
=\frac{(p+q-1) !}{p ! q !}\left(p q P^{\left(l k_{1} \ldots k_{p-1}\right.} \Gamma_{l m}^{k_{p}} Q^{\left.k_{p+1} \ldots k_{p+q-1} m\right)}\right. \\
\left.-q p Q^{\left(l k_{1} \ldots k_{q-1}\right.} \Gamma_{l m}^{k_{q}} P^{\left.k_{q+1} \ldots k_{p+q-1} m\right)}\right),
\end{gathered}
$$

o que se anula devido à simetria dos símbolos de Christoffel $\Gamma_{l m}^{k}$ em $l$ e $m$.

Finalmente, mencionamos que com as convenções de sinal aqui adotadas, o espaço $\mathfrak{X}^{\vee}(. M)$ dos campos multivetoriais sobre $M$, com o produto tensorial simetrizado $\vee$ como produto associativo e comutativo e com o colchete de Schouten como comutador, se torna uma álgebra. de Poisson, naturalmente associada à variedade $M$, que estende a álgebra de Lie $\mathfrak{X}(. M)$ dos campos vetoriais sobre $M$. (Uma álgebra de Poisson é uma álgebra equipada com um produto associativo e com um comutador que lhe confere a estrutura de uma álgebra de Lie, tal que, como condiçào de compatibilidade, vale a regra de Leibniz.) 


\section{Apêndice B}

\section{Formas de Conexão}

Neste apêndice, calculamos as expressões das derivadas covariantes dos campos vetoriais de Boyer-Lindquist e as derivadas covariantes dos campos vetoriais nulos $N^{ \pm}$.

Proposição B.1 As derivadas covariantes dos campos vetoriais de Boyer-Lindquist $E_{1}, E_{2}$, $E_{3}, E_{4}$, obtidas a partir das formas de conerão, pela fórmula

$$
\nabla_{E_{t}} E_{j}=\omega_{j}^{k}\left(E_{i}\right) E_{k},
$$

são [12, p. 95]:

$$
\begin{aligned}
& \nabla_{E_{1}} E_{1}=\epsilon \frac{a^{2} \sin \vartheta \cos \vartheta}{\rho^{3}} E_{2} \\
& \nabla_{E_{1}} E_{2}=-\frac{a^{2} \sin \vartheta \cos \vartheta}{\rho^{3}} E_{1} \\
& \nabla_{E_{2}} E_{1}=\frac{r \sqrt{|\Delta|}}{\rho^{3}} E_{2} \\
& \nabla_{E_{2}} E_{2}=-\epsilon \frac{r \sqrt{|\Delta|}}{\rho^{3}} E_{1} \text {, } \\
& \nabla_{E_{0}} E_{0}=F E_{1}-\epsilon \frac{a^{2} \sin \vartheta \cos \vartheta}{\rho^{3}} E_{2}, \\
& \nabla_{E_{0}} E_{3}=\nabla_{E_{3}} E_{0}=-\epsilon \frac{a r \sin \vartheta}{\rho^{3}} E_{1}-\epsilon \frac{a \cos \vartheta \sqrt{|\Delta|}}{\rho^{3}} E_{2}, \\
& \nabla_{E_{3}} E_{3}=-\epsilon \frac{r \sqrt{|\Delta|}}{\rho^{3}} E_{1}-\frac{\left(r^{2}+a^{2}\right) \cos \vartheta}{\rho^{3} \sin \vartheta} E_{2}, \\
& \nabla_{E_{0}} E_{1}=F E_{0}+\frac{a r \sin v}{\rho^{3}} E_{3}, \\
& \nabla_{E_{0}} E_{2}=-\frac{a^{2} \sin \vartheta \cos \vartheta}{\rho^{3}} E_{0}+\epsilon \frac{a \cos \vartheta \sqrt{|\Delta|}}{\rho^{3}} E_{3},
\end{aligned}
$$




$$
\begin{gathered}
\nabla_{E_{3}} E_{1}=-\epsilon \frac{a r \sin \vartheta}{\rho^{3}} E_{0}+\frac{r \sqrt{|\Delta|}}{\rho^{3}} E_{3}, \\
\nabla_{E_{3}} E_{2}=-\frac{a \cos \vartheta \sqrt{|\Delta|}}{\rho^{3}} E_{0}+\frac{\left(r^{2}+a^{2}\right) \cos \vartheta}{\rho^{3} \sin \vartheta} E_{3}, \\
\nabla_{E_{1}} E_{0}=-\frac{a r \sin \vartheta}{\rho^{3}} E_{3}, \\
\nabla_{E_{1}} E_{3}=-\epsilon \frac{a r \sin \vartheta}{\rho^{3}} E_{0}, \\
\nabla_{E_{2}} E_{0}=\epsilon \frac{a \cos \vartheta \sqrt{|\Delta|}}{\rho^{3}} E_{3}, \\
\nabla_{E_{2}} E_{3}=\frac{a \cos \vartheta \sqrt{|\Delta|}}{\rho^{3}} E_{0},
\end{gathered}
$$

onde usamos a abreviação

$$
F=\frac{\partial}{\partial r}\left(\frac{\sqrt{|\Delta|}}{\rho}\right)=\epsilon \frac{(r-m) \rho^{2}-r \Delta}{\rho^{3} \sqrt{|\Delta|}} .
$$

DEmonstraÇÃo. Lembremos que os campos referenciais ortonormais de Boyer-Lindquist sào $[12$, p. 90]

$$
\begin{array}{r}
E_{1}=\frac{\sqrt{|\Delta|}}{\rho} \partial_{r} . \quad E_{3}=\frac{W}{\rho \sin \vartheta} \\
E_{2}=\frac{1}{\rho} \partial_{\vartheta} . \quad E_{0}=\frac{V}{\rho \sqrt{|\Delta|}}
\end{array}
$$

e que os campos coreferenciais ortonormais Boyer-Lindquist são [12, p. 91]

$$
\begin{gathered}
\omega^{1}=\frac{\rho}{\sqrt{|\Delta|}} d r \quad, \quad \omega^{3}=\frac{\sin \vartheta}{\rho}\left(\left(r^{2}+a^{2}\right) d \varphi-a d t\right) \\
\omega^{2}=\rho d \vartheta \quad, \quad \omega^{0}=\frac{\sqrt{|\Delta|}}{\rho}\left(d t-a \sin ^{2} \vartheta d \varphi\right)
\end{gathered}
$$

Para calcularmos as formas de conexào dos campos referenciais de Boyer-Lindquist devemos achar inicialmente as derivadas exteriores $d \omega^{i}$ das 1 -formas duais acima. Para este cálculo é conveniente listar um conjunto de fórmulas de derivadas parciais:

$$
\begin{gathered}
\frac{\partial \rho}{\partial r}=\frac{r}{\rho}, \frac{\partial}{\partial r} \frac{1}{\rho}=-\frac{r}{\rho^{3}}, \frac{\partial \rho}{\partial \vartheta}=-\frac{a^{2} \sin \vartheta \cos \vartheta}{\rho}, \frac{\partial}{\partial \vartheta} \frac{1}{\rho}=\frac{a^{2} \sin \vartheta \cos \vartheta}{\rho^{3}}, \\
\frac{\partial \Delta}{\partial r}=2(r-m), \frac{\partial}{\partial r} \sqrt{|\Delta|}=\epsilon \frac{r-m}{\sqrt{|\Delta|}}, \frac{\partial}{\partial r} \frac{1}{\sqrt{|\Delta|}}=-\frac{r-m}{\Delta \sqrt{|\Delta|}} .
\end{gathered}
$$


O cálculo das derivadas dos doís primeiros campos torna-se imediato, enquanto que o cálculo das duas últimas derivadas procede como segue:

$$
\begin{aligned}
d \omega^{3}= & \frac{\partial}{\partial r}\left(\frac{1}{\rho}\right) \rho d r \wedge \omega^{3}+\frac{\partial}{\partial \vartheta}(\sin \vartheta) \frac{1}{\sin \vartheta} d \vartheta \wedge \omega^{3}+\frac{2 r \sin \vartheta}{\rho} d r \wedge d \varphi \\
= & -\frac{r \sqrt{|\Delta|}}{\rho^{3}} \omega^{1} \wedge \omega^{3}+\frac{\cos \vartheta}{\sin \vartheta} \frac{1}{\rho} \omega^{2} \wedge \omega^{3} \\
& +\frac{2 r \sin \vartheta \sqrt{|\Delta|}}{\rho^{2}} \omega^{1} \wedge\left(\frac{1}{\rho \sin \vartheta} \omega^{3}+\frac{a}{\rho \sqrt{|\Delta|}} \omega^{0}\right) \\
= & \frac{r \sqrt{|\Delta|}}{\rho^{3}} \omega^{1} \wedge \omega^{3}+\frac{\cos \vartheta}{\sin \vartheta} \frac{1}{\rho} \omega^{2} \wedge \omega^{3}+\frac{2 a r \sin \vartheta}{\rho^{3}} \omega^{1} \wedge \omega^{0}, \\
d \omega^{0}= & \frac{\partial}{\partial r}\left(\frac{\sqrt{|\Delta|}}{\rho}\right) \frac{\rho}{\sqrt{|\Delta|}} d r \wedge \omega^{0}+\frac{\partial}{\partial \vartheta}\left(\frac{1}{\rho}\right) \rho d \vartheta \wedge \omega^{0}-\frac{2 a \sin \vartheta \cos \vartheta \sqrt{|\Delta|}}{\rho} d \vartheta \wedge d \varphi \\
= & F \omega^{1} \wedge \omega^{0}+\frac{a^{2} \sin \vartheta \cos \vartheta}{\rho^{3}} \omega^{2} \wedge \omega^{0} \\
& -\frac{2 a \sin \vartheta \cos \vartheta \sqrt{|\Delta|}}{\rho^{2}} \omega^{2} \wedge\left(\frac{1}{\rho \sin \vartheta} \omega^{3}+\frac{a}{\rho \sqrt{|\Delta|}} \omega^{0}\right) \\
= & F \omega^{1} \wedge \omega^{0}-\frac{a^{2} \sin \vartheta \cos \vartheta}{\rho^{3}} \omega^{2} \wedge \omega^{0}-\frac{2 a \cos \vartheta \sqrt{|\Delta|}}{\rho^{3}} \omega^{2} \wedge \omega^{3} .
\end{aligned}
$$

O resultado para as derivadas exteriores é [12, p. 92]:

$$
\begin{gathered}
d \omega^{1}=\frac{a^{2} \sin \vartheta \cos \vartheta}{\rho^{3}} \omega^{1} \wedge \omega^{2}, \\
d \omega^{2}=\frac{r \sqrt{|\Delta|}}{\rho^{3}} \omega^{1} \wedge \omega^{2}, \\
d \omega^{3}=\frac{\left(r^{2}+a^{2}\right) \cos \vartheta}{\rho^{3} \sin \vartheta} \omega^{2} \wedge \omega^{3}-\frac{r \sqrt{|\Delta|}}{\rho^{3}} \omega^{3} \wedge \omega^{1}+\frac{2 a r \sin \vartheta}{\rho^{3}} \omega^{1} \wedge \omega^{0}, \\
d \omega^{0}=F \omega^{1} \wedge \omega^{0}-\frac{2 a \cos \vartheta \sqrt{|\Delta|}}{\rho^{3}} \omega^{2} \wedge \omega^{3}-\frac{a^{2} \sin \vartheta \cos \vartheta}{\rho^{3}} \omega^{2} \wedge \omega^{0} .
\end{gathered}
$$

A partir destas fórmulas, podemos calcular os coeficientes de conexão $\omega_{j}^{i}$, usando a primeira equação estrutural $[12$, p. 50]

$$
d \omega^{i}=-\omega_{j}^{i} \wedge \omega^{j}
$$

em conjunto com a equaçào $[12$, p. 5:2]

$$
\omega_{j}^{i}\left(E_{k}\right)=\frac{1}{2}\left(d \omega^{i}\left(E_{j}, E_{k}\right)+\epsilon_{i} \epsilon_{j} d \omega^{j}\left(E_{k}, E_{i}\right)-\epsilon_{i} \epsilon_{k} d \omega^{k}\left(E_{i}, E_{j}\right)\right)
$$


(não somando sobre $i . j$ ou $k$ ): com

$$
\epsilon_{1}=\epsilon, \epsilon_{2}=1, \epsilon_{3}=1, \epsilon_{0}=-\epsilon .
$$

Desta forma. temos

- $i=1, j=2$ :

$$
\omega_{2}^{1}\left(E_{k}\right)=\frac{1}{2}\left(d \omega^{1}\left(E_{2}, E_{k}\right)+\epsilon d \omega^{2}\left(E_{k}, E_{1}\right)-\epsilon \epsilon_{k} d \omega^{k}\left(E_{1}, E_{2}\right)\right) .
$$

O primeiro termo é diferente de zero somente para $k=1$, o segundo termo é diferente de zero somente para $k=2$ e o terceiro termo é diferente de zero somente para $k=1$ ou $k=2$, resultando em

$$
\begin{gathered}
\omega_{2}^{1}\left(E_{1}\right)=\frac{1}{2}\left(d \omega^{1}\left(E_{2}, E_{1}\right)-d \omega^{1}\left(E_{1}, E_{2}\right)\right)=-d \omega^{1}\left(E_{1}, E_{2}\right), \\
\omega_{2}^{1}\left(E_{2}\right)=\frac{1}{2}\left(\epsilon d \omega^{2}\left(E_{2}, E_{1}\right)-\epsilon d \omega^{2}\left(E_{1}, E_{2}\right)\right)=-\epsilon d \omega^{2}\left(E_{1}, E_{2}\right) .
\end{gathered}
$$

- $i=1, j=3$ :

$$
\omega_{3}^{1}\left(E_{k}\right)=\frac{1}{2}\left(d \omega^{1}\left(E_{3}, E_{k}\right)+\epsilon d \omega^{3}\left(E_{k}, E_{1}\right)-\epsilon \epsilon_{k} d \omega^{k}\left(E_{1}, E_{3}\right)\right) .
$$

O primeiro termo é sempre zero, o segundo termo é diferente de zero somente para $k=3$ ou $k=0$ e o terceiro termo é diferente de zero somente para $k=3$, resultando em

$$
\begin{gathered}
\omega_{3}^{1}\left(E_{3}\right)=\frac{1}{2}\left(\epsilon d \omega^{3}\left(E_{3}, E_{1}\right)-\epsilon d \omega^{3}\left(E_{1}, E_{3}\right)\right)=-\epsilon d \omega^{3}\left(E_{1}, E_{3}\right), \\
\omega_{3}^{1}\left(E_{0}\right)=\frac{1}{2} \epsilon d \omega^{3}\left(E_{0}, E_{1}\right) .
\end{gathered}
$$

- $i=1, j=0$ :

$$
\omega_{0}^{1}\left(E_{k}\right)=\frac{1}{2}\left(d \omega^{1}\left(E_{0}, E_{k}\right)-d \omega^{0}\left(E_{k}, E_{1}\right)-\epsilon \epsilon_{k} d \omega^{k}\left(E_{1}, E_{0}\right)\right) .
$$

O primeiro termo é sempre zero, o segundo termo é diferente de zero somente para $k=0$ e o terceiro termo é diferente de zero somente para $k=3$ ou $k=0$, resultando em

$$
\begin{gathered}
\omega_{0}^{1}\left(E_{3}\right)=-\frac{1}{2} \epsilon d \omega^{3}\left(E_{1}, E_{0}\right), \\
\omega_{0}^{1}\left(E_{0}\right)=-\frac{1}{2}\left(d \omega^{0}\left(E_{0}, E_{1}\right)-d \omega^{0}\left(E_{1}, E_{0}\right)\right)=d \omega^{0}\left(E_{1}, E_{0}\right) .
\end{gathered}
$$

- $i=2, j=3$ :

$$
\omega_{3}^{2}\left(E_{k}\right)=\frac{1}{2}\left(d \omega^{2}\left(E_{3}, E_{k}\right)+d \omega^{3}\left(E_{k}, E_{2}\right)-\epsilon_{k} d \omega^{k}\left(E_{2}, E_{3}\right)\right) .
$$

O primeiro termo é sempre zero, o segundo termo é diferente de zero somente para $k=3$ e o terceiro termo é diferente de zero somente para $k=3$ ou $k=0$, resultando em

$$
\begin{gathered}
\omega_{3}^{2}\left(E_{3}\right)=\frac{1}{2}\left(d \omega^{3}\left(E_{3}, E_{2}\right)-d \omega^{3}\left(E_{2}, E_{3}\right)\right)=-d \omega^{3}\left(E_{2}, E_{3}\right), \\
\omega_{3}^{2}\left(E_{0}\right)=\frac{1}{2} \epsilon d \omega^{0}\left(E_{2}, E_{3}\right) .
\end{gathered}
$$


- $i=2, j=0$ :

$$
\omega_{0}^{2}\left(E_{k}\right)=\frac{1}{2}\left(d \omega^{2}\left(E_{0}, E_{k}\right)-\epsilon d \omega^{0}\left(E_{k}, E_{2}\right)-\epsilon_{k} d \omega^{k}\left(E_{2}, E_{0}\right)\right) .
$$

O primeiro termo é sempre zero, o segundo termo é diferente de zero somente para $k=3$ ou $k=0$ e o terceiro termo é diferente de zero somente para $k=0$, resultando em

$$
\begin{gathered}
\omega_{0}^{2}\left(E_{3}\right)=-\frac{1}{2} \epsilon d \omega^{0}\left(E_{3}, E_{2}\right) . \\
\omega_{0}^{2}\left(E_{0}\right)=-\frac{1}{2} \epsilon\left(d \omega^{0}\left(E_{0}, E_{2}\right)-d \omega^{0}\left(E_{2}, E_{0}\right)\right)=\epsilon d \omega^{0}\left(E_{2}, E_{0}\right) .
\end{gathered}
$$

- $i=3, j=0$ :

$$
\omega_{0}^{3}\left(E_{k}\right)=\frac{1}{2}\left(d \omega^{3}\left(E_{0}, E_{k}\right)-\epsilon d \omega^{0}\left(E_{k}, E_{3}\right)-\epsilon_{k} d \omega^{k}\left(E_{3}, E_{0}\right)\right) .
$$

O primeiro termo é diferente de zero somente para $k=1$. o segundo termo é diferente de zero somente para $k=2$ e o terceiro termo é sempre zero, resultando em

$$
\begin{gathered}
\omega_{0}^{3}\left(E_{1}\right)=\frac{1}{2} d \omega^{3}\left(E_{0}, E_{1}\right), \\
\omega_{0}^{3}\left(E_{2}\right)=-\frac{1}{2} \epsilon d \omega^{0}\left(E_{2}, E_{3}\right) .
\end{gathered}
$$

Obtemos portanto o seguinte resultado [12, p. 92]:

$$
\begin{gathered}
\omega_{2}^{1}=-\frac{a^{2} \sin \vartheta \cos \vartheta}{\rho^{3}} \omega^{1}-\epsilon \frac{r \sqrt{|\Delta|}}{\rho^{3}} \omega^{2}, \\
\omega_{3}^{1}=-\epsilon \frac{r \sqrt{|\Delta|}}{\rho^{3}} \omega^{3}-\epsilon \frac{a r \sin \vartheta}{\rho^{3}} \omega^{0}, \\
\omega_{0}^{1}=-\epsilon \frac{a r \sin \vartheta}{\rho^{3}} \omega^{3}+F \omega^{0}, \\
\omega_{3}^{2}=-\frac{\left(r^{2}+a^{2}\right) \cos \vartheta}{\rho^{3} \sin \vartheta} \omega^{3}-\epsilon \frac{a \cos \vartheta \sqrt{|\Delta|}}{\rho^{3}} \omega^{0}, \\
\omega_{0}^{2}=-\epsilon \frac{a \cos \vartheta \sqrt{|\Delta|}}{\rho^{3}} \omega^{3}-\epsilon \frac{a^{2} \sin \vartheta \cos \vartheta}{\rho^{3}} \omega^{0}, \\
\omega_{0}^{3}=-\frac{a r \sin \vartheta}{\rho^{3}} \omega^{1}+\epsilon \frac{a \cos \vartheta \sqrt{|\Delta|}}{\rho^{3}} \omega^{2} .
\end{gathered}
$$

Usando agora a fórmula

$$
\nabla_{E_{i}} E_{j}=\omega_{j}^{k}\left(E_{i}\right) E_{k}
$$

concluímos a demonstração.

Lembremos que os dois campos vetorias nulos são [12, pp. 79.95]

$$
N_{ \pm}= \pm \partial_{r}+\Delta^{-1} V=\frac{\rho}{\sqrt{|\Delta|}}\left(\epsilon E_{0} \pm E_{1}\right)
$$


e que as um-formas duais. determinadas pela condição

$$
N^{ \pm}\left(E_{i}\right)=g\left(N_{ \pm}, E_{i}\right),
$$

são

$$
N^{ \pm}=\frac{\rho}{\sqrt{|\Delta|}}\left(-\omega^{0} \pm \epsilon \omega^{1}\right)
$$

Proposição B.2 As expressões das derivadas covariantes dos campos vetoriais nulos $N^{ \pm}$ são

$$
\begin{gathered}
\nabla_{E_{1}} N^{ \pm}=-F N^{ \pm} \pm \epsilon \frac{a^{2} \sin \vartheta \cos \vartheta}{\rho^{2} \sqrt{|\Delta|}} \omega^{2}-\epsilon \frac{a r \sin \vartheta}{\rho^{2} \sqrt{|\Delta|}} \omega^{3}, \\
\nabla_{E_{2}} N^{ \pm}=-\frac{a^{2} \sin \vartheta \cos \vartheta}{\rho^{3}} N^{ \pm} \pm \frac{r}{\rho^{2}} \omega^{2}+\frac{a \cos \vartheta}{\rho^{2}} \omega^{3}, \\
\nabla_{E_{3}} N^{ \pm}=\mp \frac{a r \sin \vartheta}{\rho^{3}} N^{ \pm}-\frac{a \cos \vartheta}{\rho^{2}} \omega^{2} \pm \frac{r}{\rho^{2}} \omega^{3}, \\
\nabla_{E_{0}} N^{ \pm}= \pm \epsilon F N^{ \pm}-\frac{a^{2} \sin \vartheta \cos \vartheta}{\rho^{2} \sqrt{|\Delta|}} \omega^{2} \pm \frac{a r \sin \vartheta}{\rho^{2} \sqrt{|\Delta|}} \omega^{3} .
\end{gathered}
$$

DemonstraÇÃo. Para esta demonstração deveremos calcular explicitamente a expressão

$$
\left(\nabla_{E_{k}} N^{ \pm}\right)\left(E_{i}\right)=\mathfrak{L}_{E_{k}}\left(N^{ \pm}\left(E_{i}\right)\right)-N^{ \pm}\left(\nabla_{E_{k}} E_{i}\right)
$$

Para iniciarmos o cálculo das expressões relacionadas ao primeiro termo da equação acima, observemos que o número de casos pode ser reduzido observando que as expressões $L_{E_{k}}\left(N^{ \pm}\left(E_{i}\right)\right)$ são zero quando $i=2$ ou $i=3$ ou $k=3$ ou $k=0$, enquanto que

$$
\begin{gathered}
\mathfrak{L}_{E_{1}}\left(N^{ \pm}\left(E_{1}\right)\right)= \pm \epsilon \frac{\sqrt{|\Delta|}}{\rho} \frac{\partial}{\partial r}\left(\frac{\rho}{\sqrt{|\Delta|}}\right)=\mp \epsilon \frac{\rho F}{\sqrt{|\Delta|}}, \\
\mathfrak{L}_{E_{1}}\left(N^{ \pm}\left(E_{0}\right)\right)=-\frac{\sqrt{|\Delta|}}{\rho} \frac{\partial}{\partial r}\left(\frac{\rho}{\sqrt{|\Delta|}}\right)=\frac{\rho F}{\sqrt{|\Delta|}}, \\
\mathfrak{L}_{E_{2}}\left(N^{ \pm}\left(E_{1}\right)\right)= \pm \epsilon \frac{1}{\rho} \frac{\partial}{\partial \vartheta}\left(\frac{\rho}{\sqrt{|\Delta|}}\right)=\mp \epsilon \frac{a^{2} \sin \vartheta \cos \vartheta}{\rho^{2} \sqrt{|\Delta|}}, \\
\mathfrak{L}_{E_{2}}\left(N^{ \pm}\left(E_{0}\right)\right)=-\frac{1}{\rho} \frac{\partial}{\partial \vartheta}\left(\frac{\rho}{\sqrt{|\Delta|}}\right)=\frac{a^{2} \sin \vartheta \cos \vartheta}{\rho^{2} \sqrt{|\Delta|}} .
\end{gathered}
$$

Para o cálculo das expressòes relacionadas ao segundo termo, distinguimos os seguintes casos: 
- $k=1$ :

A expressào $\Gamma_{E_{1}} E_{i}$ nào possue componentes ao longo de $E_{0}$ ou de $E_{1}$ quando $i=1$ ou $i=0$, portanto

$$
\begin{gathered}
-N^{ \pm}\left(\nabla_{E_{1}} E_{2}\right)=\frac{\rho}{\sqrt{|\Delta|}}\left(\omega^{0} \mp \epsilon \omega^{1}\right)\left(\nabla_{E_{1}} E_{2}\right)= \pm \epsilon \frac{a^{2} \sin \vartheta \cos \vartheta}{\rho^{2} \sqrt{|\Delta|}}, \\
-V^{ \pm}\left(\nabla_{E_{1}} E_{3}\right)=\frac{\rho}{\sqrt{|\Delta|}}\left(\omega^{0} \mp \epsilon \omega^{1}\right)\left(\nabla_{E_{1}} E_{3}\right)=-\epsilon \frac{a r \sin \vartheta}{\rho^{2} \sqrt{|\Delta|}} .
\end{gathered}
$$

- $k=2$ :

A expressão $\Gamma_{E_{2}} E_{i}$ nào possue componentes ao longo de $E_{0}$ ou de $E_{1}$ quando $i=1$ ou $i=0$, portanto

$$
\begin{aligned}
& -N^{ \pm}\left(\nabla_{E_{2}} E_{2}\right)=\frac{\rho}{\sqrt{|\Delta|}}\left(\omega^{0} \mp \epsilon \omega^{1}\right)\left(\nabla_{E_{2}} E_{2}\right)= \pm \frac{r}{\rho^{2}} \\
& -N^{ \pm}\left(\nabla_{E_{2}} E_{3}\right)=\frac{\rho}{\sqrt{|\Delta|}}\left(\omega^{0} \mp \epsilon \omega^{1}\right)\left(\nabla_{E_{2}} E_{3}\right)=\frac{a \cos \vartheta}{\rho^{2}} .
\end{aligned}
$$

- $k=3$ :

$$
\begin{gathered}
-V^{ \pm}\left(\nabla_{E_{3}} E_{1}\right)=\frac{\rho}{\sqrt{|\Delta|}}\left(\omega^{0} \mp \epsilon \omega^{1}\right)\left(\nabla_{E_{3}} E_{1}\right)=-\epsilon \frac{a r \sin \vartheta}{\rho^{2} \sqrt{|\Delta|}}, \\
-N^{ \pm}\left(\nabla_{E_{3}} E_{2}\right)=\frac{\rho}{\sqrt{|\Delta|}}\left(\omega^{0} \mp \epsilon \omega^{1}\right)\left(\nabla_{E_{3}} E_{2}\right)=-\frac{a \cos \vartheta}{\rho^{2}}, \\
-N^{ \pm}\left(\nabla_{E_{3}} E_{3}\right)=\frac{\rho}{\sqrt{|\Delta|}}\left(\omega^{0} \mp \epsilon \omega^{1}\right)\left(\nabla_{E_{3}} E_{3}\right)= \pm \frac{r}{\rho^{2}}, \\
-V^{ \pm}\left(\nabla_{E_{3}} E_{0}\right)=\frac{\rho}{\sqrt{|\Delta|}}\left(\omega^{0} \mp \epsilon \omega^{1}\right)\left(\nabla_{E_{3}} E_{0}\right)= \pm \frac{a r \sin \vartheta}{\rho^{2} \sqrt{|\Delta|}} .
\end{gathered}
$$

- $k=0$ :

$$
\begin{gathered}
-N^{ \pm}\left(\nabla_{E_{0}} E_{1}\right)=\frac{\rho}{\sqrt{|\Delta|}}\left(\omega^{0} \mp \epsilon \omega^{1}\right)\left(\nabla_{E_{0}} E_{1}\right)=\frac{\rho F}{\sqrt{|\Delta|}}, \\
-N^{ \pm}\left(\nabla_{E_{0}} E_{2}\right)=\frac{\rho}{\sqrt{|\Delta|}}\left(\omega^{0} \mp \epsilon \omega^{1}\right)\left(\nabla_{E_{0}} E_{2}\right)=-\frac{a^{2} \sin \vartheta \cos \vartheta}{\rho^{2} \sqrt{|\Delta|}}, \\
-V^{ \pm}\left(\nabla_{E_{0}} E_{3}\right)=\frac{\rho}{\sqrt{|\Delta|}}\left(\omega^{0} \mp \epsilon \omega^{1}\right)\left(\nabla_{E_{0}} E_{3}\right)= \pm \frac{a r \sin \vartheta}{\rho^{2} \sqrt{|\Delta|}}, \\
-.^{ \pm}\left(\nabla_{E_{0}} E_{0}\right)=\frac{\rho}{\sqrt{|\Delta|}}\left(\omega^{0} \mp \epsilon \omega^{1}\right)\left(\nabla_{E_{0}} E_{0}\right)=\mp \epsilon \frac{\rho}{\sqrt{|\Delta|}} F .
\end{gathered}
$$

concluíndo a demonstração. 


\section{Apêndice C}

\section{O Tensor de Killing}

Lembremos que o tensor de Kïlling na solução de lierr tem componentes contravariantes

$$
\Lambda^{-i j}=\frac{\Delta}{2}\left(N_{+}^{i} N_{-}^{j}+N_{+}^{j} N_{-}^{i}\right)+r^{2} g^{i j} .
$$

e componentes covariantes

$$
K_{i j}=\frac{\Delta}{2}\left(N_{i}^{+} N_{j}^{-}+N_{j}^{+} N_{i}^{-}\right)+r^{2} g_{i j} .
$$

Para demonstrarmos que $K^{\prime}$ é um tensor de Killing basta mostrar que satisfaz a equação de Killing

$$
S_{k i j}=\nabla_{E_{k}} K_{i j}+\nabla_{E_{i}} K_{j k}+\nabla_{E_{j}} K_{k i}=0,
$$

que calculamos a seguir, lembrando que $N_{i}^{ \pm}=0$ se $i=2$ ou $i=3$ :

$$
\begin{aligned}
\frac{1}{3} S_{111} & =\nabla_{E_{1}} K_{11} \\
& =\mathfrak{L}_{E_{1}}(\Delta) N_{1}^{+} N_{1}^{-}+\Delta\left(\nabla_{E_{1}} N^{+}\right)_{1} N_{1}^{-}+\Delta N_{1}^{+}\left(\nabla_{E_{1}} N^{-}\right)_{1}+\mathfrak{L}_{E_{1}}\left(r^{2}\right) g_{11} \\
& =\frac{\sqrt{|\Delta|}}{\rho} \frac{\partial \Delta}{\partial r} N_{1}^{+} N_{1}^{-}-2 F \Delta N_{1}^{+} N_{1}^{-}+\frac{\sqrt{|\Delta|}}{\rho} 2 \epsilon r \\
& =-\frac{\sqrt{|\Delta|}}{\rho} 2(r-M) \frac{\rho^{2}}{|\Delta|}+2 \Delta \frac{\rho^{2} F}{|\Delta|}+\frac{\sqrt{|\Delta|}}{\rho} 2 \epsilon r \\
& =0, \\
\frac{1}{3} S_{222} & =\nabla_{E_{2}} K_{22} \\
& =\mathfrak{L}_{E_{2}}(\Delta) N_{2}^{+} N_{2}^{-}+\Delta\left(\nabla_{E_{2}} N^{+}\right)_{2} N_{2}^{-}+\Delta N_{2}^{+}\left(\nabla_{E_{2}} N^{-}\right)_{2}+\mathfrak{L}_{E_{2}}\left(r^{2}\right) g_{22} \\
& =0,
\end{aligned}
$$




$$
\begin{aligned}
& \frac{1}{3} S_{333}=\nabla_{E_{3}} K_{33} \\
& =\mathfrak{L}_{E_{3}}(\Delta) N_{3}^{+} N_{3}^{-}+\Delta\left(\nabla_{E_{3}} N^{+}\right)_{3} N_{3}^{-}+\Delta N_{3}^{+}\left(\nabla_{E_{3}} N^{-}\right)_{3}+\mathfrak{L}_{E_{3}}\left(r^{2}\right) g_{33} \\
& =0 \text {, } \\
& \frac{1}{3} S_{000}=\Gamma_{E_{0}} K_{00} \\
& =\mathfrak{L}_{E_{0}}(\Delta) N_{0}^{+} N_{0}^{-}+\Delta\left(\nabla_{E_{0}} N^{+}\right)_{0} N_{0}^{-}+\Delta N_{0}^{+}\left(\nabla_{E_{0}} N^{-}\right)_{0}+\mathfrak{L}_{E_{0}}\left(r^{2}\right) g_{00} \\
& =\epsilon F \Delta N_{0}^{+} N_{0}^{-}-\epsilon F \Delta N_{0}^{+} N_{0}^{-} \\
& =0 \text {, } \\
& S_{122}=\nabla_{E_{1}} K_{22}+2 \nabla_{E_{2}} K_{12} \\
& =\mathfrak{\sim}_{E_{1}}(\Delta) N_{2}^{+} N_{2}^{-}+\Delta\left(\nabla_{E_{1}} N^{+}\right)_{2} N_{2}^{-}+\Delta N_{2}^{+}\left(\nabla_{E_{1}} N^{-}\right)_{2}+\mathfrak{L}_{E_{1}}\left(r^{2}\right) g_{22} \\
& +\mathfrak{L}_{E_{2}}(\Delta) N_{1}^{+} N_{2}^{-}+\Delta\left(\nabla_{E_{2}} N^{+}\right)_{1}, V_{2}^{-}+\Delta N_{1}^{+}\left(\nabla_{E_{2}} N^{-}\right)_{2} \\
& +\mathfrak{L}_{E_{2}}(\Delta) N_{2}^{+} N_{1}^{-}+\Delta\left(\nabla_{E_{2}} N^{+}\right)_{2} \cdot V_{1}^{-}+\Delta N_{2}^{+}\left(\nabla_{E_{2}} N^{-}\right)_{1} \\
& +2 \mathfrak{\Omega}_{E_{2}}\left(r^{2}\right) g_{12} \\
& =\frac{\sqrt{|\Delta|}}{\rho} 2 r+\Delta\left(\epsilon \frac{\rho}{\sqrt{|\Delta|}}\right)\left(-\frac{r}{\rho^{2}}\right)+\Delta\left(\frac{r}{\rho^{2}}\right)\left(-\epsilon \frac{\rho}{\sqrt{|\Delta|}}\right) \\
& =0, \\
& S_{211}=\nabla_{E_{2}} K_{11}+2 \nabla_{E_{1}} K_{12} \\
& =\mathfrak{L}_{E_{2}}(\Delta) N_{1}^{+} N_{1}^{-}+\Delta\left(\nabla_{E_{2}} N^{+}\right)_{1} N_{1}^{-}+\Delta N_{1}^{+}\left(\nabla_{E_{2}} N^{-}\right)_{1}+\mathfrak{L}_{E_{2}}\left(r^{2}\right) g_{11} \\
& +\mathfrak{L}_{E_{1}}(\Delta) N_{1}^{+} N_{2}^{-}+\Delta\left(\nabla_{E_{1}} N^{+}\right)_{1} \cdot V_{2}^{-}+\Delta N_{1}^{+}\left(\nabla_{E_{1}} N^{-}\right)_{2} \\
& +\mathfrak{L}_{E_{1}}(\Delta) N_{2}^{+} N_{1}^{-}+\Delta\left(\nabla_{E_{1}} N^{+}\right)_{2} \cdot N_{1}^{-}+\Delta N_{2}^{+}\left(\nabla_{E_{1}} N^{-}\right)_{1} \\
& +2 \Omega_{E_{1}}\left(r^{2}\right) g_{12} \\
& =\Delta\left(-\epsilon \frac{a^{2} \sin \vartheta \cos \vartheta}{\rho^{2} \sqrt{|\Delta|}}\right)\left(-\epsilon \frac{\rho}{\sqrt{|\Delta|}}\right)+\Delta\left(\epsilon \frac{\rho}{\sqrt{|\Delta|}}\right)\left(\epsilon \frac{a^{2} \sin \vartheta \cos \vartheta}{\rho^{2} \sqrt{|\Delta|}}\right) \\
& +\Delta\left(\epsilon \frac{\rho}{\sqrt{|\Delta|}}\right)\left(-\epsilon \frac{a^{2} \sin \vartheta \cos \vartheta}{\rho^{2} \sqrt{|\Delta|}}\right)+\Delta\left(\epsilon \frac{a^{2} \sin \vartheta \cos \vartheta}{\rho^{2} \sqrt{|\Delta|}}\right)\left(-\epsilon \frac{\rho}{\sqrt{|\Delta|}}\right) \\
& =0, \\
& S_{133}=\nabla_{E_{1}} K_{33}+2 \nabla_{E_{3}} K_{13} \\
& =\mathfrak{L}_{E_{1}}(\Delta) N_{3}^{+} N_{3}^{-}+\Delta\left(\nabla_{E_{1}} N^{+}\right)_{3} N_{3}^{-}+\Delta N_{3}^{+}\left(\nabla_{E_{1}} N^{-}\right)_{3}+\mathfrak{L}_{E_{1}}\left(r^{2}\right) g_{33} \\
& +\mathfrak{L}_{E_{3}}(\Delta) N_{1}^{+} N_{3}^{-}+\Delta\left(\nabla_{E_{2}} N^{+}\right)_{1} \cdot V_{3}^{-}+\Delta N_{1}^{+}\left(\nabla_{E_{3}} N^{-}\right)_{3} \\
& +\mathfrak{L}_{E_{3}}(\Delta) N_{3}^{+} N_{1}^{-}+\Delta\left(\nabla_{E_{3}} N^{+}\right)_{3} \cdot V_{1}^{-}+\Delta N_{3}^{+}\left(\nabla_{E_{3}} N^{-}\right)_{1} \\
& +2 \mathfrak{L}_{E_{3}}\left(r^{2}\right) g_{13}
\end{aligned}
$$




$$
\begin{aligned}
& =\frac{\sqrt{|\Delta|}}{\rho} 2 r+\Delta\left(\epsilon \frac{\rho}{\sqrt{|\Delta|}}\right)\left(-\frac{r}{\rho^{2}}\right)+\Delta\left(\frac{r}{\rho^{2}}\right)\left(-\epsilon \frac{\rho}{\sqrt{|\Delta|}}\right) \\
& =0 \text {. } \\
& S_{311}=\nabla_{E_{3}} K_{11}+2 \nabla_{E_{1}} K_{13} \\
& =\mathfrak{L}_{E_{3}}(\Delta) N_{1}^{+} N_{1}^{-}+\Delta\left(\nabla_{E_{3}} N^{+}\right)_{1} N_{1}^{-}+\Delta N_{1}^{+}\left(\nabla_{E_{3}} N^{-}\right)_{1}+\mathfrak{L}_{E_{3}}\left(r^{2}\right) g_{11} \\
& +\mathfrak{L}_{E_{1}}(\Delta) N_{1}^{+} N_{3}^{-}+\Delta\left(\nabla_{E_{1}} V^{+}\right)_{1} N_{3}^{-}+\Delta N_{1}^{+}\left(\nabla_{E_{1}} N^{-}\right)_{3} \\
& +\mathfrak{L}_{E_{1}}(\Delta) N_{3}^{+} N_{1}^{-}+\Delta\left(\nabla_{E_{1}} V^{+}\right)_{3} N_{1}^{-}+\Delta N_{3}^{+}\left(\nabla_{E_{1}} N^{-}\right)_{1} \\
& +2 \mathfrak{L}_{E_{1}}\left(r^{2}\right) g_{13} \\
& =-\frac{\operatorname{ar} \sin \vartheta}{\rho^{3}} \Delta N_{1}^{+} N_{1}^{-}+\frac{\operatorname{arsin} \vartheta}{\rho^{3}} \Delta N_{1}^{+} N_{1}^{-} \\
& +\Delta\left(\epsilon \frac{\rho}{\sqrt{|\Delta|}}\right)\left(-\epsilon \frac{a r \sin \vartheta}{\rho^{2} \sqrt{|\Delta|}}\right)+\Delta\left(-\epsilon \frac{a r \sin \vartheta}{\rho^{2} \sqrt{|\Delta|}}\right)\left(-\epsilon \frac{\rho}{\sqrt{|\Delta|}}\right) \\
& =0 \text {, } \\
& S_{100}=\nabla_{E_{1}} K_{00}+2 \nabla_{E_{0}} K_{10} \\
& =\mathfrak{L}_{E_{1}}(\Delta) N_{0}^{+} N_{0}^{-}+\Delta\left(\nabla_{E_{1}} N^{+}\right)_{0} N_{0}^{-}+\Delta N_{0}^{+}\left(\nabla_{E_{1}} N^{-}\right)_{0}+\mathfrak{L}_{E_{1}}\left(r^{2}\right) g_{00} \\
& +\mathfrak{L}_{E_{0}}(\Delta) N_{1}^{+} N_{0}^{-}+\Delta\left(\nabla_{E_{0}} N^{+}\right)_{1} N_{0}^{-}+\Delta N_{1}^{+}\left(\nabla_{E_{0}} N^{-}\right)_{0} \\
& +\mathfrak{L}_{E_{0}}(\Delta) N_{0}^{+} N_{1}^{-}+\Delta\left(\nabla_{E_{0}} V^{+}\right)_{0} N_{1}^{-}+\Delta N_{0}^{+}\left(\nabla_{E_{0}} N^{-}\right)_{1} \\
& +2 \mathfrak{L}_{E_{0}}\left(r^{2}\right) g_{10} \\
& =\frac{\sqrt{|\Delta|}}{\rho} \frac{\partial \Delta}{\partial r} N_{0}^{+} N_{0}^{-}-2 F \Delta N_{0}^{+} N_{0}^{-}-\frac{\sqrt{|\Delta|}}{\rho} 2 \epsilon r \\
& +\epsilon F \Delta N_{1}^{+} N_{0}^{-}-\epsilon F \Delta N_{1}^{+} N_{0}^{-}+\epsilon F \Delta N_{0}^{+} N_{1}^{-}-\epsilon F \Delta N_{0}^{+} N_{1}^{-} \\
& =\frac{\sqrt{|\Delta|}}{\rho} 2(r-M) \frac{\rho^{2}}{|\Delta|}-2 \Delta \frac{\rho^{2} F}{|\Delta|}-\frac{\sqrt{|\Delta|}}{\rho} 2 \epsilon r \\
& =0 \text {, } \\
& S_{011}=\nabla_{E_{0}} K_{11}+2 \nabla_{E_{1}} K_{10} \\
& =\mathfrak{L}_{E_{0}}(\Delta) N_{1}^{+} N_{1}^{-}+\Delta\left(\nabla_{E_{0}} N^{+}\right)_{1} N_{1}^{-}+\Delta N_{1}^{+}\left(\nabla_{E_{0}} N^{-}\right)_{1}+\mathfrak{L}_{E_{0}}\left(r^{2}\right) g_{11} \\
& +\mathfrak{L}_{E_{1}}(\Delta) N_{1}^{+} N_{0}^{-}+\Delta\left(\nabla_{E_{1}} \cdot V^{+}\right)_{1} N_{0}^{-}+\Delta N_{1}^{+}\left(\nabla_{E_{1}} N^{-}\right)_{0} \\
& +\mathfrak{L}_{E_{1}}(\Delta) N_{0}^{+} N_{1}^{-}+\Delta\left(\nabla_{E_{1}} V^{+}\right)_{0} N_{1}^{-}+\Delta N_{0}^{+}\left(\nabla_{E_{1}} N^{-}\right)_{1} \\
& +2 \mathfrak{L}_{E_{1}}\left(r^{2}\right) g_{10} \\
& =\epsilon F \Delta N_{1}^{+} N_{1}^{-}-\epsilon F \Delta N_{1}^{+} N_{1}^{-} \\
& +\frac{\sqrt{|\Delta|}}{\rho} \frac{\partial \Delta}{\partial r} N_{1}^{+} N_{0}^{-}-2 F \Delta N_{1}^{+} N_{0}^{-}+\frac{\sqrt{|\Delta|}}{\rho} \frac{\partial \Delta}{\partial r} N_{0}^{+} N_{1}^{-}-2 F \Delta N_{0}^{+} N_{1}^{-}
\end{aligned}
$$




$$
\begin{aligned}
& =\left(\frac{\sqrt{|\Delta|}}{\rho} 2(r-M)-2 F \Delta\right)\left(N_{1}^{+} N_{0}^{-}+N_{0}^{+} N_{1}^{-}\right) \\
& =0 \text {, } \\
& S_{233}=\nabla_{E_{2}} K_{33}+2 \nabla_{E_{3}} K_{23} \\
& =\mathfrak{L}_{E_{2}}(\Delta) N_{3}^{+} N_{3}^{-}+\Delta\left(\nabla_{E_{2}} N^{+}\right)_{3} N_{3}^{-}+\Delta N_{3}^{+}\left(\nabla_{E_{2}} N^{-}\right)_{3}+\mathfrak{L}_{E_{2}}\left(r^{2}\right) g_{33} \\
& +\mathfrak{L}_{E_{3}}(\Delta) N_{2}^{+} N_{3}^{-}+\Delta\left(\nabla_{E_{3}} N^{+}\right)_{2} N_{3}^{-}+\Delta N_{2}^{+}\left(\nabla_{E_{3}} N^{-}\right)_{3} \\
& +\mathfrak{L}_{E_{3}}(\Delta) N_{3}^{+} N_{2}^{-}+\Delta\left(\nabla_{E_{3}} N^{+}\right)_{3} N_{2}^{-}+\Delta N_{3}^{+}\left(\nabla_{E_{3}} N^{-}\right)_{2} \\
& +2 \mathfrak{L}_{E_{3}}\left(r^{2}\right) g_{23} \\
& =0 \text {, } \\
& S_{322}=\nabla_{E_{3}} K_{22}+2 \nabla_{E_{2}} K_{23} \\
& =\mathfrak{L}_{E_{3}}(\Delta) N_{2}^{+} N_{2}^{-}+\Delta\left(\nabla_{E_{3}} N^{+}\right)_{2} N_{2}^{-}+\Delta N_{2}^{+}\left(\nabla_{E_{3}} N^{-}\right)_{2}+\mathfrak{L}_{E_{3}}\left(r^{2}\right) g_{22} \\
& +\mathfrak{L}_{E_{2}}(\Delta) N_{2}^{+} N_{3}^{-}+\Delta\left(\nabla_{E_{2}} N^{+}\right)_{2} N_{3}^{-}+\Delta N_{2}^{+}\left(\nabla_{E_{2}} N^{-}\right)_{3} \\
& +\mathfrak{L}_{E_{2}}(\Delta) N_{3}^{+} N_{2}^{-}+\Delta\left(\nabla_{E_{2}} N^{+}\right)_{3} N_{2}^{-}+\Delta N_{3}^{+}\left(\nabla_{E_{2}} N^{-}\right)_{2} \\
& +2 \mathfrak{L}_{E_{2}}\left(r^{2}\right) g_{23} \\
& =0 \text {, } \\
& S_{200}=\nabla_{E_{2}} K_{00}+2 \nabla_{E_{0}} K_{20} \\
& =\mathfrak{L}_{E_{2}}(\Delta) N_{0}^{+} N_{0}^{-}+\Delta\left(\nabla_{E_{2}} N^{+}\right)_{0} N_{0}^{-}+\Delta N_{0}^{+}\left(\nabla_{E_{2}} N^{-}\right)_{0}+\mathfrak{L}_{E_{2}}\left(r^{2}\right) g_{00} \\
& +\mathfrak{L}_{E_{0}}(\Delta) N_{2}^{+} N_{0}^{-}+\Delta\left(\nabla_{E_{0}} N^{+}\right)_{2} N_{0}^{-}+\Delta N_{2}^{+}\left(\nabla_{E_{0}} N^{-}\right)_{0} \\
& +\mathfrak{L}_{E_{0}}(\Delta) N_{0}^{+} N_{2}^{-}+\Delta\left(\nabla_{E_{0}} N^{+}\right)_{0} N_{2}^{-}+\Delta N_{0}^{+}\left(\nabla_{E_{0}} N^{-}\right)_{2} \\
& +2 \mathfrak{L}_{E_{0}}\left(r^{2}\right) g_{20} \\
& =\Delta\left(\frac{a^{2} \sin \vartheta \cos \vartheta}{\rho^{2} \sqrt{|\Delta|}}\right)\left(-\frac{\rho}{\sqrt{|\Delta|}}\right)+\Delta\left(-\frac{\rho}{\sqrt{|\Delta|}}\right)\left(\frac{a^{2} \sin \vartheta \cos \vartheta}{\rho^{2} \sqrt{|\Delta|}}\right) \\
& +\Delta\left(-\frac{a^{2} \sin \vartheta \cos \vartheta}{\rho^{2} \sqrt{|\Delta|}}\right)\left(-\frac{\rho}{\sqrt{|\Delta|}}\right)+\Delta\left(-\frac{\rho}{\sqrt{|\Delta|}}\right)\left(-\frac{a^{2} \sin \vartheta \cos \vartheta}{\rho^{2} \sqrt{|\Delta|}}\right) \\
& =0 \text {, } \\
& S_{022}=\nabla_{E_{0}} K_{22}+2 \nabla_{E_{2}} K_{20} \\
& =\mathfrak{L}_{E_{0}}(\Delta) N_{2}^{+} N_{2}^{-}+\Delta\left(\nabla_{E_{0}} N^{+}\right)_{2} N_{2}^{-}+\Delta N_{2}^{+}\left(\nabla_{E_{0}} N^{-}\right)_{2}+\mathfrak{L}_{E_{0}}\left(r^{2}\right) g_{22} \\
& +\mathfrak{L}_{E_{2}}(\Delta) N_{2}^{+} N_{0}^{-}+\Delta\left(\nabla_{E_{2}} N^{+}\right)_{2} N_{0}^{-}+\Delta N_{2}^{+}\left(\nabla_{E_{2}} N^{-}\right)_{0} \\
& +\mathfrak{L}_{E_{2}}(\Delta) N_{0}^{+} N_{2}^{-}+\Delta\left(\nabla_{E_{2}} N^{+}\right)_{0} N_{2}^{-}+\Delta N_{0}^{+}\left(\nabla_{E_{2}} N^{-}\right)_{2} \\
& +2 \mathfrak{L}_{E_{2}}\left(r^{2}\right) g_{20}
\end{aligned}
$$




$$
\begin{aligned}
& =\Delta\left(\frac{r}{\rho^{2}}\right)\left(-\frac{\rho}{\sqrt{|\Delta|}}\right)+\Delta\left(-\frac{\rho}{\sqrt{|\Delta|}}\right)\left(-\frac{r}{\rho^{2}}\right) \\
& =0 \text {, } \\
& S_{300}=\nabla_{E_{3}} K_{00}+2 \nabla_{E_{0}} K_{30} \\
& =\mathfrak{L}_{E_{3}}(\Delta) N_{0}^{+} N_{0}^{-}+\Delta\left(\nabla_{E_{3}} N^{+}\right)_{0} N_{0}^{-}+\Delta N_{0}^{+}\left(\nabla_{E_{3}} N^{-}\right)_{0}+\mathfrak{L}_{E_{3}}\left(r^{2}\right) g_{00} \\
& +\mathfrak{L}_{E_{0}}(\Delta) N_{3}^{+} N_{0}^{-}+\Delta\left(\nabla_{E_{0}} N^{+}\right)_{3} N_{0}^{-}+\Delta N_{3}^{+}\left(\nabla_{E_{0}} N^{-}\right)_{0} \\
& +\mathfrak{L}_{E_{0}}(\Delta) N_{0}^{+} N_{3}^{-}+\Delta\left(\nabla_{E_{0}} N^{+}\right)_{0} N_{3}^{-}+\Delta N_{0}^{+}\left(\nabla_{E_{0}} N^{-}\right)_{3} \\
& +2 \mathfrak{L}_{E_{0}}\left(r^{2}\right) g_{30} \\
& =\Delta\left(\frac{a r \sin \vartheta}{\rho^{2} \sqrt{|\Delta|}}\right)\left(-\frac{\rho}{\sqrt{|\Delta|}}\right)+\Delta\left(-\frac{\rho}{\sqrt{|\Delta|}}\right)\left(-\frac{a r \sin \vartheta}{\rho^{2} \sqrt{|\Delta|}}\right) \\
& +\Delta\left(\frac{a r \sin \vartheta}{\rho^{2} \sqrt{|\Delta|}}\right)\left(-\frac{\rho}{\sqrt{|\Delta|}}\right)+\Delta\left(-\frac{\rho}{\sqrt{|\Delta|}}\right)\left(-\frac{a r \sin \vartheta}{\rho^{2} \sqrt{|\Delta|}}\right) \\
& =0 \text {, } \\
& S_{033}=\nabla_{E_{0}} K_{33}+2 \nabla_{E_{3}} K_{30} \\
& =\mathfrak{L}_{E_{0}}(\Delta) N_{3}^{+} N_{3}^{-}+\Delta\left(\nabla_{E_{0}} N^{+}\right)_{3} N_{3}^{-}+\Delta N_{3}^{+}\left(\nabla_{E_{0}} N^{-}\right)_{3}+\mathfrak{L}_{E_{0}}\left(r^{2}\right) g_{33} \\
& +\mathfrak{L}_{E_{3}}(\Delta) N_{3}^{+} N_{0}^{-}+\Delta\left(\nabla_{E_{3}} N^{+}\right)_{3} N_{0}^{-}+\Delta N_{3}^{+}\left(\nabla_{E_{3}} N^{-}\right)_{0} \\
& +\mathfrak{L}_{E_{3}}(\Delta) N_{0}^{+} N_{3}^{-}+\Delta\left(\nabla_{E_{3}} N^{+}\right)_{0} N_{3}^{-}+\Delta N_{0}^{+}\left(\nabla_{E_{3}} N^{-}\right)_{3} \\
& +2 \mathfrak{L}_{E_{3}}\left(r^{2}\right) g_{30} \\
& =\Delta\left(\frac{r}{\rho^{2}}\right)\left(-\frac{\rho}{\sqrt{|\Delta|}}\right)+\Delta\left(-\frac{\rho}{\sqrt{|\Delta|}}\right)\left(-\frac{r}{\rho^{2}}\right) \\
& =0 \text {. } \\
& 2 S_{123}=2 \nabla_{E_{1}} K_{23}+2 \nabla_{E_{2}} K_{13}+2 \nabla_{E_{3}} K_{12} \\
& =\mathfrak{L}_{E_{1}}(\Delta)\left(N_{2}^{+} N_{3}^{-}+N_{3}^{+} N_{2}^{-}\right) \\
& +\Delta\left(\nabla_{E_{1}} N^{+}\right)_{2} N_{3}^{-}+\Delta N_{2}^{+}\left(\nabla_{E_{1}} N^{-}\right)_{3} \\
& +\Delta\left(\nabla_{E_{1}} N^{+}\right)_{3} N_{2}^{-}+\Delta N_{3}^{+}\left(\nabla_{E_{1}} N^{-}\right)_{2}+2 \mathfrak{L}_{E_{1}}\left(r^{2}\right) g_{23} \\
& +\mathfrak{\sim}_{E_{2}}(\Delta)\left(N_{1}^{+} V_{3}^{-}+N_{3}^{+} N_{1}^{-}\right) \\
& +\Delta\left(\nabla_{E_{2}} N^{+}\right)_{1} V_{3}^{-}+\Delta N_{1}^{+}\left(\nabla_{E_{2}} N^{-}\right)_{3} \\
& +\Delta\left(\nabla_{E_{2}} N^{+}\right)_{3} N_{1}^{-}+\Delta N_{3}^{+}\left(\nabla_{E_{2}} N^{-}\right)_{1}+2 \mathfrak{L}_{E_{2}}\left(r^{2}\right) g_{13} \\
& +\mathfrak{L}_{E_{3}}(\Delta)\left(N_{1}^{+} N_{2}^{-}+N_{2}^{+} N_{1}^{-}\right) \\
& +\Delta\left(\nabla_{E_{3}} N^{+}\right)_{1} N_{2}^{-}+\Delta N_{1}^{+}\left(\nabla_{E_{3}} N^{-}\right)_{2} \\
& +\Delta\left(\nabla_{E_{3}} N^{+}\right)_{2} N_{1}^{-}+\Delta N_{2}^{+}\left(\nabla_{E_{3}} N^{-}\right)_{1}+2 \mathfrak{L}_{E_{3}}\left(r^{2}\right) g_{12}
\end{aligned}
$$




$$
\begin{aligned}
& =\Delta\left(\epsilon \frac{\rho}{\sqrt{|\Delta|}}\right)\left(\frac{a \cos \vartheta}{\rho^{2}}\right)+\Delta\left(\frac{a \cos \vartheta}{\rho^{2}}\right)\left(-\epsilon \frac{\rho}{\sqrt{|\Delta|}}\right) \\
& +\Delta\left(\epsilon \frac{\rho}{\sqrt{|\Delta|}}\right)\left(-\frac{a \cos \vartheta}{\rho^{2}}\right)+\Delta\left(-\frac{a \cos \vartheta}{\rho^{2}}\right)\left(-\epsilon \frac{\rho}{\sqrt{|\Delta|}}\right) \\
& =0 \text {, } \\
& 2 S_{023}=2 \nabla_{E_{0}} K_{23}+2 \nabla_{E_{2}} K_{03}+2 \nabla_{E_{3}} K_{02} \\
& =\mathfrak{L}_{E_{0}}(\Delta)\left(N_{2}^{+} N_{3}^{-}+V_{3}^{+} N_{2}^{-}\right) \\
& +\Delta\left(\nabla_{E_{0}} N^{+}\right)_{2} N_{3}^{-}+\Delta N_{2}^{+}\left(\nabla_{E_{0}} N^{-}\right)_{3} \\
& +\Delta\left(\nabla_{E_{0}} N^{+}\right)_{3} N_{2}^{-}+\Delta N_{3}^{+}\left(\nabla_{E_{0}} N^{-}\right)_{2}+2 \mathfrak{L}_{E_{0}}\left(r^{2}\right) g_{23} \\
& +\mathfrak{L}_{E_{2}}(\Delta)\left(N_{0}^{+} N_{3}^{-}+N_{3}^{+} N_{0}^{-}\right) \\
& +\Delta\left(\nabla_{E_{2}} N^{+}\right)_{0} \nu_{3}^{-}+\Delta N_{0}^{+}\left(\nabla_{E_{2}} N^{-}\right)_{3} \\
& +\Delta\left(\nabla_{E_{2}} N^{+}\right)_{3} V_{0}^{-}+\Delta N_{3}^{+}\left(\nabla_{E_{2}} N^{-}\right)_{0}+2 \mathfrak{L}_{E_{2}}\left(r^{2}\right) g_{03} \\
& +\mathfrak{L}_{E_{3}}(\Delta)\left(N_{0}^{+} N_{2}^{-}+N_{2}^{+} N_{0}^{-}\right) \\
& +\Delta\left(\nabla_{E_{3}} N^{+}\right)_{0} N_{2}^{-}+\Delta N_{0}^{+}\left(\nabla_{E_{3}} N^{-}\right)_{2} \\
& +\Delta\left(\nabla_{E_{3}} N^{+}\right)_{2} N_{0}^{-}+\Delta N_{2}^{+}\left(\nabla_{E_{3}} N^{-}\right)_{0}+2 \mathfrak{L}_{E_{3}}\left(r^{2}\right) g_{02} \\
& =\Delta\left(-\frac{\rho}{\sqrt{|\Delta|}}\right)\left(\frac{a \cos \vartheta}{\rho^{2}}\right)+\Delta\left(\frac{a \cos \vartheta}{\rho^{2}}\right)\left(-\frac{\rho}{\sqrt{|\Delta|}}\right) \\
& +\Delta\left(-\frac{\rho}{\sqrt{|\Delta|}}\right)\left(-\frac{a \cos \vartheta}{\rho^{2}}\right)+\Delta\left(-\frac{a \cos \vartheta}{\rho^{2}}\right)\left(-\frac{\rho}{\sqrt{|\Delta|}}\right) \\
& =0 \text {, } \\
& 2 S_{103}=2 \nabla_{E_{1}} K_{03}+2 \nabla_{E_{0}} K_{13}+2 \nabla_{E_{3}} K_{10} \\
& =\mathfrak{L}_{E_{1}}(\Delta)\left(N_{0}^{+} N_{3}^{-}+N_{3}^{+} N_{0}^{-}\right) \\
& +\Delta\left(\nabla_{E_{1}} N^{+}\right)_{0} N_{3}^{-}+\Delta N_{0}^{+}\left(\nabla_{E_{1}} N^{-}\right)_{3} \\
& +\Delta\left(\nabla_{E_{1}} N^{+}\right)_{3} V_{0}^{-}+\Delta N_{3}^{+}\left(\nabla_{E_{1}} N^{-}\right)_{0}+2 \mathfrak{L}_{E_{1}}\left(r^{2}\right) g_{03} \\
& +\mathfrak{L}_{E_{0}}(\Delta)\left(N_{1}^{+} N_{3}^{-}+N_{3}^{+} N_{1}^{-}\right) \\
& +\Delta\left(\nabla_{E_{0}} N^{+}\right)_{1} N_{3}^{-}+\Delta N_{1}^{+}\left(\nabla_{E_{0}} N^{-}\right)_{3} \\
& +\Delta\left(\nabla_{E_{0}} N^{+}\right)_{3} N_{1}^{-}+\Delta N_{3}^{+}\left(\nabla_{E_{0}} N^{-}\right)_{1}+2 \mathfrak{L}_{E_{0}}\left(r^{2}\right) g_{13} \\
& +\mathfrak{L}_{E_{3}}(\Delta)\left(N_{1}^{+} N_{0}^{-}+N_{0}^{+} N_{1}^{-}\right) \\
& +\Delta\left(\nabla_{E_{3}} N^{+}\right)_{1} v_{0}^{-}+\Delta N_{1}^{+}\left(\nabla_{E_{3}} N^{-}\right)_{0} \\
& +\Delta\left(\nabla_{E_{3}} N^{+}\right)_{0} \cdot V_{1}^{-}+\Delta N_{0}^{+}\left(\nabla_{E_{3}} N^{-}\right)_{1}+2 \mathfrak{L}_{E_{3}}\left(r^{2}\right) g_{10}
\end{aligned}
$$




$$
\begin{aligned}
& =\Delta\left(-\frac{\rho}{\sqrt{|\Delta|}}\right)\left(-\epsilon \frac{\operatorname{ar} \sin \vartheta}{\rho^{2} \sqrt{|\Delta|}}\right)+\Delta\left(-\epsilon \frac{a r \sin \vartheta}{\rho^{2} \sqrt{|\Delta|}}\right)\left(-\frac{\rho}{\sqrt{|\Delta|}}\right) \\
& +\Delta\left(\epsilon \frac{\rho}{\sqrt{|\Delta|}}\right)\left(-\frac{a r \sin \vartheta}{\rho^{2} \sqrt{|\Delta|}}\right)+\Delta\left(\frac{a r \sin \vartheta}{\rho^{2} \sqrt{|\Delta|}}\right)\left(-\epsilon \frac{\rho}{\sqrt{|\Delta|}}\right) \\
& +\Delta\left(-\epsilon \frac{\operatorname{ar} \sin \vartheta}{\rho^{2} \sqrt{|\Delta|}}\right)\left(-\frac{\rho}{\sqrt{|\Delta|}}\right)+\Delta\left(\epsilon \frac{\rho}{\sqrt{|\Delta|}}\right)\left(-\frac{a r \sin \vartheta}{\rho^{2} \sqrt{|\Delta|}}\right) \\
& +\Delta\left(\frac{a r \sin \vartheta}{\rho^{2} \sqrt{|\Delta|}}\right)\left(-\epsilon \frac{\rho}{\sqrt{|\Delta|}}\right)+\Delta\left(-\frac{\rho}{\sqrt{|\Delta|}}\right)\left(-\epsilon \frac{\operatorname{ar} \sin \vartheta}{\rho^{2} \sqrt{|\Delta|}}\right) \\
& =0 \text {, } \\
& 2 S_{120}=2 \nabla_{E_{1}} K_{20}+2 \nabla_{E_{2}} K_{10}+2 \nabla_{E_{0}} K_{12} \\
& =\mathfrak{L}_{E_{1}}(\Delta)\left(N_{2}^{+} N_{0}^{-}+N_{0}^{+} N_{2}^{-}\right) \\
& +\Delta\left(\nabla_{E_{1}} N^{+}\right)_{2} N_{0}^{-}+\Delta N_{2}^{+}\left(\nabla_{E_{1}} N^{-}\right)_{0} \\
& +\Delta\left(\nabla_{E_{1}} N^{+}\right)_{0} N_{2}^{-}+\Delta N_{0}^{+}\left(\nabla_{E_{1}} N^{-}\right)_{2}+2 \mathfrak{L}_{E_{1}}\left(r^{2}\right) g_{20} \\
& +\mathfrak{\mathfrak { L }}_{E_{2}}(\Delta)\left(N_{1}^{+} \cdot N_{0}^{-}+N_{0}^{+} N_{1}^{-}\right) \\
& +\Delta\left(\nabla_{E_{2}} N^{+}\right)_{1} N_{0}^{-}+\Delta N_{1}^{+}\left(\nabla_{E_{2}} N^{-}\right)_{0} \\
& +\Delta\left(\nabla_{E_{2}} N^{+}\right)_{0} N_{1}^{-}+\Delta N_{0}^{+}\left(\nabla_{E_{2}} N^{-}\right)_{1}+2 \mathfrak{L}_{E_{2}}\left(r^{2}\right) g_{10} \\
& +\mathfrak{L}_{E_{0}}(\Delta)\left(N_{1}^{+} V_{2}^{-}+N_{2}^{+} N_{1}^{-}\right) \\
& +\Delta\left(\nabla_{E_{0}} N^{+}\right)_{1} N_{2}^{-}+\Delta N_{1}^{+}\left(\nabla_{E_{0}} N^{-}\right)_{2} \\
& +\Delta\left(\nabla_{E_{0}} N^{+}\right)_{2} N_{1}^{-}+\Delta N_{2}^{+}\left(\nabla_{E_{0}} N^{-}\right)_{1}+2 \mathfrak{L}_{E_{0}}\left(r^{2}\right) g_{12} \\
& =\Delta\left(\epsilon \frac{a^{2} \sin \vartheta \cos \vartheta}{\rho^{2} \sqrt{|\Delta|}}\right)\left(-\frac{\rho}{\sqrt{|\Delta|}}\right)+\Delta\left(-\frac{\rho}{\sqrt{|\Delta|}}\right)\left(-\epsilon \frac{a^{2} \sin \vartheta \cos \vartheta}{\rho^{2} \sqrt{|\Delta|}}\right) \\
& +\Delta\left(-\epsilon \frac{a^{2} \sin \vartheta \cos \vartheta}{\rho^{2} \sqrt{|\Delta|}}\right)\left(-\frac{\rho}{\sqrt{|\Delta|}}\right)+\Delta\left(\epsilon \frac{\rho}{\sqrt{|\Delta|}}\right)\left(\frac{a^{2} \sin \vartheta \cos \vartheta}{\rho^{2} \sqrt{|\Delta|}}\right) \\
& +\Delta\left(\frac{a^{2} \sin \vartheta \cos \vartheta}{\rho^{2} \sqrt{|\Delta|}}\right)\left(-\epsilon \frac{\rho}{\sqrt{|\Delta|}}\right)+\Delta\left(-\frac{\rho}{\sqrt{|\Delta|}}\right)\left(\epsilon \frac{a^{2} \sin \vartheta \cos \vartheta}{\rho^{2} \sqrt{|\Delta|}}\right) \\
& +\Delta\left(\epsilon \frac{\rho}{\sqrt{|\Delta|}}\right)\left(-\frac{a^{2} \sin \vartheta \cos \vartheta}{\rho^{2} \sqrt{|\Delta|}}\right)+\Delta\left(-\frac{a^{2} \sin \vartheta \cos \vartheta}{\rho^{2} \sqrt{|\Delta|}}\right)\left(-\epsilon \frac{\rho}{\sqrt{|\Delta|}}\right) \\
& =0 \text {. }
\end{aligned}
$$




\section{Bibliografia}

[1] R. Abraham, J.E. Marsden: Foundations of Mechanics, Addison Wesley, New York 1987.

[2] B. Carter: Global Structure of the Kerr Family of Gravitational Fields, Phys. Rev. 174 (1968) 15.59-1571.

[3] B. Carter: Hamilton-Jacobi and Schrödinger Separable Solution of Einstein's Equations, Commun. Math. Phys. 10 (1968) 280-310.

[4] B. Carter: Killing Tensor Quantum Numbers and Conserved Currents in Curved Space, Phys. Rev. D 16 (1977) 3395.

[5] M. Dubois-Violette, P.W. Michor: A Common Generalization of the Frölicher-Nijenhuis Bracket and the Schouten Bracket for Symmetric Multivector Fields, Indag. Math., N.S., 6 (1995) 51-66.

[6] A.T. Fomenko, V.V. Trofimov: Integrable Systems on Lie Algebras and Symmetric Spaces, Gordon and Breach, New York 1988.

[7] G.W Gibbons, R.H. Rietdijk, J.W. van Holten: SUZY in the Sky, Nucl. Phys. B 404 (1993) 42-64.

[8] S.W. Hawking, G.F.R. Ellis: The Large Scale Structure of Space-Time, Cambridge University Press. Cambridge 1973.

[9] R.P. Kerr: Gravitational Field of a Spinning Mass as an Example of Algebraically Special Metric, Phys. Rev. Lett. 11 (1963) 237-238.

[10] V.V. Kozlov: Symmetries. Topology and Resonances in Hamiltonian Mechanics, Springer Verlag, Berlin 1991.

[11] C.W. Misner, K.S. Thorne, J.A. Wheeler: Gravitation, Freeman \& Co., San Francisco 1973.

[12] B. O'Neill: The Geometry of Kerr Black Holes. A.K. Peters, Wellesley 1995. 
[13] G.E. Prince, M. Crampin: Projetive Differential Geometry and Geodesic Conservation Law in General Relativity. I: Projective Actions, Gen. Rel. Grav. 16 (1984) 921-942.

[14] G.E. Prince, M. Crampin: Projetive Differential Geometry and Geodesic Conservation Law in General Relativity. II: Conservation Laws, Gen. Rel. Grav. 16 (1984) 1063-1075.

[15] R.H. Rietdijk, J.W. van Holten: Killing Tensors and a New Duality, Nucl. Phys. B 472 (1996) $427-446$.

[16] K. Rosquist: Killing Tensor Conservation Laws and Their Generators, J. Math. Phys. 30 (1989) 2319-2321.

[17] K. Rosquist, M. Goliath: Lax Pair Tensors and Integrable Spacetimes, Gen. Rel. Grav. 30 (1998) 921-942.

[18] J.A. Schouten: ̈̈ber Differentialkonkomitanten zweier kontravarianter Grössen, Indag. Math., N.S., 22 (1940) 937-942.

[19] R. Wald: General Relativity, Chicago University Press, Chicago 1984.

[20] M. Walker, R. Penrose: On Quadratic First Integrals of the Geodesic Equations for Type $\{22\}$ Spacetimes, Commun. Math. Phys. 18 (1970) 265-274.

[21] S.N. Zhang, W. Cui, W. Chen: Black Holes Spin in X-Ray Binaries: Observational Consequences, Astrophys. J. 482 (1997) L155-L158.

[22] S.N. Zhang, W. Cui, W. Chen: Evidence for Frame-Dragging Around Spinning Black Holes in X-Ray Binaries, Astrophys. J. 492 (1998) L153-L157. 